Pacific Journal of Mathematics

THE SELBERG TRACE FORMULA. VII. APPLICATION OF
THE TRUNCATION PROCESS TO THE CONTINUOUS
SPECTRUM

M. SCOTt Osborne AND GARTH WilLiam Warner, JR. 


\section{THE SELBERG TRACE FORMULA VII: APPLICATION OF THE TRUNCATION PROCESS TO THE CONTINUOUS SPECTRUM}

\section{Scott Osborne AND GARTh WARner}

This is the seventh in a projected series of papers in which we plan to come to grips with the Selberg trace formula, the ultimate objective being a reasonably explicit expression. As the title suggests, our goal in this work is to prepare the stage for the complete delineation of the contribution to the trace coming from the continuous spectrum, concentrating here on the role played by the truncation process. In our next publication, we shall finish the investigation, setting down the precise form of the various terms in all detail.

1. Introduction. Supposing that the pair $(G, \Gamma)$ is as usual, the starting point for all this is the formula for

$$
\operatorname{tr}\left(Q^{\mathbf{H}} \circ L_{G / \Gamma}^{\text {dis }}(\alpha) \circ Q^{\mathbf{H}}\right)
$$

given in $\S 8$ of [2-(f)]. Thus, let $\alpha$ be a $K$-central, $K$-finite element of $C_{c}^{\infty}(G)$-then $\bmod o(\mathbf{H})$

$$
\operatorname{tr}\left(Q^{\mathbf{H}} \circ L_{G / \Gamma}^{\mathrm{dis}}(\alpha) \circ Q^{\mathbf{H}}\right)
$$

is the same as

$$
\mathbf{K}\left(\mathbf{H}_{O}: \alpha: \Gamma\right)
$$

less

$$
\begin{aligned}
& \sum_{i=1}^{r-1}(-1)^{\operatorname{rank}\left(P_{\imath}\right)} p\left(\Gamma: P_{i}: \mathbf{H}\left(P_{i}\right)-\mathbf{H}_{\mathrm{O}}\left(P_{i}\right)\right) \\
& \cdot \operatorname{tr}\left(Q^{I_{M_{i}}(\mathbf{H})} \circ L_{M_{i} / \Gamma_{M_{l}}}^{\mathrm{dis}}\left(\alpha^{P_{\imath}}\right) \circ Q^{I_{M_{\imath}}(\mathbf{H})}\right)
\end{aligned}
$$

less

$$
\begin{gathered}
\sum_{i=1}^{r}(-1)^{\operatorname{rank}\left(P_{i}\right)} p\left(\Gamma: P_{i}: \mathbf{H}\left(P_{i}\right)-\mathbf{H}_{\mathrm{O}}\left(P_{i}\right)\right) \\
\cdot \operatorname{tr}\left(Q^{I_{M_{i}}(\mathbf{H})} \circ L_{M_{i} / \Gamma_{M_{i}}}^{\operatorname{con}}\left(\alpha^{P_{i}}\right) \circ Q^{I_{M_{i}}(\mathbf{H})}\right),
\end{gathered}
$$

the sums running over a set of representatives for the $\Gamma$-conjugacy classes of $\Gamma$-cuspidal split parabolic subgroups of $G$, the first of these 
being proper ( $r$ is the index corresponding to $G$ itself). In this connection, recall that according to our conventions (cf. [2-(f), §5]), o(H) stands for a function on $\mathfrak{a}_{Q}$ such that

$$
\lim _{\mathbf{H} \rightarrow-\infty} \mathrm{o}(\mathbf{H})=0 \text {. }
$$

It will not be necessary to spell out the definition of

$$
\mathbf{K}\left(\mathbf{H}_{\mathrm{O}}: \alpha: \Gamma\right)
$$

since it contains the contribution to the trace associated with the conjugacy classes, a topic that will be dealt with elsewhere. As for the other terms, note that they involve both the discrete spectrum and the continuous spectrum at all lower levels but that the continuous spectrum is present at even the top level. Because

$$
p\left(\Gamma: P_{i}: ?\right)
$$

is an Arthur polynomial (cf. [2-(f), §7]), hence possesses certain remarkable combinatorial properties, it is tempting to suspect that a great deal of cancellation should take place. This, in fact, is exactly what happens although it will become fully apparent only later on.

To anticipate the eventual outcome, let us abbreviate by writing

$$
\begin{aligned}
\operatorname{tr}\left(Q^{\mathbf{H}} \circ L_{G / \Gamma}^{\text {dis }}(\alpha) \circ Q^{\mathbf{H}}\right)= & \mathbf{K}\left(\mathbf{H}_{\mathrm{O}}: \alpha: \Gamma\right) \\
& +\mathbf{F n c}\left(\mathbf{H}: \mathbf{H}_{\mathrm{O}}: \alpha: \Gamma\right)+\mathbf{o}(\mathbf{H}) .
\end{aligned}
$$

Then the central idea of the cancellation is to show that

$$
\operatorname{Fnc}\left(\mathbf{H}: \mathbf{H}_{\mathrm{O}}: \alpha: \Gamma\right)=\operatorname{Fnc}\left(\mathbf{H}_{\mathrm{O}}: \alpha: \Gamma\right)+\operatorname{Con}-\mathbf{S p}(\alpha: \Gamma)+\mathbf{o}(\mathbf{H}),
$$

the upshot being that Con $-\mathbf{S p}(\alpha: \Gamma)$ is independent of $\mathbf{H}$ and $\mathbf{H}_{\mathrm{O}}$, thus represents the contribution to the trace furnished by the continuous spectrum.

Turning to the organization of this article, certain preliminary facts are collected in $\S 2$, while $\S \S 3-4$ have as their theme various results from the theory of Arthur polynomials and Detroit families. These are then applied in $\S 5$ and $\S 7$ to the leading term of two truncated Eisenstein series (be they complete or partial), $\S 6$ representing a technical but necessary intermediary. In $\S 8$, we recast into the boldface picture the formula for

$$
\operatorname{tr}\left(Q^{\mathbf{H}} \circ L_{G / \Gamma}^{\mathrm{dis}}(\alpha) \circ Q^{\mathbf{H}}\right)
$$

mentioned above, thereby paving the way for the integral manipulations in $\S 9$, leading finally to the main result of the paper, namely 
Theorem 9.7. To see its potential implications, two examples are given in $\S 10$.

As a general reference and suggested overall introduction to the subject, we shall use our monograph:

The Theory of Eisenstein Systems, Academic Press, N.Y., 1981.

Throughout the sequel, the title of this work has been abbreviated to TES.

2. The kernel of the continuous spectrum. The purpose of this section is to set down some fundamental facts which constitute essential background for all that follows.

Let $G$ be a reductive Lie group, $\Gamma$ a lattice in $G$, both subject to the usual conditions. Agreeing to employ standard notation (see, in particular, $\S 2$ of [2-(f)]), let $\alpha$ be a $K$-finite element of $C_{c}^{\infty}(G)$-then $L_{G / \Gamma}^{\text {con }}(\alpha)$, the restriction of $L_{G / \Gamma}(\alpha)$ to $L_{\text {con }}^{2}(G / \Gamma)$, is an integral operator admitting a kernel

$$
K_{\alpha}^{\mathrm{con}}(x, y) \in S_{r}^{\infty}(G / \Gamma \times G / \Gamma),
$$

where $r$ is independent of $\alpha$. Furthermore, in view of Proposition 2.3 of [2-(f)], $\forall \mathbf{H} \in \mathfrak{a}_{Q}$, the truncation

$$
Q^{\mathbf{H}} \circ L_{G / \Gamma}^{\text {con }}(\alpha) \circ Q^{\mathbf{H}}
$$

is of the trace class, its trace

$$
\operatorname{tr}\left(Q^{\mathbf{H}} \circ L_{G / \Gamma}^{\operatorname{con}}(\alpha) \circ Q^{\mathbf{H}}\right)
$$

being the integral

$$
\int_{G / \Gamma} Q_{(1)}^{\mathbf{H}} Q_{(2)}^{\mathbf{H}} K_{\alpha}^{\mathrm{con}}(x, x) d_{G}(x) .
$$

The determination of $K_{\alpha}^{\text {con }}$ in terms of Eisenstein series may be found in Chapter 8 of TES. As it will be needed, we had best recall it. Fix an association class $\mathscr{C} \neq\{G\}$ of $\Gamma$-cuspidal split parabolic subgroups of $G$. Let $P_{i}=M_{i} \cdot A_{i} \cdot N_{i}(1 \leq i \leq r)$ be a set of representatives for $G \backslash \mathscr{C}$. Let $\mathscr{\theta}=\left\{\boldsymbol{\theta}_{i}: i=1, \ldots, r\right\}$ be an orbit type-then the representation space $\mathscr{E}\left(\boldsymbol{\theta}_{i}\right)$ for

$$
\operatorname{Ind}_{M_{i} \cdot A_{i} \cdot N_{l}}^{G}\left(\left(\boldsymbol{\theta}_{i}, \Lambda_{i}\right)\right)
$$

is the orthogonal direct sum over $\delta \in \hat{K}$ of the corresponding isotypic components $\mathscr{E}\left(\delta, \boldsymbol{\theta}_{i}\right)$. Let $\left\{\mathbf{e}_{n}^{i}: n \in I\left(\boldsymbol{\theta}_{i}\right)\right\}$ be an orthonormal basis for $\mathscr{E}\left(\boldsymbol{\sigma}_{i}\right)$ so selected that each $\mathrm{e}_{n}^{i}$ lies in some $\mathscr{E}\left(\delta, \boldsymbol{\theta}_{i}\right)$. Set

$$
\left.\mathbf{C}_{m n}\left(\alpha: \boldsymbol{\theta}_{i}, \Lambda_{i}\right)=\left(\operatorname{Ind}_{M_{i} \cdot A_{i} \cdot N_{l}}^{G}\left(\boldsymbol{\sigma}_{i}, \Lambda_{i}\right)\right)(\alpha) \mathbf{e}_{n}^{i}, \mathbf{e}_{m}^{i}\right) .
$$


Form

$$
\begin{aligned}
& K_{\alpha}\left(x, y: \boldsymbol{\theta}_{i}, \Lambda_{i}\right) \\
& \quad=\sum_{m, n} C_{m n}\left(\alpha: \boldsymbol{\theta}_{i}, \Lambda_{i}\right) \cdot \mathbf{E}\left(P_{i} \mid A_{i}: \mathbf{e}_{m}^{i}: \Lambda_{i}: x\right) \overline{\mathbf{E}\left(P_{i} \mid A_{i}: \mathbf{e}_{n}^{i}: \Lambda_{i}: y\right)},
\end{aligned}
$$

$\mathbf{E}\left(P_{i} \mid A_{i}: \ldots\right)$ an Eisenstein series. Write

$$
K_{\alpha}(x, y: \mathscr{\theta} ; \mathscr{C})
$$

in place of

$$
\frac{1}{(2 \pi)^{l}} \cdot \frac{1}{*(\mathscr{C})} \cdot \sum_{i=1}^{r} \int_{\operatorname{Re}\left(\Lambda_{l}\right)=0} K_{\alpha}\left(x, y: \boldsymbol{\sigma}_{i}, \Lambda_{i}\right)\left|d \Lambda_{i}\right|,
$$

and then put

$$
K_{\alpha}(x, y: \mathscr{C})=\sum_{\mathscr{\theta}} K_{\alpha}(x, y: \mathscr{\theta} ; \mathscr{C}) .
$$

Here, $l$ is the rank of $\mathscr{C}$, while $*(\mathscr{C})$ is the cardinality of the set of chambers in a split component of any member of $\mathscr{C}$.

With this notation, the kernel $K_{\alpha}^{\text {con }}$ of $L_{G / \Gamma}^{\text {con }}(\alpha)$ is

$$
\sum_{\mathscr{C}} K_{\alpha}(x, y: \mathscr{C})
$$

the slash meaning that the sum is over the $\mathscr{C} \neq\{G\}$.

LEMMA 2.1. There exist real numbers $r^{\prime}, r^{\prime \prime}$ such that for all Siegel domains $\mathfrak{S}^{\prime}, \mathfrak{S}^{\prime \prime}$ associated with $\Gamma$-percuspidals $P^{\prime}, P^{\prime \prime}$ there is a positive constant $C$ such that

$$
\sum_{\boldsymbol{\theta}} \sum_{i=1}^{r} \int_{\operatorname{Re}\left(\Lambda_{i}\right)=0}\left|K_{\alpha}\left(x, y: \boldsymbol{\theta}_{i}, \Lambda_{i}\right)\right|\left|d \Lambda_{i}\right| \leq C \cdot \Xi_{P^{\prime}}(x)^{r^{\prime}} \Xi_{P^{\prime \prime}}(y)^{r^{\prime \prime}}
$$

for every $x \in \mathfrak{S}^{\prime}, y \in \mathfrak{S}^{\prime \prime}$.

[This is Lemma 8.4 in TES. Incidentally, it can be shown that $r^{\prime}, r^{\prime \prime}$ can be taken independent of $\alpha$ (cf. p. 365 of TES).]

Passing to constant terms will have no effect on the estimate, so

$$
K_{\alpha}\left(x, y: \boldsymbol{\theta}_{i}, \Lambda_{i}\right)
$$

can be exchanged for

$$
Q_{(1)}^{\mathbf{H}} Q_{(2)}^{\mathbf{H}} K_{\alpha}\left(x, y: \boldsymbol{\sigma}_{i}, \Lambda_{i}\right) .
$$


Since the constant term integrals are compactly supported, they can be iterated leading, therefore, to the conclusion that

$$
Q_{(1)}^{\mathbf{H}} Q_{(2)}^{\mathbf{H}} K_{\alpha}^{\mathrm{con}}(x, y)
$$

is equal to

$$
\begin{aligned}
& \sum_{\mathscr{C}} \frac{1}{(2 \pi)^{l}} \cdot \frac{1}{*(\mathscr{C})} \cdot \sum_{\mathscr{O}} \sum_{i=1}^{r} \\
& \quad \times \int_{\operatorname{Re}\left(\Lambda_{i}\right)=0} Q_{(1)}^{\mathbf{H}} Q_{(2)}^{\mathbf{H}} K_{\alpha}\left(x, y: \boldsymbol{\theta}_{i}, \Lambda_{i}\right)\left|d \Lambda_{i}\right| .
\end{aligned}
$$

To explicate

$$
\operatorname{tr}\left(Q^{\mathbf{H}} \circ L_{G / \Gamma}^{\operatorname{con}}(\alpha) \circ Q^{\mathbf{H}}\right)
$$

or still

$$
\int_{G / \Gamma} Q_{(1)}^{\mathbf{H}} Q_{(2)}^{\mathbf{H}} K_{\alpha}^{\mathrm{con}}(x, x) d_{G}(x),
$$

start by inserting

$$
\sum_{\mathscr{C}} \cdots \int_{\operatorname{Re}\left(\Lambda_{l}\right)=0} \cdots
$$

The problem now is to push $\int_{G / \Gamma}$ all the way to the right past the $\sum-\int$ signs.

LEMMA 2.2. Fix $\mathscr{C} \neq\{G\}$-then

$$
\left\{\begin{array}{l}
\forall \Gamma \text {-percuspidal } P_{0}, \\
\forall \text { Siegel domain } \mathfrak{S}_{0} \text { associated with } P_{0}, \\
\forall \text { real } r
\end{array}\right.
$$

$\exists C>0$ such that

$$
\sum_{\boldsymbol{\theta}} \sum_{i=1}^{r} \int_{\operatorname{Re}\left(\Lambda_{\imath}\right)=0}\left|Q_{(1)}^{\mathbf{H}} Q_{(2)}^{\mathbf{H}} K_{\alpha}\left(x, x: \boldsymbol{\theta}_{i}, \Lambda_{i}\right)\right|\left|d \Lambda_{l}\right| \leq C \cdot \Xi_{P_{0}}(x)^{r}
$$

for all $x \in \mathfrak{S}_{0}$.

[We indicate an argument. Look at the proof of Lemma 8.4 in TES. Replace the $K_{\alpha}$ there by $Q_{(1)}^{\mathbf{H}} Q_{(2)}^{\mathbf{H}} K_{\alpha}$, the $\mathbf{E}$ by $Q^{\mathbf{H}} \mathbf{E}$. As the computation in the demonstration is purely formal, we eventually end up with a finite sum of nonnegative rapidly decreasing majorants of the form

$$
Q_{(1)}^{\mathbf{H}} Q_{(2)}^{\mathbf{H}} K_{\phi}^{\mathrm{con}}(x, x) \quad\left(\phi \in C_{c}^{p}(G), p \gg 0\right),
$$

from which the lemma is immediate.] 
Taking this estimate into account, the integral

$$
\int_{G / \Gamma} Q_{(1)}^{\mathbf{H}} Q_{(2)}^{\mathbf{H}} K_{\alpha}^{\mathrm{con}}(x, x) d_{G}(x)
$$

is then seen to be equal to

$$
\begin{aligned}
& \sum_{\mathscr{C}} \frac{1}{(2 \pi)^{l}} \cdot \frac{1}{*(\mathscr{C})} \cdot \sum_{\mathscr{\theta}} \sum_{i=1}^{r} \\
& \quad \times \int_{\operatorname{Re}\left(\Lambda_{i}\right)=0}\left(\int_{G / \Gamma} Q_{(1)}^{\mathbf{H}} Q_{(2)}^{\mathbf{H}} K_{\alpha}\left(x, x: \boldsymbol{\theta}_{i}, \Lambda_{i}\right) d_{G}(x)\right)\left|d \Lambda_{i}\right|,
\end{aligned}
$$

it being a question throughout of absolute convergence. Because the sum defining

$$
K_{\alpha}\left(x, x: \boldsymbol{\sigma}_{i}, \Lambda_{i}\right)
$$

is finite,

$$
\begin{aligned}
\int_{G / \Gamma} & Q_{(1)}^{\mathbf{H}} Q_{(2)}^{\mathbf{H}} K_{\alpha}\left(x, x: \boldsymbol{\sigma}_{i}, \Lambda_{i}\right) d_{G}(x) \\
= & \sum_{m, n} C_{m n}\left(\alpha: \boldsymbol{\sigma}_{i}, \Lambda_{i}\right) \\
& \cdot \int_{G / \Gamma} Q^{\mathbf{H}} \mathbf{E}\left(P_{i} \mid A_{i}: \mathbf{e}_{m}^{i}: \Lambda_{i}: x\right) Q^{\mathbf{H}} \overline{\mathbf{E}\left(P_{i} \mid A_{i}: \mathbf{e}_{n}^{i}: \Lambda_{i}: x\right)} d_{G}(x) .
\end{aligned}
$$

The inner products

$$
\left(Q^{\mathbf{H}} \mathbf{E}\left(P_{i} \mid A_{i}: \mathbf{e}_{m}^{i}: \Lambda_{i}: ?\right), Q^{\mathbf{H}} \mathbf{E}\left(P_{i} \mid A_{i}: \mathbf{e}_{n}^{i}: \Lambda_{i}: ?\right)\right)
$$

fall under the purview of the theory developed by us in [2-(b)], [2-(c)], and [2-(d)]. Before we summarize and expand upon what has been said there, some preparation is in order; cf. infra.

To recapitulate:

Proposition 2.3. Let $\alpha \in C_{c}^{\infty}(G)$ be $K$-finite-then $\forall \mathbf{H} \in \mathfrak{a}_{Q}$,

$$
\operatorname{tr}\left(Q^{\mathbf{H}} \circ L_{G / \Gamma}^{\operatorname{con}}(\alpha) \circ Q^{\mathbf{H}}\right)
$$

is equal to

$$
\begin{aligned}
\sum_{\mathscr{C}} & \frac{1}{(2 \pi)^{l}} \cdot \frac{1}{*(\mathscr{C})} \cdot \sum_{\mathscr{Q}} \sum_{i=1}^{r} \\
& \times \int_{\operatorname{Re}\left(\Lambda_{i}\right)=0} \sum_{m, n} \mathbf{C}_{m n}\left(\alpha: \boldsymbol{\theta}_{i} \cdot \Lambda_{i}\right) \\
& \cdot\left(Q^{\mathbf{H}} \mathbf{E}\left(P_{i} \mid A_{i}: \mathbf{e}_{m}^{i}: \Lambda_{i}: ?\right), Q^{\mathbf{H}} \mathbf{E}\left(P_{i} \mid A_{i}: \mathbf{e}_{n}^{i}: \Lambda_{i}: ?\right)\right)\left|d \Lambda_{i}\right|
\end{aligned}
$$


3. Addition and multiplication. The purpose of this section is to develop certain addition and multiplication rules for the $\Gamma$-functions (cf. $\S 2$ of [2-(d)]) and the Arthur polynomials (cf. $\S 7$ of [2-(f)]). These results will play a technical but important role in the sequel.

Let us place ourselves in the setting of $\S 2$ of [2-(d)], the data thus being

(1) A finite dimensional inner product space $(V,(?, ?))$ of dimension $l$, say;

(2) A basis $\left\{\lambda_{1}, \ldots, \lambda_{l}\right\}$ of $V$ subject to the condition

$$
\left(\lambda_{i}, \lambda_{j}\right) \leq 0 \quad(i \neq j)
$$

Utilizing the customary notation, it will be recalled that the focus of investigation in that section was the $\Gamma$-functions of Arthur:

$$
\Gamma_{F_{1}}^{F_{2}}\left(H, H_{0}\right)=\sum_{\left\{F: F_{1} \subset F \subset F_{2}\right\}}(-1)^{\#\left(F-F_{1}\right)} \chi_{F_{1}, F}(H) \cdot \chi^{F, F_{2}}\left(H-H_{0}\right) .
$$

Here is a complement to those considerations:

Proposition 3.1. We have

$$
\Gamma_{F_{1}}^{F_{2}}\left(H, H_{1}+H_{2}\right)=\sum_{\left\{F: F_{1} \subset F \subset F_{2}\right\}} \Gamma_{F_{1}}^{F}\left(H, H_{1}\right) \cdot \Gamma_{F}^{F_{2}}\left(H-H_{1}, H_{2}\right) .
$$

Proof. By definition, the right hand side of the purported equality is a sum over all $F, F^{\prime}, F^{\prime \prime}$ subject to

$$
F_{1} \subset F^{\prime} \subset F \subset F^{\prime \prime} \subset F_{2}
$$

of

$$
(-1)^{\#\left(F^{\prime}-F_{1}\right)} \cdot(-1)^{\#\left(F^{\prime \prime}-F\right)}
$$

times

$$
\chi_{F_{1}, F^{\prime}}(H) \cdot \chi^{F^{\prime}, F}\left(H-H_{1}\right) \cdot \chi_{F, F^{\prime \prime}}\left(H-H_{1}\right) \cdot \chi^{F^{\prime \prime}, F_{2}}\left(H-H_{1}-H_{2}\right) .
$$

Write the sign

$$
(-1)^{\#\left(F^{\prime}-F_{1}\right)} \cdot(-1)^{\#\left(F^{\prime \prime}-F\right)}
$$

in the form

$$
\left((-1)^{\#\left(F^{\prime}-F_{1}\right)} \cdot(-1)^{\#\left(F^{\prime \prime}-F\right)} \cdot(-1)^{\#\left(F^{\prime \prime}-F_{1}\right)}\right) \cdot(-1)^{\#\left(F^{\prime \prime}-F_{1}\right)}
$$

to get, alternatively

$$
(-1)^{\#\left(F-F^{\prime}\right)} \cdot(-1)^{\#\left(F^{\prime \prime}-F_{1}\right)} \text {. }
$$


Then our sum can be reexpressed as

$$
\begin{aligned}
& \sum_{\left\{F^{\prime}, F^{\prime \prime}: F_{1} \subset F^{\prime} \subset F^{\prime \prime} \subset F_{2}\right\}}(-1)^{\#\left(F^{\prime \prime}-F_{1}\right)} \chi_{F_{1}, F^{\prime}}(H) \cdot \chi^{F^{\prime \prime}, F_{2}}\left(H-H_{1}-H_{2}\right) \\
& \times \sum_{\left\{F: F^{\prime} \subset F \subset F^{\prime \prime}\right\}}(-1)^{\#\left(F-F^{\prime}\right)} \chi^{F^{\prime}, F}\left(H-H_{1}\right) \cdot \chi_{F, F^{\prime \prime}}\left(H-H_{1}\right) .
\end{aligned}
$$

But the sum over $F$ is just

$$
\sum_{\left\{F: F^{\prime} \subset F \subset F^{\prime \prime}\right\}}(-1)^{\#\left(F-F^{\prime}\right)} \tau_{F^{\prime}, F}\left(F^{\prime \prime}-F^{\prime}: H-H_{1}\right) \cdot \chi_{F, F^{\prime \prime}}\left(H-H_{1}\right)
$$

or still, by the Combinatorial Lemma of Langlands (cf. Proposition 2.5 of $[2-(b)])$,

$$
= \begin{cases}1 & \text { if } F^{\prime}=F^{\prime \prime} \\ 0 & \text { if } F^{\prime} \neq F^{\prime \prime} .\end{cases}
$$

Consequently, in the sum over $F^{\prime}, F^{\prime \prime}$, only the terms corresponding to $F^{\prime}=F^{\prime \prime}$ make a contribution. What is left, therefore, is precisely

$$
\Gamma_{F_{1}}^{F_{2}}\left(H, H_{1}+H_{2}\right)
$$

as desired.

REMARK. Using this result, it is easy to derive a dual to Proposition 2.3 of [2(d)], viz:

$$
\chi_{F_{1}, F_{2}}(H)=\sum_{\left\{F: F_{1} \subset F \subset F_{2}\right\}}(-1)^{\#\left(F-F_{1}\right)} \Gamma_{F_{1}}^{F}\left(H, H_{0}\right) \cdot \chi_{F, F_{2}}\left(H-H_{0}\right) .
$$

In fact, in the relation provided by the preceding proposition, begin by writing $H_{0}$ for $H_{1}$, next multiply both sides by $(-1)^{\#\left(F_{2}-F_{1}\right)}$, and finally let $H_{2} \rightarrow+\infty$ : It remains only to quote Proposition 2.5 of [2-(d)].

Let now

$$
p_{F_{1}}^{F_{2}}\left(H_{0}\right)=\int_{V_{F_{1}} \cap V\left(F_{2}\right)} \Gamma_{F_{1}}^{F_{2}}\left(H, H_{0}\right) d H .
$$

Then Proposition 2.11 in [2-(d)] tells us that $p_{F_{1}}^{F_{2}}\left(H_{0}\right)$ is a homogeneous polynomial in $H_{0}$ of degree

$$
\#\left(F_{2}-F_{1}\right)=\operatorname{dim}\left(V_{F_{1}} \cap V\left(F_{2}\right)\right) .
$$

Keeping to the terminology introduced in $\S 7$ of [2(f)], we shall call $p_{F_{1}}^{F_{2}}$ an Arthur polynomial. 
Proposition 3.2. We have

$$
p_{F_{1}}^{F_{2}}\left(H_{1}+H_{2}\right)=\sum_{\left\{F: F_{1} \subset F \subset F_{2}\right\}} p_{F_{1}}^{F}\left(H_{1}\right) \cdot p_{F}^{F_{2}}\left(H_{2}\right) .
$$

Proof. Indeed:

$$
\begin{aligned}
p_{F_{1}}^{F_{2}}\left(H_{1}+H_{2}\right)=\int_{V_{F_{1}} \cap V\left(F_{2}\right)} \Gamma_{F_{1}}^{F_{2}}\left(H, H_{1}+H_{2}\right) d H \\
=\sum_{\left\{F: F_{1} \subset F \subset F_{2}\right\}} \int_{V_{F_{1}} \cap V\left(F_{2}\right)} \Gamma_{F_{1}}^{F}\left(H, H_{1}\right) \cdot \Gamma_{F}^{F_{2}}\left(H-H_{1}, H_{2}\right) d H \\
=\sum_{\left\{F: F_{1} \subset F \subset F_{2}\right\}} \int_{V_{F_{1}} \cap V(F)} \Gamma_{F_{1}}^{F}\left(H, H_{1}\right) d H \\
\quad \times \int_{V_{F} \cap V\left(F_{2}\right)} \Gamma_{F}^{F_{2}}\left(H-H_{1}, H_{2}\right) d H \\
=\sum_{\left\{F: F_{1} \subset F \subset F_{2}\right\}} p_{F_{1}}^{F}\left(H_{1}\right) \cdot p_{F}^{F_{2}}\left(H_{2}\right),
\end{aligned}
$$

the contention.

It is to be observed that $p_{F_{1}}^{F_{2}}$ is recursively computable. To see this, let

$$
l_{2,1}=\#\left(F_{2}-F_{1}\right) \text {. }
$$

If $l_{2,1}=1$, so

$$
F_{2}=F_{1} \cup\{i\}, \quad \text { say, }
$$

then $\left\{\lambda_{i}^{F_{1}}\right\}$ is a basis for $V_{F_{1}} \cap V\left(F_{2}\right)$ (cf. Lemma 2.6 in [2-(b)]), thus, thanks to Propositions 2.5 and 2.7 in [2-(d)],

$$
p_{F_{1}}^{F_{2}}\left(H_{0}\right)=-\left(H_{0}(1,2), \lambda_{i}^{F_{1}} /\left\|\lambda_{i}^{F_{1}}\right\|\right) .
$$

If $l_{2,1}>1$, then, by the above,

$$
\begin{aligned}
p_{F_{1}}^{F_{2}}\left(H_{0}\right) & =2^{-l_{2,1}} \cdot p_{F_{1}}^{F_{2}}\left(2 H_{0}\right) \\
& =2^{-l_{2,1}} \cdot \sum_{\left\{F: F_{1} \subset F \subset F_{2}\right\}} p_{F_{1}}^{F}\left(H_{0}\right) \cdot P_{F}^{F_{2}}\left(H_{0}\right) \\
& =2^{-l_{2,1}} \cdot\left(2 p_{F_{1}}^{F_{2}}\left(H_{0}\right)+\sum_{\left\{F: F_{1} \subset F \subset F_{2}\right\}} p_{F_{1}}^{F}\left(H_{0}\right) \cdot p_{F}^{F_{2}}\left(H_{0}\right)\right),
\end{aligned}
$$

i.e.,

$$
p_{F_{1}}^{F_{2}}\left(H_{0}\right)=\frac{1}{2^{l_{2,1}}-2} \cdot \sum_{\left\{F: F_{1} \subseteq F \subset F G_{2}\right\}} p_{F_{1}}^{F}\left(H_{0}\right) \cdot p_{F}^{F_{2}}\left(H_{0}\right) .
$$


Maintaining the conventions of $\S 2$ of [2-(d)], Fourier transformation

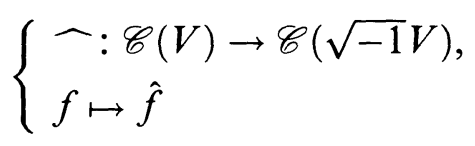

is the topological isomorphism defined by the rule

$$
\hat{f}(\Lambda)=\int_{V} f(H) e^{(\Lambda, H)} d H \quad(\Lambda \in \sqrt{-1} V) .
$$

There is a canonical injection $p \mapsto \partial_{p}$ of $\operatorname{Poly}(V)$ into the algebra of differential operators on $\sqrt{-1} V$ characterized by the condition

$$
\widehat{p f}=\partial_{p} \hat{f} \quad(f \in \mathscr{C}(V)) \text {. }
$$

LemMa 3.3. Let $p_{0}, p_{i}, q_{i}$ be elements of $\operatorname{Poly}(V)$-then the following are equivalent:

(i) $\forall H_{1}, H_{2} \in V: p_{0}\left(H_{1}+H_{2}\right)=\sum_{i} p_{i}\left(H_{1}\right) \cdot q_{i}\left(H_{2}\right)$;

(ii) $\forall \phi_{1}, \phi_{2} \in C^{\infty}(\sqrt{-1} V): \partial_{p_{0}}\left(\phi_{1} \phi_{2}\right)=\sum_{i}\left(\partial_{p_{l}} \phi_{1}\right) \cdot\left(\partial_{q_{l}} \phi_{2}\right)$.

Under (ii), the calculation

$$
\begin{aligned}
p_{0}\left(H_{1}+H_{2}\right) & =\left.\partial_{p_{0}}\left(e^{\left(\Lambda, H_{1}+H_{2}\right)}\right)\right|_{\Lambda=0} \\
& =\left.\partial_{p_{0}}\left(e^{\left(\Lambda, H_{1}\right)} e^{\left(\Lambda, H_{2}\right)}\right)\right|_{\Lambda=0} \\
& =\left.\left.\sum_{i}\left(\partial_{p_{l}} e^{\left(\Lambda, H_{1}\right)}\right)\right|_{\Lambda=0} \cdot\left(\partial_{q_{l}} e^{\left(\Lambda, H_{2}\right)}\right)\right|_{\Lambda=0} \\
& =\sum_{i} p_{i}\left(H_{1}\right) \cdot q_{i}\left(H_{2}\right)
\end{aligned}
$$

gives (i). The opposite direction can be established by using the "operational calculus" for Fourier transforms. However, it is perhaps preferable to cite a generality.

Sublemma. Suppose that $A$ is a commutative algebra over a field $k$. Let $p_{0}, p_{i}, q_{i}$ be elements of $k\left[t_{1}, \ldots, t_{n}\right]$ connected by the relation

$$
p_{0}(\mathbf{x}+\mathbf{y})=\sum_{i} p_{i}(\mathbf{x}) \cdot q_{i}(\mathbf{y}), \quad\left\{\begin{array}{l}
\mathbf{x}=\left(x_{1}, \ldots, x_{n}\right), \\
\mathbf{y}=\left(y_{1}, \ldots, y_{n}\right),
\end{array}\right.
$$

as polynomials in $2 n$-variables-then, for any collection $\boldsymbol{\partial}=\left(\partial_{1}, \ldots, \partial_{n}\right)$ of commuting $k$-linear derivations of $A$,

$$
p_{0}(\boldsymbol{\partial})(\alpha \beta)=\sum_{i}\left(p_{i}(\boldsymbol{\partial}) \alpha\right) \cdot\left(q_{i}(\boldsymbol{\partial}) \beta\right) \quad(\forall \alpha, \beta \in A) .
$$


Proof. Given $i$, put

$$
\begin{cases}\delta_{i}^{\prime}=\partial_{i} \otimes 1, & \boldsymbol{\delta}^{\prime}=\left(\delta_{1}^{\prime}, \ldots, \delta_{n}^{\prime}\right) \\ \delta_{i}^{\prime \prime}=1 \otimes \partial_{i}, & \boldsymbol{\delta}^{\prime \prime}=\left(\delta_{1}^{\prime \prime}, \ldots, \delta_{n}^{\prime \prime}\right),\end{cases}
$$

qua derivations of $A \otimes_{k} A$. Set $\delta_{i}=\delta_{i}^{\prime}+\delta_{i}^{\prime \prime}$-then the diagram

$$
\begin{array}{ll}
A \otimes_{k} A \stackrel{\pi}{\longrightarrow} A \\
\delta_{t} \quad \downarrow \quad(\pi(\alpha \otimes \beta)=\alpha \beta) \\
A \otimes_{k} A \stackrel{\pi}{\longrightarrow} A
\end{array}
$$

is commutative. Accordingly, with $\delta=\delta^{\prime}+\delta^{\prime \prime}$, the same is true of

$$
\begin{array}{ll}
A \otimes_{k} A \stackrel{\pi}{\longrightarrow} A \\
p_{0}(\boldsymbol{\delta}) \mid \\
A \otimes_{k} A \stackrel{\pi}{\longrightarrow} A
\end{array}
$$

Take now a tensor $\alpha \otimes \beta \in A \otimes_{k} A$-then, upon tracing its image in $A$ along the two possible routes supplied by this diagram, we obtain at once the claimed equality.

[Note: The converse to this purely algebraic statement is ordinarily false, a certain degree of "largeness" in $A$ being necessary.]

Needless to say, Lemma 3.3 is directly applicable to $p_{F_{1}}^{F_{2}}$, where, for the sake of simplicity, we write

$$
D_{F_{1}}^{F_{2}}=\partial_{p_{F_{1}}^{F_{2}}}
$$

$D_{*}^{*}$ being the special case when $F_{2}=\mathscr{L}, F_{1}=\varnothing$.

If $\phi$ is a $C^{\infty}$ function on $\sqrt{-1} V$, then its $A$-transform $A_{\phi}$ has been defined in $\S 2$ of [2-(d)] by the prescription

$$
A_{\phi}(\Lambda)=\sum_{\{F: F \subset \mathscr{L}\}} C_{F} \cdot(-1)^{\#(F)} \cdot\left[\frac{\phi\left(P_{F} \Lambda\right)}{\prod_{i \in F}\left(\Lambda, \lambda_{F}^{i}\right) \times \prod_{i \notin F}\left(\Lambda, \lambda_{i}^{F}\right)}\right]
$$

with the understanding that $\Lambda$ is ultraregular, i.e., lies on none of the hyperplanes determined by the $\lambda_{F}^{i}, \lambda_{i}^{F}$. It will be recalled that the thrust of Proposition 2.12 of that section was the fact that $A_{\phi}$ extends to a $C^{\infty}$ function on $\sqrt{-1} V$. If, in addition, $\phi=\hat{f}(f \in \mathscr{C}(V))$, then actually

$$
A_{\hat{f}}=(-1)^{\operatorname{dim}(V)} \hat{\Gamma}_{f}
$$


$\Gamma_{f}$ the $\Gamma$-transform of $f$, that is,

$$
\Gamma_{f}(H)=\int_{V} \Gamma_{*}^{*}\left(H, H_{0}\right) f\left(H_{0}\right) d H_{0}
$$

The next result helps to put all this into perspective.

Proposition 3.4. Let $\phi \in C^{\infty}(\sqrt{-1} V)$-then

$$
A_{\phi}(0)=(-1)^{\operatorname{dim}(V)} D_{*}^{*} \phi(0) \text {. }
$$

Proof. Because the question is local, we can assume that $\phi=\hat{f}$ $(f \in \mathscr{C}(V))$. But then

$$
\begin{aligned}
A_{\hat{f}}(0) & =(-1)^{\operatorname{dim}(V)} \hat{\Gamma}_{f}(0) \\
& =(-1)^{\operatorname{dim}(V)} \int_{V}\left(\int_{V} \Gamma_{*}^{*}\left(H, H_{0}\right) f\left(H_{0}\right) d H_{0}\right) d H \\
& =(-1)^{\operatorname{dim}(V)} \int_{V}\left(\int_{V} \Gamma_{*}^{*}\left(H, H_{0}\right) d H\right) f\left(H_{0}\right) d H_{0} \\
& =(-1)^{\operatorname{dim}(V)} \int_{V} p_{*}^{*}\left(H_{0}\right) f\left(H_{0}\right) d H_{0} \\
& =(-1)^{\operatorname{dim}(V)} D_{*}^{*} \hat{f}(0),
\end{aligned}
$$

as asserted.

In conclusion, it should be noted that there is an analogous result for $D_{F_{1}}^{F_{2}}$. Moreover, $\phi$ need only be $C^{\infty}$ in a neighborhood of the origin.

4. Extension of $\mathrm{II}_{\phi}$. The purpose of this section is to supplement the considerations of $\S 4$ in [2-(d)], the primary order of business being an explicit theorem of extension for the $\mathbf{I I}_{\phi}$-function (in the setting of Detroit families). As will be seen below, ultimately, the essential tool for doing this is Proposition 3.4 of the preceding section.

Let, therefore, $(V, \boldsymbol{\Phi})$ be a geometric g.r.s. of dimension $l$, say. Suppose that $\phi=\left\{\phi_{\mathscr{C}}\right\}$ is a $C^{\infty}$ Detroit family for which

$$
D_{\phi}=\bigcap \operatorname{dom}\left(\phi_{\mathscr{C}}\right)
$$

is a nonempty open subset of $\sqrt{-1} V$. By its very construction (cf. $\S 4$ in $[2-(d)])$,

$$
\mathbf{I I I}_{\phi}=\sum_{\mathscr{C}} \boldsymbol{\Theta}_{F_{0}(\mathscr{C})} \cdot \phi_{\mathscr{C}}
$$

is defined on the $\boldsymbol{\Phi}$-regular points in $D_{\phi}$, there even being the identity

$$
\text { III }_{\phi}=A_{\phi},
$$


valid formally on the $\boldsymbol{\Phi}$-ultraregular points in $D_{\phi}$ (cf. Theorem 4.4 of [2-(d)]). Here, of course,

$$
A_{\phi}=\sum_{\mathscr{C}} A_{\mathscr{C}, \phi_{\mathscr{C}}}
$$

The ostensible singularities that $\mathbf{I I}_{\phi}$ might possess are removable since it can be shown that $\mathrm{II}_{\phi}$ extends to a $C^{\infty}$ function on $D_{\phi}$ (cf. Proposition 4.6 in [2-(d)]).

[Note: Strictly speaking, this result was formulated and proved there only in the special case when $D_{\phi}$ was all of $\sqrt{-1} V$. However, to treat $D_{\phi} \neq \sqrt{-1} V$, it suffices to multiply the elements of $\phi$ by a common, $C^{\infty}$ cutoff function.]

The following question thus arises. Fix an arbitrary $\Lambda$ in $D_{\phi}$-then what, explicitly, is $\amalg_{\phi}(\Lambda)$ ? To answer this question, we shall first need to dispense with some geometric generalities.

Fix a point $\Lambda \in \sqrt{-1} V$. By $\mathscr{S}(\Lambda)$, we shall understand the set of all ordered triples $\left(V(F), \mathscr{C}_{F}, \mathscr{C}(F)\right)$, where

(1) $V(F)$ is the subspace of $V$ associated with a chamber set $F \subset \boldsymbol{\Phi}$ subject to

$$
\Lambda \perp V(F) ;
$$

(2) $\mathscr{C}_{F}$ is a chamber of $\boldsymbol{\Phi}_{F}$ having the property that

$$
\forall \lambda \in F_{0}\left(\mathscr{C}_{F}\right), \quad(\lambda, \Lambda) \neq 0 ;
$$

(3) $\mathscr{C}(F)$ is an arbitrary chamber of $\boldsymbol{\Phi}(F)$.

LEMMA 4.1. There is a canonical bijection between the chambers of $\boldsymbol{\Phi}$ and the elements of $\mathscr{S}(\Lambda)$.

Given a chamber of $\mathscr{C}$ of $\boldsymbol{\Phi}$, put

$$
F_{0}(\mathscr{C}: \Lambda)=\left\{\lambda \in F_{0}(\mathscr{C}):(\lambda, \Lambda)=0\right\} .
$$

Form, in the usual way, the spaces

$$
\left\{\begin{array}{l}
V\left(F_{0}(\mathscr{C}: \Lambda)\right), \\
V_{F_{0}(\mathscr{C}: \Lambda)},
\end{array}\right.
$$

denoting by

$$
\left\{\begin{array}{l}
\mathscr{C}(\Lambda) \\
\mathscr{C}_{\Lambda}
\end{array}\right.
$$

the chambers therein determined by $\mathscr{C}$. Obviously, $\Lambda$ is orthogonal to $V\left(F_{0}(\mathscr{C}: \Lambda)\right)$. Furthermore, because the short wall roots of $\mathscr{C}_{\Lambda}$ are the shortened elements of

$$
P \ldots\left(F_{0}(\mathscr{C})-F_{0}(\mathscr{C}: \Lambda)\right) \quad\left(\cdots=F_{0}(\mathscr{C}: \Lambda)\right),
$$


it is clear that

$$
\forall \lambda \in F_{0}\left(\mathscr{C}_{\Lambda}\right), \quad(\lambda, \Lambda) \neq 0
$$

So

$$
\mathscr{C} \rightarrow\left(V\left(F_{0}(\mathscr{C}: \Lambda)\right), \mathscr{C}_{\Lambda}, \mathscr{C}(\Lambda)\right) \in \mathscr{S}(\Lambda)
$$

On the other hand, consider any element $\left(V(F), \mathscr{C}_{F}, \mathscr{C}(F)\right)$ of $\mathscr{S}(\Lambda)$-then, on the basis of Proposition 3.15 of [2-(d)], there corresponds to the pair $\left(\mathscr{C}_{F}, \mathscr{C}(F)\right)$ a unique chamber $\mathscr{C}$ of $\boldsymbol{\Phi}$. In fact, in the notation of that result,

$$
\mathscr{C} \in \mathfrak{C}_{F}=\left\{\mathscr{C}: \mathscr{C}^{-} \cap V_{F}=\mathscr{C}_{F}^{-}\right\}
$$

the elements of

$$
P \ldots\left(F_{0}(\mathscr{C})-F_{0}(\mathscr{C}(F))\right) \quad\left(\cdots=F_{0}(\mathscr{C}(F))\right),
$$

when shortened, being $F_{0}\left(\mathscr{C}_{F}\right)$. If, as in the paragraph supra, we proceed from $\mathscr{E}$ to construct

$$
\left\{\begin{array} { l } 
{ V ( F _ { 0 } ( \mathscr { C } : \Lambda ) ) , } \\
{ V _ { F _ { 0 } ( \mathscr { C } : \Lambda ) } }
\end{array} \quad \left\{\begin{array}{l}
\mathscr{C}(\Lambda) \\
\mathscr{C}_{\Lambda}
\end{array}\right.\right.
$$

then

$$
\left\{\begin{array}{l}
V(F)=V\left(F_{0}(\mathscr{C}: \Lambda)\right), \quad\left\{\begin{array}{l}
\mathscr{C}(F)=\mathscr{C}(\Lambda), \\
V_{F}=V_{F_{0}(\mathscr{C}: \Lambda)},
\end{array} \mathscr{C}_{F}=\mathscr{C}_{\Lambda} .\right.
\end{array}\right.
$$

Consequently, the assignment

$$
\mathscr{C} \rightarrow\left(V\left(F_{0}(\mathscr{C}: \Lambda)\right), \mathscr{C}_{\Lambda}, \mathscr{C}(\Lambda)\right)
$$

is the bijection of our lemma.

For the sake of brevity, in what follows let us agree to write

$$
\begin{cases}V(\mathscr{C}: \Lambda) & \text { in place of } V\left(F_{0}(\mathscr{C}: \Lambda)\right) \\ P_{\mathscr{C}: \Lambda} & \text { in place of } P \ldots\left(\cdots=F_{0}(\mathscr{C}: \Lambda)\right)\end{cases}
$$

Returning to $\mathbf{I I I}_{\phi}$, rewrite

$$
\sum_{\mathscr{C}} \Theta_{F_{0}(\mathscr{C})} \cdot \phi_{\mathscr{C}}
$$

in the light of the above parametrization:

$$
\sum_{\left(V(\mathscr{C}: \Lambda), \mathscr{C}_{\Lambda}\right)} \sum_{\mathscr{C}(\Lambda)} \Theta_{F_{0}(\mathscr{C})} \cdot \phi_{\mathscr{C}} \quad\left(\Lambda \in D_{\phi}\right),
$$

where, as goes without saying, the notation is slightly deceptive. Our intention is to interpret the inner sum as a $\mathbf{I I}_{\text {? }}$, ? a Detroit family qua $V(\mathscr{C}: \Lambda)$. 
For this purpose, fix a

$$
\Lambda_{0} \in \sqrt{-1} P_{\mathscr{C}: \Lambda} V: \quad\left\|\Lambda_{0}-\Lambda\right\| \ll 1,
$$

so that $\Lambda_{0}$ and $\Lambda$ both have the same degree of regularity. Assuming that $H$ is in a suitable neighborhood of the origin in $\sqrt{-1} V(\mathscr{C}: \Lambda)$, attach to each chamber $\mathscr{C}(\Lambda)$ of $\Phi\left(F_{0}(\mathscr{C}: \Lambda)\right)$ the function

$$
\begin{aligned}
\phi_{\mathscr{C}(\Lambda)} & \left(\Lambda_{0}: H\right) \\
& =\frac{\left|D_{\mathscr{C}: \Lambda}\right|^{1 / 2}}{\prod_{\lambda_{1} \in F_{0}(\mathscr{C})-F_{0}(\mathscr{E}(\Lambda))}\left(\Lambda_{0}+H, \lambda_{i}\right)} \cdot \phi_{\mathscr{C}}\left(\Lambda_{0}+H\right),
\end{aligned}
$$

$D_{\mathscr{C}: \Lambda}$ being the determinant

$$
\operatorname{det}\left[\left(P_{\mathscr{C}: \Lambda} \lambda_{i}, P_{\mathscr{C}: \Lambda} \lambda_{j}\right): \lambda_{i}, \lambda_{j} \in F_{0}(\mathscr{C})-F_{0}(\mathscr{C}(\Lambda))\right] .
$$

The numerator of $\Theta_{F_{0}(\mathscr{E})}$ is equal to

$$
\left|\operatorname{det}\left[\left(\lambda_{i}, \lambda_{j}\right): \lambda_{i}, \lambda_{j} \in F_{0}(\mathscr{C}(\Lambda))\right]\right|^{1 / 2} \cdot\left|D_{\mathscr{C}: \Lambda}\right|^{1 / 2}
$$

Bearing in mind that

$$
\Lambda_{0} \perp V(\mathscr{C}: \Lambda)
$$

we therefore see that

$$
\begin{aligned}
\sum_{\mathscr{C}(\Lambda)} \Theta_{F_{0}(\mathscr{C})}\left(\Lambda_{0}+H\right) \cdot \phi_{\mathscr{C}}\left(\Lambda_{0}+H\right) \\
=\sum_{\mathscr{C}(\Lambda)} \Theta_{F_{0}(\mathscr{C}(\Lambda))}(H) \cdot \phi_{\mathscr{C}(\Lambda)}\left(\Lambda_{0}: H\right) .
\end{aligned}
$$

The latter relation suggests that we establish:

LeMma 4.2. The $\phi_{\mathscr{C}(\Lambda)}\left(\Lambda_{0}: ?\right)$ constitute a Detroit family $\phi_{\Lambda_{0}}$.

Proof. Suppose that $\mathscr{C}^{\prime}(\Lambda)$ and $\mathscr{C}^{\prime \prime}(\Lambda)$ are adjacent chambers in $V(\mathscr{C}: \Lambda)$ with $\mathscr{C}_{\lambda}^{\prime}=\mathscr{C}_{-\lambda}^{\prime \prime}$ as their common wall $\left(\lambda \in F_{0}\left(\mathscr{C}^{\prime}(\Lambda)\right),-\lambda \in\right.$ $F_{0}\left(\mathscr{C}^{\prime \prime}(\Lambda)\right)$ )-then the closure of $\mathscr{C}_{\lambda}^{\prime}=\mathscr{C}_{-\lambda}^{\prime \prime}$ is the intersection of the closures of $\mathscr{C}^{\prime}(\Lambda)$ and $\mathscr{C}^{\prime \prime}(\Lambda)$. Accordingly, since the correspondence

$$
\left\{\begin{array}{l}
\mathscr{C}^{\prime}(\Lambda) \rightarrow \mathscr{C}^{\prime} \\
\mathscr{C}^{\prime \prime}(\Lambda) \rightarrow \mathscr{C}^{\prime \prime}
\end{array} \text { per } \mathscr{C}_{\Lambda}\right.
$$

preserves adjacency,

$$
\phi_{\mathscr{C}^{\prime}}\left(\Lambda_{0}+H\right)=\phi_{\mathscr{C}^{\prime \prime}}\left(\Lambda_{0}+H\right) .
$$

Thus the issue reduces to the equality of

$$
\frac{\left|D_{\mathscr{C}^{\prime}: \Lambda}\right|^{1 / 2}}{\prod_{\lambda_{t}^{\prime} \in F_{0}\left(\mathscr{C}^{\prime}\right)-F_{0}\left(\mathscr{C}^{\prime}(\Lambda)\right)}\left(\Lambda_{0}+H, \lambda_{i}^{\prime}\right)}
$$


and

$$
\frac{\left|D_{\mathscr{C}^{\prime \prime}: \Lambda}\right|^{1 / 2}}{\prod_{\lambda_{1}^{\prime \prime} \in F_{0}\left(\mathscr{C}^{\prime \prime}\right)-F_{0}\left(\mathscr{C}^{\prime \prime}(\Lambda)\right)}\left(\Lambda_{0}+H, \lambda_{i}^{\prime \prime}\right)} .
$$

To resolve this point, consider roots

$$
\left\{\begin{array}{l}
\lambda_{i}^{\prime} \in F_{0}\left(\mathscr{C}^{\prime}\right)-\{\lambda\}, \\
\lambda_{i}^{\prime \prime} \in F_{0}\left(\mathscr{C}^{\prime \prime}\right)-\{-\lambda\},
\end{array}\right.
$$

which, when projected and shortened, give $\lambda_{0}$, say, i.e.,

$$
\begin{cases}\lambda_{0}=s_{i}^{\prime}\left(\lambda_{i}^{\prime}+t_{i}^{\prime} \lambda\right) & \left(s_{i}^{\prime}>0\right) \\ \lambda_{0}=s_{i}^{\prime \prime}\left(\lambda_{i}^{\prime \prime}+t_{i}^{\prime \prime} \lambda\right) & \left(s_{i}^{\prime \prime}>0\right) .\end{cases}
$$

Then

$$
\prod s_{i}^{\prime} \cdot\left|D_{\mathscr{C}: \Lambda}\right|^{1 / 2}
$$

is the same as

$$
\prod s_{i}^{\prime \prime} \cdot\left|D_{\mathscr{C}}{ }^{\prime \prime}: \Lambda\right|^{1 / 2}
$$

both being equal to

$$
\left|\operatorname{det}\left[\left(\lambda_{i}, \lambda_{j}\right): \lambda_{i}, \lambda_{j} \in F_{0}\left(\mathscr{C}_{\Lambda}\right)\right]\right|^{1 / 2} .
$$

Defining $t_{i}$ by the relation

$$
\lambda_{i}^{\prime \prime}=\left(s_{i}^{\prime} / s_{i}^{\prime \prime}\right) \lambda_{i}^{\prime}+t_{i} \lambda
$$

we have

$$
\left(\Lambda_{0}+H, \lambda_{i}^{\prime \prime}\right)=\left(s_{i}^{\prime} / s_{i}^{\prime \prime}\right)\left(\Lambda_{0}+H, \lambda_{i}^{\prime}\right)\left(\left(\Lambda_{0}+H, \lambda\right)=0\right) .
$$

Finally, putting everything together,

$$
\begin{aligned}
\frac{\text { Num }^{\prime}}{\text { Denom }^{\prime}} & =\frac{\prod s_{i}^{\prime} \cdot \mathrm{Num}^{\prime}}{\prod s_{i}^{\prime} \cdot \text { Denom }^{\prime}}=\frac{\prod s_{i}^{\prime \prime} \cdot \mathrm{Num}^{\prime \prime}}{\prod s_{i}^{\prime} \cdot \text { Denom }^{\prime}} \\
& =\frac{\prod s_{i}^{\prime \prime} \cdot \mathrm{Num}^{\prime \prime}}{\prod s_{i}^{\prime \prime} \cdot \text { Denom }^{\prime \prime}}=\frac{\mathrm{Num}^{\prime \prime}}{\text { Denom }^{\prime \prime}} .
\end{aligned}
$$

Hence the lemma.

Therefore

$$
\sum_{\mathscr{C}(\Lambda)} \Theta_{F_{0}(\mathscr{E}(\Lambda))}(H) \cdot \phi_{\mathscr{C}(\Lambda)}\left(\Lambda_{0}: H\right)
$$

can be viewed as

$$
\mathbf{I I}_{\phi_{\Lambda_{0}}}(H) \text {. }
$$

Because the second function admits a $C^{\infty}$ extension near the origin, the same must be true of the first. Now it is well known that if $M$ 
and $N$ are $C^{\infty}$ manifolds, then there is a canonical isomorphism of topological vector spaces

$$
C^{\infty}(M \times N) \simeq C^{\infty}\left(M ; C^{\infty}(N)\right) .
$$

Applying this fact to our situation allows us to assert that the map

$$
\left(\Lambda_{0}, H\right) \mapsto \sum_{\mathscr{C}(\Lambda)} \Theta_{F_{0}(\mathscr{C})}\left(\Lambda_{0}+H\right) \cdot \phi_{\mathscr{C}}\left(\Lambda_{0}+H\right)
$$

is $C^{\infty}$ near $(\Lambda, 0)$, the value of the extension being exactly $\mathbf{I I}_{\phi_{\Lambda}}(0)$ or still (cf. Theorem 4.4 of [2-(d)]) $A_{\phi_{\Lambda}}(0)$, computable by Proposition 3.4 .

The sought for extension theorem is then immediate:

THEOREM 4.3. Under the preceding hypotheses, $\mathbf{I I I}_{\phi}(\Lambda)$ is the sum over $\mathscr{C}$ of the

$$
\left.(-1)^{\#\left(F_{0}(\mathscr{C}(\Lambda))\right)} \cdot D_{*}^{F_{0}(\mathscr{C}(\Lambda))}\left[\frac{\left|D_{\mathscr{C}: \Lambda}\right|^{1 / 2}}{\prod_{\lambda_{1} \in F_{0}(\mathscr{C})-F_{0}(\mathscr{C}(\Lambda))}\left(?, \lambda_{i}\right)} \cdot \phi_{\mathscr{C}}\right]\right|_{\Lambda}
$$

In passing, observe that if $\Lambda$ is $\boldsymbol{\Phi}$-regular, then $\forall \mathscr{C}, F_{0}(\mathscr{C}(\Lambda))=\varnothing$ and $D_{*}^{\varnothing}=$ ID implying, as a result, that no differentiation is needed, which is to be expected.

A "vector-valued" variant of Theorem 4.3 will eventually be needed. To formulate it, let $\mathscr{E}$ be a finite dimensional vector space over $\mathbf{R}$ then

$$
\check{\mathscr{E}}=\operatorname{Hom}_{\mathbf{R}}(\mathscr{E}, \mathbf{C})
$$

is also a finite dimensional vector space over $\mathbf{R}$, there being a canonical pairing

$$
\langle ?, ?\rangle: \mathscr{E} \times \check{\mathscr{E}} \rightarrow \mathbf{C} .
$$

If $\forall \mathscr{C}$

$$
\phi_{\mathscr{C}}: \operatorname{dom}\left(\phi_{\mathscr{C}}\right) \rightarrow \check{\mathscr{E}},
$$

then the collection $\phi=\left\{\phi_{\mathscr{C}}\right\}$ is said to be a $C^{\infty}$ Detroit family with values in $\check{\mathscr{E}}$ if

$$
\forall T \in \mathscr{E}, \quad \phi_{T}=\left\{\left\langle T, \phi_{\mathscr{C}}\right\rangle\right\}
$$

is a $C^{\infty}$ Detroit family in the usual sense, where, to be specific,

$$
D_{\phi}=\bigcap \operatorname{dom}\left(\phi_{\mathscr{C}}\right)
$$

is a nonempty open subset of $\sqrt{-1} V$. Obviously, the theorem supra is applicable to each of the $\phi_{T}$. 
More generally, suppose that

$$
\mathbf{T}=\left\{T\left(V\left(F_{0}(\mathscr{C}: \Lambda)\right), \mathscr{C}_{\Lambda}, \mathscr{C}(\Lambda)\right)\right\}
$$

is a collection of $\mathscr{E}$-valued $C^{\infty}$ functions defined on a neighborhood of $\Lambda$, each of which is equal to $T$ at $\Lambda$ and having the property that $\forall \mathscr{C}^{\prime}, \mathscr{C}^{\prime \prime}$

$$
\begin{aligned}
& \left\{\begin{aligned}
V\left(F_{0}\left(\mathscr{C}^{\prime}: \Lambda\right)\right) & =V\left(F_{0}\left(\mathscr{C}^{\prime \prime}: \Lambda\right)\right), \\
\mathscr{C}_{\Lambda}^{\prime} & =\mathscr{C}_{\Lambda}^{\prime \prime}
\end{aligned}\right. \\
& \Rightarrow \\
& T\left(V\left(F_{0}\left(\mathscr{C}^{\prime}: \Lambda\right)\right), \mathscr{C}_{\Lambda}^{\prime}, \mathscr{C}^{\prime}(\Lambda)\right)=T\left(V\left(F_{0}\left(\mathscr{C}^{\prime \prime}: \Lambda\right)\right), \mathscr{C}_{\Lambda}^{\prime \prime}, \mathscr{C}^{\prime \prime}(\Lambda)\right) .
\end{aligned}
$$

Assigning to the symbol

$$
T\left(V(\mathscr{C}: \Lambda), \mathscr{C}_{\Lambda}\right)
$$

the evident interpretation, under natural assumptions on the domains, Lemma 4.2 implies that the

$$
\left\langle T\left(V(\mathscr{C}: \Lambda), \mathscr{C}_{\Lambda}\right), \phi_{\mathscr{C}(\Lambda)}\left(\Lambda_{0}: ?\right)\right\rangle
$$

constitute a Detroit family. Hence, in a suggestive notation:

THEOREM 4.3 (bis). Under the preceding hypotheses, $\mathbf{I I}_{\phi_{T}}(\Lambda)$ is the sum over $\mathscr{C}$ of the

$$
\left.(-1)^{\#\left(F_{0}(\mathscr{C}(\Lambda))\right)} \cdot D_{*}^{F_{0}(\mathscr{C}(\Lambda))}\left[\frac{\left|D_{\mathscr{C}: \Lambda}\right|^{1 / 2}}{\prod_{\lambda_{l} \in F_{0}(\mathscr{C})-F_{0}(\mathscr{C}(\Lambda))}\left(?, \lambda_{i}\right)} \cdot\left\langle T_{\mathscr{C}}, \phi_{\mathscr{C}}\right\rangle\right]\right|_{\Lambda} .
$$

[In fact, there is no loss of generality in making the evaluation at $\Lambda=$ 0 with $T_{\mathscr{C}}$ independent of $\mathscr{C}$, say $T_{\mathscr{C}}(\Lambda)=\mathbf{T}(\Lambda)$, where $T=\mathbf{T}(0)$. Call $\langle\mathbf{T}, \phi\rangle$ the $C^{\infty}$ Detroit family whose value at $\Lambda, \mathscr{C}$ is $\left\langle\mathbf{T}(\Lambda), \phi_{\mathscr{C}}(\Lambda)\right\rangle$ then

$$
\mathbf{I I}_{\phi_{T(\Lambda)}}(\Lambda)=\mathbf{I I I}_{\langle\mathrm{T}, \phi\rangle}(\Lambda)
$$

for all $\Phi$-regular $\Lambda$ and this persists to $\Lambda=0$.]

We shall also have to have a theorem of extension for the $\mathbf{I I I}_{\phi^{\cdot} \psi^{-}}$ function associated with the product of two $C^{\infty}$ Detroit families $\phi$ and $\psi$. To prepare for this, some additional notation will be required.

A subspace $W$ of $V$ will be termed special if $W=V(F)$ for some chamber set $F$. Let, therefore, $W$ be a special subspace of $V$-then attached to any Detroit family $\phi$ (not necessarily $C^{\infty}$ ) there is a Detroit family $\phi_{W}$ on $W^{\perp}$ (see Lemma 4.1 in [2-(d)]) and to each chamber $\mathscr{C}_{W}$ of $W^{\perp}$ a Detroit family $\phi\left(\mathscr{C}_{W}\right)$ on $W$ (see Lemma 4.2 in [2-(d)]). We 
remark that this notation is more appropriate than that initially used by us in [2-(d)]. It should also be pointed out that the correspondences in Propositions 3.14 and 3.15 of [2-(d)] are, in a sense, the "same". Without pursuing the matter, for all special $W$,

$$
\begin{aligned}
\left\{\mathscr{C}_{W}\right. & \left.: \text { chambers of } W^{\perp}\right\} \times\{\mathscr{C}(W) \text { : chambers of } W\} \\
& \simeq\left\{\mathscr{C} \text { : chambers of } V \text { st rel. int. }\left(\mathscr{C}^{-} \cap W^{\perp}\right) \neq \varnothing\right\} .
\end{aligned}
$$

Let now $\phi$ and $\psi$ be two $C^{\infty}$ Detroit families-then the product $\phi \cdot \psi$ is a $C^{\infty}$ Detroit family and:

THEOREM 4.4. Under the preceding hypotheses, $\mathbf{I I}_{\phi \cdot \psi}(0)$ is the sum over all special $W$ of the

$$
\sum_{\mathscr{C}_{W}}(-1)^{\#\left(F_{0}\left(\mathscr{C}_{W}\right)\right)} \cdot\left[\left(D_{*}^{F_{0}\left(\mathscr{C}_{W}\right)} \phi_{W, \mathscr{C}_{W}}\right)(0)\right] \cdot \mathbf{I I}_{\boldsymbol{\psi}\left(\mathscr{C}_{W}\right)}(0) .
$$

Proof. The unexplained notation being that of $\S 3$ in [2-(d)], write

$$
\begin{aligned}
\mathbf{I}_{\phi \cdot \boldsymbol{\psi}}(0) & =A_{\phi \cdot \boldsymbol{\psi}}(0) \\
& =\sum_{\mathscr{C}}(-1)^{\#\left(F_{0}(\mathscr{C})\right)} \cdot D_{*}^{F_{0}(\mathscr{C})}\left(\phi_{\mathscr{C}} \cdot \psi_{\mathscr{C}}\right)(0)
\end{aligned}
$$

or still (cf. §3)

$\sum_{\mathscr{C}} \sum_{\left.F \subset: F F_{0}(\mathscr{C})\right\}}(-1)^{\#\left(F_{0}(\mathscr{C})\right)-\#(F)} \cdot\left(D_{F}^{F_{0}(\mathscr{C})} \phi_{\mathscr{C}}\right)(0) \times(-1)^{\#(F)} \cdot\left(D_{*}^{F} \psi_{\mathscr{C}}\right)(0)$.

But

$$
\begin{aligned}
\sum_{\mathscr{C}} & \sum_{\left\{F: F \subset F_{0}(\mathscr{C})\right\}}=\sum_{F} \sum_{\mathscr{C} \in \mathfrak{C}_{0}(F)} \\
= & \sum_{W} \sum_{\mathscr{C}(W)} \sum_{\mathscr{C} \in \mathfrak{C}_{0}(F)}\left(F=F_{0}(\mathscr{C}(W))\right) \\
= & \sum_{W} \sum_{\mathscr{C}(W)} \sum_{\mathscr{C}_{W}}=\sum_{W} \sum_{\mathscr{C}_{W}} \sum_{\mathscr{C}(W)} .
\end{aligned}
$$

Moreover, if $\mathscr{C} \leftrightarrow\left(\mathscr{C}_{W}, \mathscr{C}(W)\right)$, then

$$
\forall \Lambda^{\perp} \in W^{\perp}, \quad D_{F_{0}(\mathscr{E}(W))}^{F_{0}(\mathscr{C})} \phi_{\mathscr{C}}\left(\Lambda^{\perp}\right)=D_{*}^{F_{0}\left(\mathscr{C}_{W}\right)} \phi_{W, \mathscr{C}_{W}}\left(\Lambda^{\perp}\right) .
$$

In particular, this is true at $\Lambda^{\perp}=0$. On the other hand,

$$
\psi_{\mathscr{C}}=\psi\left(\mathscr{C}_{W}\right)_{\mathscr{C}(W)}
$$


Accordingly, $\mathbf{I I}_{\phi \cdot \psi}(0)$ is the sum over all special $W$ of the

$$
\begin{aligned}
\sum_{\mathscr{C}_{W}}(-1)^{\#\left(F_{0}\left(\mathscr{C}_{W}\right)\right)} \cdot\left[\left(D_{*}^{F_{0}\left(\mathscr{C}_{W}\right)} \phi_{W, \mathscr{C}_{W}}\right)(0)\right] \\
\cdot \sum_{\mathscr{E}(W)}(-1)^{\#\left(F_{0}(\mathscr{C}(W))\right)} \cdot\left(D_{*}^{F_{0}(\mathscr{C}(W))} \psi\left(\mathscr{C}_{W}\right)_{\mathscr{C}(W)}\right)(0) .
\end{aligned}
$$

Since the sum over $\mathscr{C}(W)$ can be collapsed to $\mathbf{I I}_{\psi\left(\mathscr{C}_{W}\right)}(0)$, we are done.

REMARK. The preceding theorem retains its validity when, e.g., $\phi$ and $\psi$ are $C^{\infty}$ operator-valued Detroit families on a finite dimensional Hilbert space.

In general, if $f$ is a $C^{\infty}$ function, then it is clear that

$$
\text { III }_{f \cdot \phi}=f \cdot \text { II }_{\phi} \text {. }
$$

This simple observation, when combined with the above, leads to an important principle of invariance, viz.:

Proposition 4.5. Suppose that $\left\{f\left(W, \mathscr{C}_{W}\right)\right\}$ is a family of $C^{\infty}$ functions such that

$$
f\left(W, \mathscr{C}_{W}\right)(0)=1 .
$$

Let $\phi$ and $\psi$ be $C^{\infty}$ Detroit families-then $\mathbf{I I}_{\phi \cdot \psi}(0)$ is the sum over all special $W$ of the

$$
\sum_{\mathscr{C}_{W}}(-1)^{\#\left(F_{0}\left(\mathscr{C}_{W}\right)\right)} \cdot\left[\left(D_{*}^{F_{0}\left(\mathscr{C}_{W}\right)} \phi_{W, \mathscr{C}_{W}}\right)(0)\right] \cdot \mathbf{I I I}_{f\left(W, \mathscr{C}_{W}\right) \cdot \boldsymbol{\psi}\left(\mathscr{C}_{W}\right)}(0) .
$$

[Note: An operator-valued variant of this assertion is also true provided that we assume $f\left(W, \mathscr{C}_{W}\right)(0)$ is the identity map.]

Let us add a small aside that will come into play during the cancellation.

Proposition 4.6. Suppose that $\phi=\left\{\phi_{\mathscr{C}}\right\}$ is a Schwartz Detroit family in the sense that each $\phi_{\mathscr{C}} \in \mathscr{C}(\sqrt{-1} V)$-then

$$
\mathbf{I I}_{\phi} \in \mathscr{C}(\sqrt{-1} V) \text {. }
$$

In fact, if we let

$$
\phi_{\mathscr{C}}^{N}(\Lambda)=\left(1+\|\Lambda\|^{2}\right)^{N} \cdot \phi_{\mathscr{C}}(\Lambda),
$$

then $\phi_{\mathscr{C}}^{N} \in \mathscr{C}(\sqrt{-1} V)$ and, of course,

$$
\mathbf{I I I}_{\phi}=\left(1+\|\Lambda\|^{2}\right)^{-N} \cdot \mathbf{I I}_{\phi^{N}} .
$$

Therefore, taking into account the Leibnitz rule, it would be enough to prove that if each $\phi_{\mathscr{C}} \in \mathscr{C}(\sqrt{-1} V)$, then $D \mathrm{II}_{\phi}$ is bounded for every 
translation invariant differential operator $D$. But this follows from:

LeMMA 4.7. Fix D. Let $\phi \in \mathscr{C}(\sqrt{-1} V)$-then $D A_{\phi}$ is bounded on $\sqrt{-1} V$.

[One need only inspect the proof of Lemma 2.13 in [2(d)].]

5. Manipulation of the leading term $(P=G)$. The purpose of this section is to study the leading term of the inner product of two truncated Eisenstein series in the boldface picture. Lest we lose sight of our objective, let us bear in mind that the main application of all this will be to the inner products

$$
\left(Q^{\mathbf{H}} \mathbf{E}\left(P_{i} \mid A_{i}: \mathbf{e}_{m}^{i}: \Lambda_{i}: ?\right), Q^{\mathbf{H}} \mathbf{E}\left(P_{i} \mid A_{i}: \mathbf{e}_{n}^{i}: \Lambda_{i}: ?\right)\right)
$$

entering into the calculation of the integral

from $\S 2$.

$$
\int_{G / \Gamma} Q_{(1)}^{\mathbf{H}} Q_{(2)}^{\mathbf{H}} K_{\alpha}\left(x, x: \boldsymbol{\sigma}_{i}, \Lambda_{i}\right) d_{G}(x)
$$

Fix an association class $\mathscr{C}$ of $\Gamma$-cuspidal split parabolic subgroups of $G$. Decompose $\mathscr{E}$ into $G$-conjugacy classes $\mathscr{C}_{i}$ :

$$
\mathscr{C}=\coprod_{i} \mathscr{C}_{i} \quad\left(\mathscr{C}_{i}=G \cdot\left\{P_{i}\right\} \cap \mathscr{C}\right)
$$

Given

$$
\left\{\begin{array}{l}
\boldsymbol{\Phi}=\left(\boldsymbol{\Phi}_{1}, \ldots, \boldsymbol{\Phi}_{r_{t}}\right) \\
\boldsymbol{\Psi}=\left(\boldsymbol{\Psi}_{1}, \ldots, \boldsymbol{\Psi}_{r_{\iota}}\right)
\end{array} \in \mathscr{E}\left(\delta, \boldsymbol{\sigma}_{i}\right)=\sum_{\mu} \bigoplus \mathscr{E}\left(\delta, \mathscr{O}_{i \mu}\right)\right.
$$

write

$$
(\boldsymbol{\Phi}, \boldsymbol{\Psi})_{\mathscr{C}_{i}}=\left(\left(\boldsymbol{\Phi}_{1}, \boldsymbol{\Psi}_{1}\right), \ldots,\left(\boldsymbol{\Phi}_{r_{i}}, \boldsymbol{\Psi}_{r_{i}}\right)\right)
$$

a vector in $\mathbf{C}^{r_{i}}$. Per $\Lambda_{i} \in \check{\mathfrak{a}}_{i}+\sqrt{-1} \check{\mathfrak{a}}_{i}$, put

$$
\mathbf{e}\left(\mathscr{C}_{i}: \mathbf{H}, \Lambda_{i}\right)=\left(\exp \left(\left\langle\mathbf{H}\left(P_{i \mu}\right), I\left(P_{i \mu}, P_{i}\right) \Lambda_{i}\right\rangle\right)\right) \quad(\mathbf{H} \in \mathfrak{a}),
$$

another vector in $\mathbf{C}^{r_{1}}$. Accordingly,

$$
\left\langle\mathbf{e}\left(\mathscr{C}_{i}: \mathbf{H}, \Lambda_{i}\right),(\boldsymbol{\Phi}, \Psi) \mathscr{\mathscr { C }}_{i}\right\rangle \in \mathbf{C} .
$$

To illustrate the utility of this notation, let $\mathscr{C}_{i_{1}}$ and $\mathscr{C}_{i_{2}}$ be $G$-conjugacy classes in $\mathscr{C}$ with

$$
\left\{\begin{array}{l}
\left(P_{1}, S_{1} ; A_{1}\right) \in \mathscr{C}_{i_{1}} \\
\left(P_{2}, S_{2} ; A_{2}\right) \in \mathscr{C}_{i_{2}} .
\end{array}\right.
$$

Take elements

$$
\left\{\begin{array}{l}
\boldsymbol{\Phi}_{1} \in \mathscr{E}\left(\delta, \boldsymbol{\theta}_{i_{1}}\right) \\
\boldsymbol{\Phi}_{2} \in \mathscr{E}\left(\delta, \boldsymbol{\sigma}_{i_{2}}\right)
\end{array} \quad\left(\boldsymbol{\sigma}_{i_{1}} \leftrightarrow \boldsymbol{\sigma}_{i_{2}}\right),\right.
$$


and form the Eisenstein series

$$
\begin{cases}\mathbf{E}\left(P_{1} \mid A_{1}: \boldsymbol{\Phi}_{1}: \Lambda_{1}: ?\right) & \left(\Lambda_{1} \in \check{\mathfrak{a}}_{1}+\sqrt{-1} \check{\mathfrak{a}}_{1}\right), \\ \mathbf{E}\left(P_{2} \mid A_{2}: \mathbf{\Phi}_{2}: \Lambda_{2}: ?\right) & \left(\Lambda_{2} \in \check{\mathfrak{a}}_{2}+\sqrt{-1} \check{\mathfrak{a}}_{2}\right) .\end{cases}
$$

Then, suppossing that $\Lambda_{1}$ and $\Lambda_{2}$ are pure imaginary and in general position, the "leading term" of the inner product

$$
\left(Q^{\mathbf{H}} \mathbf{E}\left(P_{1} \mid A_{1}: \boldsymbol{\Phi}_{1}: \Lambda_{1}: ?\right), Q^{\mathbf{H}} \mathbf{E}\left(P_{2} \mid A_{2}: \boldsymbol{\Phi}_{2}: \Lambda_{2}: ?\right)\right) \quad\left(\mathbf{H} \in \mathfrak{a}_{Q}\right)
$$

is the sum

$$
(-1)^{l} \cdot \operatorname{vol}(\mathscr{C}) \cdot \sum_{i=1}^{r} \sum_{w_{i: 1} \in W\left(A_{l}, A_{1}\right)} \sum_{w_{i \cdot 2} \in W\left(A_{l}, A_{2}\right)}
$$

of the

$$
\begin{aligned}
\left\langle\mathbf { e } \left(\mathscr{C}_{i}: \mathbf{H}, w_{i: 1} \Lambda_{1}+\right.\right. & \left.w_{i: 2} \bar{\Lambda}_{2}\right), \\
\left(\mathbf { c } \left(P_{i} \mid A_{i}:\right.\right. & \left.\left.\left.P_{1} \mid A_{1}: w_{i: 1}: \Lambda_{1}\right) \boldsymbol{\Phi}_{1}, \mathbf{c}\left(P_{i}\left|A_{i}: P_{2}\right| A_{2}: w_{i: 2}: \Lambda_{2}\right) \boldsymbol{\Phi}_{2}\right) \mathscr{C}_{t}\right\rangle \\
& \times\left(1 / \prod_{\lambda_{t}}\left(w_{i: 1} \Lambda_{1}+w_{i: 2} \bar{\Lambda}_{2}, \lambda_{i}\right)\right)
\end{aligned}
$$

The details may be found in $\S 8$ of [2(d)]. Needless to say, the phrase "leading term" is suggested by the fact that what remains of the inner product is in some sense negligible (cf. $\S 10$ of [2(d)]).

It is true, but not quite immediate, that

$$
\sum_{i=1}^{r} \sum_{w_{i 1} \in W\left(A_{1}, A_{1}\right)} \sum_{w_{i: 2} \in W\left(A_{1}, A_{2}\right)} \cdots
$$

extends to a holomorphic function of $\left(\Lambda_{1}, \bar{\Lambda}_{2}\right)$ on $\sqrt{-1} \check{\mathfrak{a}}_{1} \times \sqrt{-1} \check{\mathfrak{a}}_{2}$. To establish this, we may proceed via an argument entirely analogous to the one employed in $\S 9$ of [2-(d)], the key again being the theory of Detroit families. Actually, a more general result is valid in that it is possible to produce $C^{\infty}$ extensions in a variety of ways. While a discussion in extenso is certainly not necessary, we had best at least indicate the essential ideas as they lead to formulae of no small importance for what is to follow.

Let $i$ be any index between 1 and $r$. Given

$$
\left\{\begin{array}{l}
w_{i: 1} \in W\left(A_{i}, A_{1}\right), \\
w_{i: 2} \in W\left(A_{i}, A_{2}\right),
\end{array}\right.
$$

write

$$
Ю_{i}\left(\Lambda_{1}, \Lambda_{2}: w_{i: 1}, w_{i: 2} ; \mathbf{\Phi}_{1}, \mathbf{\Phi}_{2}: \mathbf{H}\right)
$$


in place of

$$
\begin{aligned}
& \left\langle\mathbf{e}\left(\mathscr{C}_{i}: \mathbf{H}, w_{i: 1} \Lambda_{1}+w_{i: 2} \bar{\Lambda}_{2}\right),\right. \\
& \left.\quad\left(\mathbf{c}\left(P_{i}\left|A_{i}: P_{1}\right| A_{1}: w_{i: 1}: \Lambda_{1}\right) \boldsymbol{\Phi}_{1}, \mathbf{c}\left(P_{i}\left|A_{i}: P_{2}\right| A_{2}: w_{i: 2}: \Lambda_{2}\right) \boldsymbol{\Phi}_{2}\right) \mathscr{\mathscr { C }}_{i}\right\rangle .
\end{aligned}
$$

Then the $\mathrm{F}_{i}$ are meromorphic functions of $\left(\Lambda_{1}, \bar{\Lambda}_{2}\right)$ which are, moreover, holomorphic on $\sqrt{-1} \check{a}_{1} \times \sqrt{-1} \check{a}_{2}$, this also being the case of

$$
\sum_{i=1}^{r} \sum_{w_{i: 1} \in W\left(A_{l}, A_{1}\right)} \sum_{w_{i: 2} \in W\left(A_{i}, A_{2}\right)} \frac{\wp_{i}\left(\Lambda_{1}, \Lambda_{2} ; w_{i: 1}, w_{i: 2} ; \boldsymbol{\Phi}_{1}, \boldsymbol{\Phi}_{2}: \mathbf{H}\right)}{\prod_{\lambda_{l}}\left(w_{i: 1} \Lambda_{1}+w_{i: 2} \bar{\Lambda}_{2}, \lambda_{i}\right)} .
$$

Setting $A=A_{1}$ and thus agreeing to write $w_{i}$ instead of $w_{i: 1}$, for any $w_{12} \in W\left(A_{1}, A_{2}\right)$, put

$$
\begin{aligned}
& \Upsilon\left(\Lambda_{1}, \Lambda_{2} ; w_{12} ; \boldsymbol{\Phi}_{1}, \boldsymbol{\Phi}_{2}: \mathbf{H}\right) \\
& \quad=\sum_{i=1}^{r} \sum_{w_{i} \in W\left(A_{i}, A\right)} \frac{Ю_{i}\left(\Lambda_{1}, \Lambda_{2} ; w_{i}, w_{i} w_{12} ; \boldsymbol{\Phi}_{1}, \mathbf{\Phi}_{2}: \mathbf{H}\right)}{\prod_{\lambda_{i}}\left(w_{i} \Lambda_{1}+w_{i} w_{12} \bar{\Lambda}_{2}, \lambda_{i}\right)} .
\end{aligned}
$$

In terms of this parametrization, our problem will be the $C^{\infty}$ prolongment of

$$
\sum_{w_{12} \in W\left(A_{1}, A_{2}\right)} \Upsilon\left(\Lambda_{1}, \Lambda_{2} ; w_{12} ; \mathbf{\Phi}_{1}, \mathbf{\Phi}_{2}: \mathbf{H}\right)
$$

to $\sqrt{-1} \check{\mathfrak{a}}$ under the assumption that $\Lambda_{1}$ and $\Lambda_{2}$ are suitable functions of $\Lambda \in \sqrt{-1}$ ă (cf. infra).

Since it suffices to analyze each constituent of the sum separately, fix a $w_{12}$. The space a comes supplied with the structure of a g.r.s., the chambers being in a natural one-to-one correspondence with the elements of the set

$$
\coprod_{i=1}^{r}\{i\} \times W\left(A_{i}, A\right)
$$

as follows from Lemma 2 on p. 67 of TES. Put

$$
\mathscr{E}=\mathscr{E}\left(\delta, \boldsymbol{\theta}_{i_{1}}\right) \otimes_{\mathbf{R}} \mathscr{E}\left(\delta, \boldsymbol{\theta}_{i_{2}}\right)
$$

Let

$$
\left\{\begin{array}{l}
\Lambda_{1}: \sqrt{-1} \check{\mathfrak{a}} \rightarrow \sqrt{-1} \check{\mathfrak{a}}_{1}, \\
\Lambda_{2}: \sqrt{-1} \check{\mathfrak{a}} \rightarrow \sqrt{-1} \check{\mathfrak{a}}_{2}
\end{array}\right.
$$

be $C^{\infty}$ functions on $\sqrt{-1}$ ă satisfying the condition

$$
\Lambda=\Lambda_{1}(\Lambda)+w_{12} \bar{\Lambda}_{2}(\Lambda) \quad(\Lambda \in \sqrt{-1} \check{\mathfrak{a}}) .
$$

For each pair $\left(i, w_{i}\right)$, define

$$
\phi_{\left(i, w_{\imath}\right)}: \sqrt{-1} \check{\mathfrak{a}} \rightarrow \check{\mathscr{E}}
$$


by the rule

$$
\left\langle\boldsymbol{\Phi}_{1} \otimes \boldsymbol{\Phi}_{2}, \phi_{\left(i, w_{i}\right)}(\Lambda)\right\rangle=\mathrm{Ю}_{i}\left(\Lambda_{1}(\Lambda), \Lambda_{2}(\Lambda) ; w_{i}, w_{i} w_{12} ; \boldsymbol{\Phi}_{1}, \boldsymbol{\Phi}_{2}: \mathbf{H}\right) .
$$

The $\phi_{\left(i, w_{i}\right)}$ are obviously $C^{\infty}$. Moreover:

LemMa 5.1. The $\phi_{\left(i, w_{\imath}\right)}$ constitute a Detroit family $\phi\left(w_{12}\right)$.

[The verification is essentially standard, being based on the functional equations for the c-functions (cf., e.g., pp. 475-480 in [2-(b)]). Therefore, we can skip over some of the details. If $\left(i^{\prime}, w_{i^{\prime}}\right)$ and $\left(i^{\prime \prime}, w_{i^{\prime \prime}}\right)$ correspond to adjacent chambers with common wall in $\check{\mathfrak{a}}_{\lambda}$, then the question is the equality of the restrictions of

$$
\phi_{\left(i^{\prime}, w^{\prime}\right)} \text { and } \phi_{\left(i^{\prime \prime}, w_{t^{\prime \prime}}\right)}
$$

to $\sqrt{-1} \check{\mathfrak{a}}_{\lambda}$. This said, let us change the notation and deal, as is permissible, with pairs $\left(i, w_{i}\right)$ and $\left(j, w_{\lambda} w_{i}\right)$, where $w_{\lambda} \in W\left(A_{j}, A_{i}\right)$ is a simple reflection. We may then attach to $\lambda$, in the usual way, a $\Gamma$-cuspidal parabolic $P_{\lambda} \succeq P_{i}, P_{j}$ with $P_{i}^{\dagger}, P_{j}^{\dagger}$ maximal. Of course, tacitly, certain adjustments may be necessary to achieve this. Call $\mathscr{C}_{\lambda}$ the $G$-conjugacy class of $\Gamma$-cuspidals determined by $P_{\lambda}$. Define maps

$$
\left\{\begin{array}{l}
\operatorname{red}\left(\mathscr{C}_{\lambda}, \mathscr{C}_{i}\right): \mathbf{C}^{r_{\iota}} \rightarrow \mathbf{C}^{r_{\lambda}}, \\
\operatorname{red}\left(\mathscr{C}_{\lambda}, \mathscr{C}_{j}\right): \mathbf{C}^{r_{\jmath}} \rightarrow \mathbf{C}^{r_{\lambda}}
\end{array}\right.
$$

by stipulating that the $l_{\lambda}$-component of

$$
\left\{\begin{array} { l } 
{ ( z _ { 1 } , \ldots , z _ { r _ { l } } ) } \\
{ ( z _ { 1 } , \ldots , z _ { r _ { j } } ) }
\end{array} \text { is to be } \left\{\begin{array}{l}
\sum_{\mu} z_{\mu} \\
\sum_{\nu} z_{\nu},
\end{array}\right.\right.
$$

the sum running over the $\left\{\begin{array}{l}\mu \\ \nu\end{array}\right.$ for which

$$
\left\{\begin{array}{l}
P_{i \mu}, \\
P_{j \nu}
\end{array}\right.
$$

is a dominated predecessor of $P_{i_{\lambda}}\left(1 \leq l_{\lambda} \leq r_{\lambda}\right)$. Assume now that $\Lambda$ lies in $\sqrt{-1} \check{\mathfrak{a}}_{\lambda}$-then

$$
\left\langle\boldsymbol{\Phi}_{1} \otimes \boldsymbol{\Phi}_{2}, \phi_{\left(i, w_{\imath}\right)}(\Lambda)\right\rangle
$$

is the result of pairing

$$
\mathbf{e}\left(\mathscr{C}_{\lambda}: \mathbf{H}, w_{i} \Lambda\right)
$$

with

$$
\begin{aligned}
\operatorname{red}\left(\mathscr{C}_{\lambda}, \mathscr{C}_{i}\right)\left(\mathbf{c}\left(P_{i}\left|A_{i}: P_{1}\right| A_{1}: w_{i}: \Lambda_{1}(\Lambda)\right) \boldsymbol{\Phi}_{1}\right. \\
\left.\quad \mathbf{c}\left(P_{i}\left|A_{i}: P_{2}\right| A_{2}: w_{i} w_{12}: \Lambda_{2}(\Lambda)\right) \boldsymbol{\Phi}_{2}\right)_{\mathscr{C}_{i}}
\end{aligned}
$$


while

$$
\left\langle\boldsymbol{\Phi}_{1} \otimes \boldsymbol{\Phi}_{2}, \phi_{\left(j, w_{\imath} w_{\imath}\right)}(\Lambda)\right\rangle
$$

is the result of pairing

$$
\mathbf{e}\left(\mathscr{C}_{\lambda}: \mathbf{H}, w_{\lambda} w_{i} \Lambda\right)
$$

with

$$
\begin{aligned}
\operatorname{red}\left(\mathscr{C}_{\lambda}, \mathscr{C}_{j}\right)\left(\mathbf{c}\left(P_{j}\left|A_{j}: P_{1}\right| A_{1}: w_{\lambda} w_{i}: \Lambda_{1}(\Lambda)\right) \boldsymbol{\Phi}_{1},\right. \\
\left.\quad \mathbf{c}\left(P_{j}\left|A_{j}: P_{2}\right| A_{2}: w_{\lambda} w_{i} w_{12}: \Lambda_{2}(\Lambda)\right) \boldsymbol{\Phi}_{2}\right) \mathscr{C}_{j} .
\end{aligned}
$$

Because $w_{i} \Lambda=w_{\lambda} w_{i} \Lambda$, we have only to check the equality of

$$
\operatorname{red}\left(\mathscr{C}_{\lambda}, \mathscr{C}_{i}\right)(\ldots)_{\mathscr{C}_{i}} \text { and } \operatorname{red}\left(\mathscr{C}_{\lambda}, \mathscr{C}_{j}\right)(\ldots)_{\mathscr{C}_{j}}
$$

Owing to the functional equations,

$$
\begin{aligned}
\mathbf{c}\left(P_{j}\left|S_{j}: P_{1}\right| A_{1}: w_{\lambda} w_{i}: \Lambda_{1}(\Lambda)\right) \\
=\mathbf{c}\left(P_{j}\left|A_{j}: P_{i}\right| A_{i}: w_{\lambda}: w_{i} \Lambda_{1}(\Lambda)\right) \\
\quad \cdot \mathbf{c}\left(P_{i}\left|A_{i}: P_{1}\right| A_{1}: w_{i}: \Lambda_{1}(\Lambda)\right)
\end{aligned}
$$

and

$$
\begin{aligned}
\mathbf{c}\left(P_{j} \mid A_{j}:\right. & \left.P_{2} \mid A_{2}: w_{\lambda} w_{i} w_{12}: \Lambda_{2}(\Lambda)\right) \\
= & \mathbf{c}\left(P_{j}\left|A_{j}: P_{i}\right| A_{i}: w_{\lambda}: w_{i} w_{12} \Lambda_{2}(\Lambda)\right) \\
& \cdot \mathbf{c}\left(P_{i}\left|A_{i}: P_{2}\right| A_{2}: w_{i} w_{12}: \Lambda_{2}(\Lambda)\right)
\end{aligned}
$$

Let

$$
\left\{\begin{array}{l}
\boldsymbol{\Phi}_{1}^{i}=\mathbf{c}\left(P_{i}\left|A_{i}: P_{1}\right| A_{1}: w_{i}: \Lambda_{1}(\Lambda)\right) \boldsymbol{\Phi}_{1} \\
\boldsymbol{\Phi}_{2}^{i}=\mathbf{c}\left(P_{i}\left|A_{i}: P_{2}\right| A_{2}: w_{i} w_{12}: \Lambda_{2}(\Lambda)\right) \boldsymbol{\Phi}_{2}
\end{array} \in \mathscr{E}\left(\delta, \boldsymbol{\theta}_{i}\right) .\right.
$$

Then the claim is that

$$
\operatorname{red}\left(\mathscr{C}_{\lambda}, \mathscr{C}_{i}\right)\left(\boldsymbol{\Phi}_{1}^{i}, \boldsymbol{\Phi}_{2}^{i}\right)_{\mathscr{C}_{i}}
$$

is the same as

$$
\begin{aligned}
\operatorname{red}\left(\mathscr{C}_{\lambda}, \mathscr{C}_{j}\right)\left(\mathbf{c}\left(P_{j}\left|A_{j}: P_{i}\right| A_{i}: w_{\lambda}: w_{i} \Lambda_{1}(\Lambda)\right) \boldsymbol{\Phi}_{1}^{i},\right. \\
\\
\left.\quad \mathbf{c}\left(P_{j}\left|A_{j}: P_{i}\right| A_{i}: w_{\lambda}: w_{i} w_{12} \Lambda_{2}(\Lambda)\right) \boldsymbol{\Phi}_{2}^{i}\right)_{\mathscr{C}_{j}} .
\end{aligned}
$$

Taking into account the fact that

$$
\Lambda=\Lambda_{1}(\Lambda)+w_{12} \bar{\Lambda}_{2}(\Lambda),
$$

we reduce without difficulty to a purely rank one situation, which can then be handled directly.] 
Our notation is incomplete in that neither the functions $\Lambda_{1}(\Lambda)$ and $\Lambda_{2}(\Lambda)$ nor $\mathbf{H}$ itself are reflected by the symbol $\phi\left(w_{12}\right)$. Keeping this in mind, given $\boldsymbol{\Phi}_{1}$ and $\boldsymbol{\Phi}_{2}$, from the

$$
\left\langle\boldsymbol{\Phi}_{1} \otimes \boldsymbol{\Phi}_{2}, \phi_{\left(i, w_{\imath}\right)}\right\rangle
$$

we may form

$$
\text { III }_{\phi\left(w_{12}\right)}\left(\Lambda ; \boldsymbol{\Phi}_{1}, \boldsymbol{\Phi}_{2}: \mathbf{H}\right) \text {, }
$$

a $C^{\infty}$ function of $\Lambda$ in $\sqrt{-1} \check{\mathfrak{a}}$ (cf. Proposition 4.6 in [2-(d)]). Modulo the constant $\operatorname{vol}(\mathscr{C})$, it is this function which provides a $C^{\infty}$ extension of

$$
\Upsilon\left(\Lambda_{1}(\Lambda), \Lambda_{2}(\Lambda) ; w_{12} ; \boldsymbol{\Phi}_{1}, \boldsymbol{\Phi}_{2}: \mathbf{H}\right)
$$

to all of $\sqrt{-1}$ ă.

Here is an illustration. Let us assume that we are given points

$$
\left\{\begin{array}{l}
\Lambda_{1}^{0} \in \sqrt{-1} \check{\mathfrak{a}}_{1}, \\
\Lambda_{2}^{0} \in \sqrt{-1} \check{\mathfrak{a}}_{2} .
\end{array}\right.
$$

To indicate the dependence of our choices on $w_{12}$, fix $C^{\infty}$ functions

$$
\left\{\begin{array}{l}
\Lambda_{1}\left(w_{12}: ?\right): \sqrt{-1} \check{\mathfrak{a}} \rightarrow \sqrt{-1} \check{\mathfrak{a}}_{1}, \\
\Lambda_{2}\left(w_{12}: ?\right): \sqrt{-1} \check{\mathfrak{a}} \rightarrow \sqrt{-1} \check{\mathfrak{a}}_{2}
\end{array}\right.
$$

subject to:

(1) $\forall \Lambda \in \sqrt{-1} \check{\mathfrak{a}}: \Lambda_{1}\left(w_{12}: \Lambda\right)+w_{12} \bar{\Lambda}_{2}\left(w_{12}: \Lambda\right)=\Lambda$;

(2) $\exists \Lambda \in \sqrt{-1} \check{a}: \Lambda_{1}\left(w_{12}: \Lambda\right)=\Lambda_{1}^{0}$ and $\Lambda_{2}\left(w_{12}: \Lambda\right)=\Lambda_{2}^{0}$.

Observe that the $\Lambda$ figuring in (2) must be, by (1), $\Lambda_{1}^{0}+w_{12} \overline{\Lambda_{2}^{0}}$.

So, on the basis of $\S 10$ in $[2-(\mathbf{d})], \bmod o(\mathbf{H})$

$$
\left(Q^{\mathbf{H}} \mathbf{E}\left(P_{1} \mid A_{1}: \mathbf{\Phi}_{1}: \Lambda_{1}^{0}: ?\right), Q^{\mathbf{H}} \mathbf{E}\left(P_{2} \mid A_{2}: \Phi_{2}: \Lambda_{2}^{0}: ?\right)\right)
$$

equals

$$
(-1)^{l} \cdot \sum_{w_{12} \in W\left(A_{1}, A_{2}\right)} \mathbf{I I}_{\phi\left(w_{12}\right)}\left(\Lambda_{1}^{0}+w_{12} \overline{\Lambda_{2}^{0}} ; \mathbf{\Phi}_{1}, \boldsymbol{\Phi}_{2}: \mathbf{H}\right) .
$$

We stress that the $\mathbf{o}(\mathbf{H})$ does require careful, additional investigation but this can be put off for now.

Fix anew a $w_{12} \in W\left(A_{1}, A_{2}\right)$-then what has been said in $\S 4$ is applicable to the explicit evaluation of

$$
\text { III }_{\phi\left(w_{12}\right)}\left(\Lambda ; \boldsymbol{\Phi}_{1}, \boldsymbol{\Phi}_{2}: \mathbf{H}\right) \text {. }
$$

To formulate matters in just the right way, some preliminaries are needed. 
Fix a $\Gamma$-percuspidal $P_{0}$-then we can and will assume that the "base point" $P_{i} \in \mathscr{C}_{i}$ lies in the standard picture over $P_{0}$, i.e., $P_{i} \succeq P_{0}$. This is a technically helpful (and harmless) convention.

Let still $\mathscr{C}$ be an association class of $\Gamma$-cuspidal split parabolic subgroups of $G: \mathscr{C}=\bigsqcup_{i} \mathscr{C}_{i}$ the decomposition of $\mathscr{C}$ into $G$-conjugacy classes $\left(\mathscr{C}_{i}=G \cdot\left\{P_{i}\right\} \cap \mathscr{C}\right)$. Let $\mathscr{C}_{i^{\prime}}$ be some other $G$-conjugacy class relative to an association class $\mathscr{C}^{\prime}$ with the property that there is a $\mathscr{C}_{i}$ such that $\mathscr{C}_{i^{\prime}} \succeq \mathscr{C}_{i}$, thus $P_{i^{\prime}} \succeq P_{i}$. Of course, $\mathscr{C}_{i}$ is not necessarily unique, an irrelevancy as it turns out. Consider the associates $P_{j}$ of $P_{i}$ over $P_{0}$ lying below $P_{i^{\prime}}$-then $P_{j}^{\dagger}$ might or might not be associate to $P_{i}^{\dagger}$. Retaining only those $P_{j}$ for which the positive eventuality is true, the set of all $\Gamma$-cuspidals $G$-conjugate to some such $P_{j}$ is a $\Gamma$ stable subset $\mathscr{C}^{\dagger}\left(\mathscr{C}_{i^{\prime}}, \mathscr{C}_{i}\right)$ of $\mathscr{C}$, to be termed, somewhat imprecisely, a daggered association class.

EXAMPLE. In the set up of $\S 4$, take $V=\check{\mathfrak{a}}$-then a given $\Lambda \in \sqrt{-1} \check{\mathfrak{a}}$ determines a set $\mathscr{S}(\Lambda)$ of ordered triples $\left(\check{\mathfrak{a}}\left(F_{0}(\mathscr{C}: \Lambda)\right), \mathscr{C}_{\Lambda}, \mathscr{C}(\Lambda)\right)$. Owing to Lemma 4.1 , this set corresponds bijectively to

$$
\coprod_{i=1}^{r}\{i\} \times W\left(A_{i}, A\right) .
$$

Suppose that we freeze the first two entries of the triple but allow the third to vary. What does this mean in terms of the pairs $\left(i, w_{i}\right)$ ? The answer is basically contained in the descriptions to be found on pp. 66-70 of TES. Because the triple corresponds to a chamber and the chamber corresponds to a parabolic $P$ with split component $A$ admitting a domination $P_{\Lambda} \succeq P$ by a parabolic $P_{\Lambda}$ with a split component $A_{\Lambda}$ (per $\mathscr{C}_{\Lambda}$ ), the effect of changing $\mathscr{C}(\Lambda)$ is to change $P$ but not $P_{\Lambda}$. Passing to the standard picture over $P_{0}, P_{\Lambda}$ will be $G$-conjugate to a $\Gamma$-cuspidal $P_{i^{\prime}}$, so $\exists w_{\Lambda}^{0} \in W\left(A_{i^{\prime}}, A_{\Lambda}\right)$ taking $\mathscr{C}_{\Lambda}$ to its counterpart in $\check{\mathfrak{a}}_{i^{\prime}}$, while $P$ will be $G$-conjugate to a $\Gamma$-cuspidal $P_{i}$, the ambient $w_{i}$ agreeing with $w_{\Lambda}^{0}$ on $A_{\Lambda}$. Choose, accordingly, a $w_{i}^{0} \in W\left(A_{i}, A\right)$ such that $w_{i}^{0} \mid A_{\Lambda}=w_{\Lambda}^{0}$. Using the index $j$ to signify a variable $P$, the pairs $\left(j, w_{j}\right)$ arising from the $\mathscr{C}(\Lambda)$ are therefore the elements of the set

$$
\coprod_{j}\{j\} \times W_{\mathscr{E}_{\prime^{\prime}}}^{\dagger}\left(A_{j}, A_{i}\right) \cdot w_{i}^{0}
$$

Here (cf. pp. 130-131 in TES)

$$
W_{\mathscr{C}_{i^{\prime}}}^{\dagger}\left(A_{j}, A_{i}\right)=\left\{w_{j i} \in W\left(A_{j}, A_{i}\right): w_{j i} \mid A_{i^{\prime}}=\mathrm{ID}\right\}
$$


Finally, if we let $\mathscr{I}^{\dagger}\left(\mathscr{C}_{i^{\prime}}, \mathscr{C}_{i}\right)$ stand for the indices $j$ appearing in this decomposition, then the collection of all $\Gamma$-cuspidals $G$-conjugate to a $P_{j}\left(j \in \mathscr{I}^{\dagger}\left(\mathscr{C}_{i^{\prime}}, \mathscr{C}_{i}\right)\right)$ comprise a daggered association class $\mathscr{C}^{\dagger}\left(\mathscr{C}_{i^{\prime}}, \mathscr{C}_{i}\right)$.

To see how these considerations actually come up, let us place ourselves in the setting of Theorem 4.3 (bis). The role of the fixed vector $T$ is played by $\boldsymbol{\Phi}_{1} \otimes \boldsymbol{\Phi}_{2}$. Furthermore, the $T$-functions must be independent of $\mathscr{C}\left(\Lambda_{1}^{0}+w_{12} \overline{\Lambda_{2}^{0}}\right)$, hence dependent only on $\left(i, w_{i}^{0}\right)$. To be specific, given

$$
\left\{\begin{array}{l}
j \in \mathscr{I}^{\dagger}\left(\mathscr{C}_{i^{\prime}}, \mathscr{C}_{i}\right), \\
w_{j i} \in W_{\mathscr{C}_{\iota^{\prime}}}^{\dagger}\left(A_{j}, A_{i}\right),
\end{array}\right.
$$

call

$$
T\left(\left(j, w_{j i} w_{i}^{0}\right): w_{12}: \Lambda_{1}^{0}, \Lambda_{2}^{0}: \Lambda\right)
$$

the tensor product of

$$
\mathbf{c}\left(P_{i}\left|A_{i}: P_{1}\right| A_{1}: w_{i}^{0}: \Lambda_{1}\left(w_{12}: \Lambda\right)\right)^{-1} \cdot \mathbf{c}\left(P_{i}\left|A_{i}: P_{1}\right| A_{1}: w_{i}^{0}: \Lambda_{1}^{0}\right) \mathbf{\Phi}_{1}
$$

with

$$
\begin{aligned}
\mathbf{c}\left(P_{i}\left|A_{i}: P_{2}\right| A_{2}: w_{i}^{0} w_{12}: \Lambda_{2}\left(w_{12}: \Lambda\right)\right)^{-1} \\
\cdot \mathbf{c}\left(P_{i}\left|A_{i}: P_{2}\right| A_{2}: w_{i}^{0} w_{12}: \Lambda_{2}^{0}\right) \mathbf{\Phi}_{2} .
\end{aligned}
$$

Obviously,

$$
T\left(\left(j, w_{j i} w_{i}^{0}\right): w_{12}: \Lambda_{1}^{0}, \Lambda_{2}^{0}: \Lambda_{1}^{0}+w_{12} \overline{\Lambda_{2}^{0}}\right)=\boldsymbol{\Phi}_{1} \otimes \mathbf{\Phi}_{2} .
$$

Using the functional equations, we then find that

$$
\begin{array}{r}
\left\langle T\left(\left(j, w_{j i} w_{1}^{0}\right): w_{12}: \Lambda_{1}^{0}, \Lambda_{2}^{0}: \Lambda\right), \phi_{\left(j, w_{\jmath} w_{\imath}^{0}\right)}(\Lambda)\right\rangle \\
=\left\langle\mathbf{e}\left(\mathscr{C}_{j}: \mathbf{H}, w_{j i} w_{i}^{0} \Lambda\right),\left(1_{j i}(\Lambda), 2_{j i}(\Lambda)\right)_{\mathscr{C}_{\jmath}}\right\rangle,
\end{array}
$$

where

$$
\left\{\begin{aligned}
1_{j i}(\Lambda)= & \mathbf{c}\left(P_{j}\left|A_{j}: P_{i}\right| A_{i}: w_{j i}: w_{i}^{0} \Lambda_{1}\left(w_{12}: \Lambda\right)\right) \\
& \cdot \mathbf{c}\left(P_{i}\left|A_{i}\right| P_{1} \mid A_{1}: w_{i}^{0}: \Lambda_{1}^{0}\right) \mathbf{\Phi}_{1} \\
2_{j i}(\Lambda)= & \mathbf{c}\left(P_{j}\left|A_{j}: P_{i}\right| A_{i}: w_{j i}: w_{i}^{0} w_{12} \Lambda_{2}\left(w_{12}: \Lambda\right)\right) \\
& \cdot \mathbf{c}\left(P_{i}\left|A_{i}: P_{2}\right| A_{2}: w_{i}^{0} w_{12}: \Lambda_{2}^{0}\right) \mathbf{\Phi}_{2}
\end{aligned}\right.
$$

Applying now Theorem 4.3 (bis),

$$
\mathbf{I I}_{\phi\left(w_{12}\right)}\left(\Lambda_{1}^{0}+w_{12} \overline{\Lambda_{2}^{0}} ; \boldsymbol{\Phi}_{1}, \boldsymbol{\Phi}_{2}: \mathbf{H}\right)
$$

is the sum

$$
\sum_{\left(i, w_{\imath}^{0}\right)} \sum_{\left(j, w_{\jmath \prime}\right)}(-1)^{\#\left(F_{0}\left(\mathscr{C}\left(\Lambda_{1}^{0}+w_{12} \overline{\Lambda_{2}^{0}}\right)\right)\right)}
$$


of the

$$
\begin{aligned}
& D_{*}^{F_{0}\left(\mathscr{C}\left(\Lambda_{1}^{0}+w_{12} \overline{\Lambda_{2}^{0}}\right)\right)}\left[\frac{\left|D_{\mathscr{C}: \Lambda_{1}^{0}+w_{12} \overline{\Lambda_{2}^{0}}}\right|^{1 / 2}}{\left[\prod_{\lambda_{1} \in F_{0}(\mathscr{C})-F_{0}\left(\mathscr{E}\left(\Lambda_{1}^{0}+w_{12} \overline{\Lambda_{2}^{0}}\right)\right)}\left(?, \lambda_{i}\right)\right.}\right. \\
&\left.\quad \times\left\langle\mathbf{e}\left(\mathscr{C}_{j}: \mathbf{H}, w_{j i} w_{i}^{0} ?\right),\left(1_{j i}(?), 2_{j i}(?)\right)_{\mathscr{C}_{j}}\right\rangle\right]\left.\right|_{\Lambda_{1}^{0}+w_{12} \overline{\Lambda_{2}^{0}}} .
\end{aligned}
$$

We may interpret

$$
\left\{\begin{array}{l}
w_{j i} w_{i}^{0}\left(F_{0}(\mathscr{C})\right) \quad \text { as } \Sigma_{P_{J}}^{0}\left(\mathfrak{g}, \mathfrak{a}_{j}\right) \equiv \Sigma_{P_{J}}^{0} \\
w_{j i} w_{i}^{0}\left(F_{0}\left(\mathscr{C}\left(\Lambda_{1}^{0}+w_{12} \overline{\Lambda_{2}^{0}}\right)\right)\right) \text { as } \Sigma_{P_{J}^{\dagger}}^{0}\left(\mathfrak{m}_{j}, \mathfrak{a}_{j}^{\dagger}\right) \equiv \Sigma_{P_{J}^{\dagger}}^{0}
\end{array}\right.
$$

while

$$
\mid D_{\mathscr{C}: \Lambda_{1}^{0}+w_{12}}{\overline{\Lambda_{2}^{0}}}^{1 / 2}=\operatorname{vol}\left(\mathscr{C}_{i^{\prime}}\right)
$$

To complete the shift of burden, define

$$
\left\{\begin{array}{l}
\Lambda_{1}\left(w_{j i}, w_{12}: ?\right): \sqrt{-1} \check{\mathfrak{a}}_{j} \rightarrow \sqrt{-1} \check{\mathfrak{a}}_{1}, \\
\Lambda_{2}\left(w_{j i}, w_{12}: ?\right): \sqrt{-1} \check{\mathfrak{a}}_{j} \rightarrow \sqrt{-1} \check{\mathfrak{a}}_{2}
\end{array}\right.
$$

by

$$
\left\{\begin{array}{l}
\Lambda_{1}\left(w_{j i}, w_{12}: \Lambda_{j}\right)=\Lambda_{1}\left(w_{12}:\left(w_{j i} w_{i}^{0}\right)^{-1} \Lambda_{j}\right) \\
\Lambda_{2}\left(w_{j i}, w_{12}: \Lambda_{j}\right)=\Lambda_{2}\left(w_{12}:\left(w_{j i} w_{i}^{0}\right)^{-1} \Lambda_{j}\right)
\end{array}\right.
$$

Then

$$
\mathbf{I I}_{\phi\left(w_{12}\right)}\left(\Lambda_{1}^{0}+w_{12} \overline{\Lambda_{2}^{0}} ; \boldsymbol{\Phi}_{1}, \boldsymbol{\Phi}_{2}: \mathbf{H}\right)
$$

can be rewritten as the sum

$$
\sum_{\left(i, w_{\imath}^{0}\right)} \sum_{\left(j, w_{j l}\right)}
$$

of the

$$
\begin{aligned}
(-1)^{\#\left(\Sigma_{P_{j}^{\dagger}}^{0}\right)} \cdot D_{*}^{\Sigma_{P_{J}^{\dagger}}^{0}}\left[\frac{\operatorname{vol}\left(\mathscr{C}_{i^{\prime}}\right)}{\prod_{\lambda_{j} \in \Sigma_{P_{J}}^{0}-\Sigma_{P_{j}^{\dagger}}^{0}\left(?, \lambda_{j}\right)}}\right. \\
\left.\qquad \times\left\langle\mathbf{e}\left(\mathscr{C}_{j}: \mathbf{H}, ?\right),\left(1_{j i}(?), 2_{j i}(?)\right)_{\mathscr{C}_{J}}\right\rangle\right]\left.\right|_{w_{l l} w_{1}^{0}\left(\Lambda_{1}^{0}+w_{12} \overline{\Lambda_{2}^{0}}\right)},
\end{aligned}
$$

with the understanding that in the definitions of $1_{j i}$ and $2_{j i}$, the obvious replacements are to be made. 
ApPliCATION. Take, successively,

$$
\begin{aligned}
& \text { A : }\left\{\begin{array}{l}
\Lambda_{1}\left(w_{12}: \Lambda\right)=\Lambda-w_{12} \overline{\Lambda_{2}^{0}} \\
\Lambda_{2}\left(w_{12}: \Lambda\right)=\Lambda_{2}^{0}
\end{array} \quad(\Lambda \in \sqrt{-1} \check{\mathfrak{a}}),\right. \\
& \text { B : }\left\{\begin{array}{l}
\Lambda_{1}\left(w_{12}: \Lambda\right)=\Lambda_{1}^{0} \\
\Lambda_{2}\left(w_{12}: \Lambda\right)=w_{12}^{-1}\left(\bar{\Lambda}-\overline{\Lambda_{1}^{0}}\right)
\end{array} \quad(\Lambda \in \sqrt{-1} \check{\mathfrak{a}}),\right.
\end{aligned}
$$

to treat

$$
\Upsilon\left(\Lambda_{1}^{0}, \Lambda_{2}^{0} ; w_{12} ; \boldsymbol{\Phi}_{1}, \mathbf{\Phi}_{2}: \mathbf{H}\right)
$$

as

$$
\left\{\begin{array}{l}
\text { A : holomorphic in } \Lambda_{1}^{0}\left(\Lambda_{2}^{0} \text { fixed }\right) \\
\text { B : conjugate holomorphic in } \Lambda_{2}^{0}\left(\Lambda_{1}^{0} \text { fixed }\right) .
\end{array}\right.
$$

It then follows from Hartog's theorem that

$$
\left(\Lambda_{1}^{0}, \overline{\Lambda_{2}^{0}}\right) \mapsto \Upsilon\left(\Lambda_{1}^{0}, \Lambda_{2}^{0} ; w_{12} ; \boldsymbol{\Phi}_{1}, \mathbf{\Phi}_{2}: \mathbf{H}\right)
$$

is holomorphic on $\sqrt{-1} \check{\mathfrak{a}}_{1} \times \sqrt{-1} \check{\mathfrak{a}}_{2}$. We have therefore recovered the main conclusion in $\S 9$ of [2-(d)].

It will be convenient to retain the second choice above for $\Lambda_{1}\left(w_{12}: ?\right)$ and $\Lambda_{2}\left(w_{12}:\right.$ ?), i.e., B supra. In this situation, $1_{j i}$ is just a constant, namely

$$
\mathbf{c}\left(P_{j}\left|A_{j}: P_{1}\right| A_{1}: w_{j i} w_{i}^{0}: \Lambda_{1}^{0}\right) \mathbf{\Phi}_{1},
$$

while $2_{j i}$ becomes

$$
\begin{gathered}
\mathbf{c}\left(P_{i}\left|A_{i}: P_{j}\right| A_{j}: w_{j i}^{-1}: w_{j i} w_{i}^{0} \Lambda_{1}^{0}-\Lambda_{j}\right)^{*} \\
\quad \cdot \mathbf{c}\left(P_{i}\left|A_{i}: P_{2}\right| A_{2}: w_{i}^{0} w_{12}: \Lambda_{2}^{0}\right) \boldsymbol{\Phi}_{2} .
\end{gathered}
$$

Thus, we have proved:

Proposition 5.2. Fix

$$
\left\{\begin{array}{l}
\Lambda_{1}^{0} \in \sqrt{-1} \check{\mathfrak{a}}_{1}, \\
\Lambda_{2}^{0} \in \sqrt{-1} \check{\mathfrak{a}}_{2} .
\end{array}\right.
$$

Then $\bmod \mathrm{o}(\mathbf{H})$

$$
\left(Q^{\mathbf{H}} \mathbf{E}\left(P_{1} \mid A_{1}: \mathbf{\Phi}_{1}: \Lambda_{1}^{0}: ?\right), Q^{\mathbf{H}} \mathbf{E}\left(P_{2} \mid A_{2}: \boldsymbol{\Phi}_{2}: \Lambda_{2}^{0}: ?\right)\right)
$$

equals $(-1)^{l}$ times the sum over

$$
w_{12} \in w\left(A_{1}, A_{2}\right)
$$

of the sum

$$
\sum_{\left(i, w_{l}^{0}\right)} \sum_{\left(j, w_{\jmath \prime}\right)}
$$


of the

$$
\begin{aligned}
(-1)^{\#\left(\Sigma_{P_{j}^{\dagger}}^{0}\right)} \cdot D_{*}^{\Sigma_{P^{\dagger}}^{0}}\left[\frac{\operatorname{vol}\left(\mathscr{C}_{i^{\prime}}\right)}{\prod_{\lambda, \in \Sigma_{P_{J}}^{0}-\Sigma_{P_{j}^{\dagger}}^{0}\left(?, \lambda_{j}\right)}}\right. \\
\left.\qquad \times\left\langle\mathbf{e}\left(\mathscr{C}_{j}: \mathbf{H}, ?\right),\left(1_{j i}(?), 2_{j i}(?)\right)_{\mathscr{C}_{J}}\right\rangle\right]\left.\right|_{w_{j l} w_{l}^{0}\left(\Lambda_{1}^{0}+w_{12} \overline{\left.\Lambda_{2}^{0}\right)}\right.} .
\end{aligned}
$$

A relative version of this result will also be needed. To proceed in a manner amenable to calculation, we shall first have to deal with some representation-theoretic preliminaries, the subject of the next section.

6. Truncation of the induced representations. The purpose of this section is to discuss the truncation of the

$$
\operatorname{Ind}_{M \cdot A \cdot N}^{G}\left(L_{M / \Gamma_{M}} \otimes \Lambda \otimes 1\right) \quad(\Lambda \in \sqrt{-1} \check{a}) .
$$

Their introduction can be justified on the following grounds. We know from $\S 8$ of [2-(f)] that if $\alpha$ is a $K$-central, $K$-finite element of $C_{c}^{\infty}(G)$, then

$$
\left\{\begin{array}{l}
\operatorname{tr}\left(Q^{I_{M}(\mathbf{H})} \circ L_{M / \Gamma_{M}}^{\mathrm{dis}}\left(\alpha^{P}\right) \circ Q^{I_{M}(\mathbf{H})}\right) \\
\operatorname{tr}\left(Q^{I_{M}(\mathbf{H})} \circ L_{M / \Gamma_{M}}^{\operatorname{con}}\left(\alpha^{P}\right) \circ Q^{I_{M}(\mathbf{H})}\right)
\end{array}\right.
$$

are objects of fundamental importance. That being, the main result of the present section is an expression for these entities in terms of

$$
\left\{\begin{array}{l}
\int_{\operatorname{Re}(\Lambda)=0} \operatorname{tr}\left(Q_{P}^{\mathbf{H}} \circ \operatorname{Ind}_{M \cdot A \cdot N}^{G}\left(L_{M / \Gamma_{M}}^{\text {dis }} \otimes \Lambda \otimes 1\right)(\alpha) \circ Q_{P}^{\mathbf{H}}\right)|d \Lambda| \\
\int_{\operatorname{Re}(\Lambda)=0} \operatorname{tr}\left(Q_{P}^{\mathbf{H}} \circ \operatorname{Ind}_{M \cdot A \cdot N}^{G}\left(L_{M / \Gamma_{M}}^{\text {con }} \otimes \Lambda \otimes 1\right)(\alpha) \circ Q_{P}^{\mathbf{H}}\right)|d \Lambda| .
\end{array}\right.
$$

In turn, it will be shown that the integrands can be written as an integral-series involving inner products of "partial Eisenstein series", formulae for the latter following from an extension of Proposition 5.2 (cf. $\S 7)$.

Fix a $\Gamma$-cuspidal $P=M \cdot A \cdot N$-then, as in TES (pp. 95-96), attached to any $\Lambda \in \sqrt{-1} \check{a}$, there is a unitarily induced representation

$$
\operatorname{Ind}_{M \cdot A \cdot N}^{G}\left(L_{M / \Gamma_{M}} \otimes \Lambda \otimes 1\right)
$$

or still, by induction in stages,

$$
\operatorname{Ind}_{\Gamma_{M} \cdot A \cdot N}^{G}(1 \otimes \Lambda \otimes 1) .
$$

Methodically, it will be simplest to establish our results in the second picture although they will be explicitly formulated only in the first. The reason is that the representation space for

$$
\operatorname{Ind}_{\Gamma_{M} \cdot A \cdot N}^{G}(1 \otimes \Lambda \otimes 1)
$$


is tailored for a direct application of the partial truncation operator $Q_{P}^{\mathrm{H}}$ (cf. $\S 8$ in [2-(b)]) while, strictly speaking, this would not be true of the representation space for

$$
\operatorname{Ind}_{M \cdot A \cdot N}^{G}\left(L_{M / \Gamma_{M}} \otimes \Lambda \otimes 1\right) .
$$

Of course, no real difficulty is present, the issue being technical but minor. So, in particular, if $\alpha \in C_{c}^{\infty}(G)$, then

$$
Q_{P}^{\mathbf{H}} \circ \operatorname{Ind}_{M \cdot A \cdot N}^{G}\left(L_{M / \Gamma_{M}} \otimes \Lambda \otimes 1\right)(\alpha) \circ Q_{P}^{\mathbf{H}}
$$

makes sense through

$$
Q_{P}^{\mathrm{H}} \circ \operatorname{Ind}_{\Gamma_{M} \cdot A \cdot N}^{G}(1 \otimes \Lambda \otimes 1)(\alpha) \circ Q_{P}^{\mathrm{H}}
$$

and likewise in general (cf. infra).

Proposition 6.1. Let $\mathbf{H} \in \mathfrak{a}_{Q}$. Let $\alpha \in C_{c}^{\infty}(G)$ be $K$-finite-then $\forall \Lambda \in \sqrt{-1} \check{a}$,

$$
Q_{P}^{\mathbf{H}} \circ \operatorname{Ind}_{M \cdot A \cdot N}^{G}\left(L_{M / \Gamma_{M}} \otimes \Lambda \otimes 1\right)(\alpha) \circ Q_{P}^{\mathbf{H}}
$$

is of the trace class. Furthermore, $\forall$ natural $N, \exists C_{N}>0$ such that

$$
\tau\left(Q_{P}^{\mathbf{H}} \circ \operatorname{Ind}_{M \cdot A \cdot N}^{G}\left(L_{M / \Gamma_{M}} \otimes \Lambda \otimes 1\right)(\alpha) \circ Q_{P}^{\mathbf{H}}\right) \leq \frac{C_{N}}{(1+\|\Lambda\|)^{N}} .
$$

[Note: As usual, $\tau$ is the trace norm.]

It is therefore a corollary that

$$
\operatorname{tr}\left(Q_{P}^{\mathbf{H}} \circ \operatorname{Ind}_{M \cdot A \cdot N}^{G}\left(L_{M / \Gamma_{M}} \otimes \Lambda \otimes 1\right)(\alpha) \circ Q_{P}^{\mathbf{H}}\right)
$$

is a rapidly decreasing function on the imaginary axis.

Turning to the proof, recall that the representation space for

$$
\operatorname{Ind}_{\Gamma_{M} \cdot A \cdot N}^{G}(1 \otimes \Lambda \otimes 1)
$$

may be taken as $L^{2}(G /(\Gamma \cap P) \cdot A \cdot N)$ under the norm

$$
\|\Phi\|^{2}=\int_{K} \int_{M / \Gamma_{M}} \Phi(k m) \overline{\Phi(k m)} d_{K}(k) d_{M}(m),
$$

the action being

$$
\begin{aligned}
\operatorname{Ind}_{\Gamma_{M} \cdot A \cdot N}^{G} & (1 \otimes \Lambda \otimes 1)(x) \boldsymbol{\Phi}(y) \\
& =a_{x^{-1} y}^{(\Lambda-\rho)} \cdot a_{y}^{-(\Lambda-\rho)} \cdot \boldsymbol{\Phi}\left(x^{-1} y\right) \quad(x, y \in G) .
\end{aligned}
$$

It is well known and easy to show that

$$
\operatorname{Ind}_{\Gamma_{M} \cdot A \cdot n}^{G}(1 \otimes \Lambda \otimes 1)(\alpha)
$$


is an integral operator with kernel

$$
\begin{aligned}
& K_{\alpha}(P: x, y: \Lambda): \\
& \quad a_{x}^{(-\Lambda+\rho)} \cdot a_{y}^{(\Lambda+\rho)} \cdot \sum_{\delta \in \Gamma_{M}} \int_{A} \int_{N} \alpha\left(x \delta a n y^{-1}\right) a^{(-\Lambda+\rho)} d_{A}(a) d_{N}(n) .
\end{aligned}
$$

Substituting $P$ for $G$, what has been said in $\S 2$ of [2-(f)] then implies:

(1) $\forall \mathbf{H} \in \mathfrak{a}_{Q}$,

$$
Q_{P}^{\mathbf{H}} \circ \operatorname{Ind}_{\Gamma_{M} \cdot A \cdot N}^{G}(1 \otimes \Lambda \otimes 1)(\alpha) \circ Q_{P}^{\mathbf{H}}
$$

is represented by

$$
\left(Q_{P}^{\mathbf{H}}\right)_{(1)}\left(Q_{P}^{\mathbf{H}}\right)_{(2)} K_{\alpha}\left(P: ?_{1}, ?_{2}: \Lambda\right)
$$

(2) $\forall \mathbf{H} \in \mathfrak{a}_{Q}$, the operators

$$
\left\{\begin{array}{l}
Q_{P}^{\mathbf{H}} \circ \operatorname{Ind}_{\Gamma_{M} \cdot A \cdot N}^{G}(1 \otimes \Lambda \otimes 1)(\alpha), \\
\operatorname{Ind}_{\Gamma_{M} \cdot A \cdot N}^{G}(1 \otimes \Lambda \otimes 1)(\alpha) \circ Q_{P}^{\mathbf{H}}
\end{array}\right.
$$

are Hilbert-Schmidt;

(3) $\forall \mathbf{H} \in \mathfrak{a}_{Q}$, the operator

$$
Q_{P}^{\mathrm{H}} \circ \operatorname{Ind}_{\Gamma_{M} \cdot A \cdot N}^{G}(1 \otimes \Lambda \otimes 1)(\alpha) \circ Q_{P}^{\mathrm{H}}
$$

is of the trace class.

Consequently, the only really new aspect of Proposition 6.1 is the rapid decrease of

$$
\tau\left(Q_{P}^{\mathbf{H}} \circ \operatorname{Ind}_{\Gamma_{M} \circ A \circ N}^{G}(1 \otimes \Lambda \otimes 1)(\alpha) \circ Q_{P}^{\mathbf{H}}\right) \quad(\Lambda \in \sqrt{-1} \check{\mathfrak{a}}),
$$

which, when $\operatorname{rank}(\Gamma)=1$, is essentially in $\S 7$ of [2-(a)]. Thanks to the theory of the parametrix and the sublemma appearing on p. 43 of [2-(a)], it suffices, in general, to prove that $\forall$ natural $N$

$$
(1+\|\Lambda\|)^{N} \cdot\left\|Q_{P}^{\mathrm{H}} \circ \operatorname{Ind}_{\Gamma_{M} \cdot A \cdot N}^{G}(1 \otimes \Lambda \otimes 1)(\alpha)\right\|_{\mathrm{HS}} \quad(\Lambda \in \sqrt{-1} \mathfrak{\mathfrak { a }})
$$

stays bounded. For this purpose, begin by observing that

$$
\begin{aligned}
K_{\alpha} & \left(P: k_{1} m_{1}, k_{2} m_{2}: \Lambda\right) \\
& =\sum_{\delta \in \Gamma_{M}} \int_{A} \int_{N} \alpha\left(k_{1} m_{1} \delta a n m_{2}^{-1} k_{2}^{-1}\right) a^{(-\Lambda+\rho)} d_{A}(a) d_{N}(n) \\
& =\int_{A} a^{(-\Lambda+\rho)}\left(\sum_{\delta \in \Gamma_{M}} \int_{N} a\left(k_{1} m_{1} \delta a n m_{2}^{-1} k_{2}^{-1}\right) d_{N}(n)\right) d_{A}(a) .
\end{aligned}
$$


Next, extending the notation of $\S 8$ in [2-(f)], form

$$
\alpha^{P}\left(k_{1}: k_{2}: m: \alpha\right)=\int_{N} \alpha\left(k_{1} \operatorname{mank}_{2}^{-1}\right) d_{N}(n)
$$

so that

$$
\begin{aligned}
K_{\alpha} & \left(P: k_{1} m_{1}, k_{2} m_{2}: \Lambda\right) \\
\quad= & \int_{A} a^{(-\Lambda+\rho)}\left(\sum_{\delta \in \Gamma_{M}} \alpha^{P}\left(k_{1}: k_{2}: m_{1} \delta m_{2}^{-1}: a\right)\right) d_{A}(a) .
\end{aligned}
$$

Finally, to look at

$$
Q_{P}^{\mathrm{H}} \circ \operatorname{Ind}_{\Gamma_{M} \cdot A \cdot N}^{G}(1 \otimes \Lambda \otimes 1)(\alpha)
$$

amounts to studying

$$
\left(Q_{P}^{\mathbf{H}}\right)_{(1)} K_{\alpha}\left(P: ?_{1}, ?_{2}: \Lambda\right)
$$

or still, in an abuse of notation,

$$
\int_{A} a^{(-\Lambda+\rho)} Q_{(1)}^{I_{M}(\mathbf{H})}\left(\sum_{\delta \in \Gamma_{M}} \alpha^{P}\left(k_{1}: k_{2}: m_{1} \delta m_{2}^{-1}: a\right)\right) d_{A}(a) .
$$

As functions of $m \in M$ depending on a parameter $a \in A$, the $\alpha^{P}$ vanish identically if $a \notin C_{\alpha}, C_{\alpha}$ a certain compact subset of $A$. Easy variants on a standard theme then tell us that

$$
Q_{(1)}^{I_{M}(\mathbf{H})}\left(\sum_{\delta \in \Gamma_{M}} \alpha^{P}\left(k_{1}: k_{2}: m_{1} \delta m_{2}^{-1}: a\right)\right) \in R\left(M / \Gamma_{M}\right)
$$

uniformly after differentiation in $a \in A$. Take now a polynomial $p(\Lambda)$ and consider

$$
p(\Lambda) \cdot \int_{A} a^{(-\Lambda+\rho)} Q_{(1)}^{I_{M}(\mathbf{H})}\left(\sum_{\delta \in \Gamma_{M}} \alpha^{P}\left(k_{1}: k_{2}: m_{1} \delta m_{2}^{-1}: a\right)\right) d_{A}(a) .
$$

The $A$-integral is over a compact set. Moreover, $p(\Lambda)$ can be moved inside to reemerge as a derivative with respect to $a \in A$ :

$$
\partial_{p}\left(Q_{(1)}^{I_{M}(\mathbf{H})}\left(\sum_{\delta \in \Gamma_{M}} \alpha^{P}\left(k_{1}: k_{2}: m_{1} \delta m_{2}^{-1}: a\right)\right)\right)
$$

In turn, by the remarks above, this function is uniformly bounded in $k_{1}, k_{2}, m_{1}, m_{2}$. Our contention of decay thus follows immediately. 
[Note: If $p$ is any polynomial on $\sqrt{-1}$ ă then and

(1) $\partial_{p}\left(Q_{P}^{\mathbf{H}} \circ \operatorname{Ind}_{M \cdot A \cdot N}^{G}\left(L_{M / \Gamma_{M}} \otimes \Lambda \otimes 1\right)(\alpha) \circ Q_{P}^{\mathbf{H}}\right)$ is of the trace class

(2) $\forall$ natural $N, \exists C_{N}>0$ such that

$$
\tau\left(\partial_{p}\left(Q_{P}^{\mathbf{H}} \circ \operatorname{Ind}_{M \cdot A \cdot N}^{G}\left(L_{M / \Gamma_{M}} \otimes \Lambda \otimes 1\right)(\alpha) \circ Q_{P}^{\mathbf{H}}\right)\right) \leq \frac{C_{N}}{(1+\|\Lambda\|)^{N}} .
$$

In fact, employing the theory of the parametrix and taking into account Leibniz's rule, we see that it need only be shown that $\forall$ natural $N$

$$
(1+\|\Lambda\|)^{N} \cdot\left\|\partial_{p}\left(Q_{P}^{\mathrm{H}} \circ \operatorname{Ind}_{\Gamma_{M} \cdot A \cdot N}^{G}(1 \otimes \Lambda \otimes 1)(\alpha)\right)\right\|_{\mathrm{HS}} \quad(\Lambda \in \sqrt{-1} \check{\mathfrak{a}})
$$

stays bounded. This, however, is clear since differentiation by $p$ simply introduces the factor $p(\log a)$ into the $A$-integral, itself compactly supported on $C_{\alpha}$.]

Given an $M$-invariant subspace $\mathscr{H}$ of $L^{2}\left(M / \Gamma_{M}\right)$, we shall write $L_{M / \Gamma_{M}}^{\mathscr{L}}$ for the restriction of $L_{M / \Gamma_{M}}$ to $\mathscr{H}$. Reducing norms leads readily to:

Proposition 6.1 (bis). Let $\mathbf{H} \in \mathfrak{a}_{Q}$. Let $\alpha \in C_{c}^{\infty}(G)$ be K-finitethen $\forall \Lambda \in \sqrt{-1}$ ă

$$
Q_{P}^{\mathbf{H}} \circ \operatorname{Ind}_{M \cdot A \cdot N}^{G}\left(L_{M / \Gamma_{M}}^{\mathscr{P}} \otimes \Lambda \otimes 1\right)(\alpha) \circ Q_{P}^{\mathbf{H}}
$$

is of the trace class. Furthermore, $\forall$ natural $N, \exists C_{N}>0$ such that

$$
\tau\left(Q_{P}^{\mathbf{H}} \circ \operatorname{Ind}_{M \cdot A \cdot N}^{G}\left(L_{M / \Gamma_{M}}^{\mathscr{H}} \otimes \Lambda \otimes 1\right)(\alpha) \circ Q_{P}^{\mathbf{H}}\right) \leq \frac{C_{N}}{(1+\|\Lambda\|)^{N}} .
$$

Accordingly,

$$
\operatorname{tr}\left(Q_{P}^{\mathbf{H}} \circ \operatorname{Ind}_{M \cdot A \cdot N}^{G}\left(L_{M / \Gamma_{M}}^{\mathscr{H}} \otimes \Lambda \otimes 1\right)(\alpha) \circ Q_{P}^{\mathbf{H}}\right)
$$

is a rapidly decreasing function on the imaginary axis, hence is integrable there. In a word, our objective will be to identify this integral, at least when $\mathscr{H}$ satisfies some supplementary conditions.

Apart from tacitly imposing the customary assumptions per $\mathscr{H}$ relative to the $K_{M}$-finite elements of $C_{c}^{\infty}(M)$ vis-à-vis the associated kernels (see $\S 2$ of [2-(f)]), we shall explicitly insist on the following condition:

$(\mathscr{H})$ Let $P_{I}^{\mathscr{H}}$ be the orthogonal projection of $L^{2}(G /(\Gamma \cap P) \cdot A \cdot N)$ onto

$$
L^{2}(G /(\Gamma \cap P) \cdot A \cdot N: \mathscr{H})
$$


the subspace made up of the $\Phi$ such that for every $x \in G$, the function

$$
m \mapsto \Phi(x m) \text { belongs to } \mathscr{H} \text {. }
$$

Given a $K$-finite element $\alpha$ of $C_{c}^{\infty}(G)$, it will be assumed that $\forall \Lambda \in$ $\sqrt{-1} \check{a}$,

$$
P_{I}^{\mathscr{H}} \circ \operatorname{Ind}_{\Gamma_{M} \cdot A \cdot N}^{G}(1 \otimes \Lambda \otimes 1)(\alpha) \circ P_{I}^{\mathscr{H}}
$$

is an integral operator admitting a kernel

$$
K_{\alpha}(P: \mathscr{H}: x, y: \Lambda) \in S_{r}^{\infty}(G / \Gamma \times G / \Gamma),
$$

where $r$ is independent of $\alpha$.

[Note: $P_{I}^{\mathscr{H}}$ is not to be confused with $P^{\mathscr{H}}$, the orthogonal projection of $L^{2}\left(M / \Gamma_{M}\right)$ onto $\mathscr{H}$. The connection between the two can be found in the commutative diagram

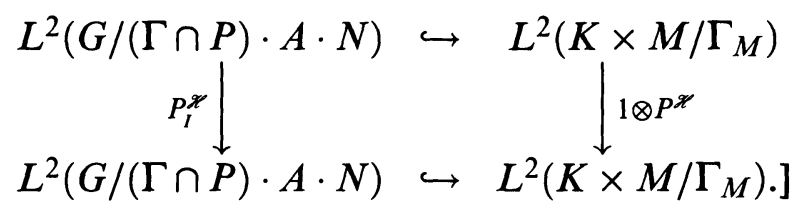

TheOREM 6.2. Let $\mathbf{H} \in \mathfrak{a}_{Q}$. Let $\alpha \in C_{\mathcal{c}}^{\infty}(G)$ be $K$-central and $K$ finite-then, under condition ( $\mathscr{H})$,

$$
\frac{1}{(2 \pi)^{l}} \int_{\operatorname{Re}(\Lambda)=0} \operatorname{tr}\left(Q_{P}^{\mathbf{H}} \circ \operatorname{Ind}_{M \cdot A \cdot N}^{G}\left(L_{M / \Gamma_{M}}^{\mathscr{H}} \otimes \Lambda \otimes 1\right)(\alpha) \circ Q_{P}^{\mathbf{H}}\right)|d \Lambda|
$$

equals

$$
\operatorname{tr}\left(Q^{I_{M}(\mathbf{H})} \circ L_{M / \Gamma_{M}}^{\mathscr{H}}\left(\alpha^{P}\right) \circ Q^{I_{M}(\mathbf{H})}\right)
$$

For orientation, we remark that if $\mathscr{H}=L_{\text {dis }}^{2}\left(M / \Gamma_{M}\right)$ or $\mathscr{H}=$ $L_{\text {con }}^{2}\left(M / \Gamma_{M}\right)$, then condition $(\mathscr{H})$ is necessarily in force (cf. infra) while, automatically, this is the case when $\mathscr{H}=L^{2}\left(M / \Gamma_{M}\right)$ itself.

Formally, the truth of the theorem is seemingly apparent. To proceed rigorously, however, is quite a different matter. The lemma below is the key to our method.

LEMMA 6.3. Let $X$ and $Y$ be second countable, locally compact Hausdorff spaces. Let

$$
\left\{\begin{array}{l}
\mu \text { be a regular Borel measure on } X \text { st } \operatorname{spt}(\mu)=X, \\
\nu \text { be a regular Borel measure on } Y .
\end{array}\right.
$$

Suppose that $K_{1}$ and $K_{2}$ are continuous functions on $X \times Y$ and that $T$ is a bounded linear transformation $L^{2}(Y ; \nu) \rightarrow L^{2}(Y ; \nu)$, all connected 
by:

(i) $\forall x \in X, K_{1}(x, ?) \in L^{2}(Y ; \nu)$ and $\forall$ compact $\Omega \subset X, \exists g_{\Omega} \in$ $L^{2}(Y ; \nu)$ such that $\forall x \in \Omega$,

$$
\left|K_{1}(x, y)\right| \leq g_{\Omega}(y) \quad \forall \text { a.e. } y \in Y ;
$$

(ii) $\forall g \in L_{c}^{2}(Y ; \nu)$,

$$
\int_{Y} K_{1}(x, y)(T g)(y) d \nu(y)=\int_{Y} K_{2}(x, y) g(y) d \nu(y) \quad \forall \text { a.e. } x \in X \text {. }
$$

Then the map

$$
g \mapsto \int_{Y} K_{1}(?, y) g(y) d \nu(y)
$$

takes $L^{2}(Y ; \nu)$ continuously to $C(X)$. Moreover, $\forall x \in X$,

$$
K_{2}(x, ?) \in L^{2}(Y ; \nu)
$$

and

$$
K_{2}(x, y)=\overline{\left(T^{*} \overline{K_{1}(x, ?)}\right)(y)} \quad \forall \text { a.e. } y \in Y .
$$

[Note: Recall that a second countable, locally compact Hausdorff space is necessarily sigma-compact and metrizable.]

Proof. Let $g \in L^{2}(Y ; \nu)$-then, by (i) $\forall x \in X, K_{1}(x, ?) \in L^{2}(Y ; \nu)$, thus

$$
\int_{Y} K_{1}(x, y) g(y) d \nu(y)
$$

is meaningful. To ascertain its continuity in $x$, assume that $\left\{x_{n}: n \in\right.$ $\mathbf{N}\}$ is a sequence in $X$ with limit $x$. Because $\left\{x_{n}: n \in \mathbf{N}\right\} \cup\{x\}$ is compact, in view of (i) again, there exists a $g_{0} \in L^{2}(Y ; \nu)$ such that

$$
\left\{\begin{array}{l}
\left|K_{1}\left(x_{n}, y\right)\right| \\
\left|K_{1}(x, y)\right|
\end{array} \leq\left|g_{0}(y)\right| \forall \text { a.e. } y \in Y .\right.
$$

So, up to a set of $\nu$-measure zero,

$$
\left\{\begin{array}{l}
\left|K_{1}\left(x_{n}, y\right)\right| \cdot|g(y)| \\
\left|K_{1}(x, y)\right| \cdot|g(y)|
\end{array} \leq\left|g_{0}(y)\right| \cdot|g(y)| \in L^{1}(Y ; \nu),\right.
$$

from which, by dominated convergence,

$$
\lim _{n \rightarrow \infty} \int_{Y} K_{1}\left(x_{n}, y\right) g(y) d \nu(y)=\int_{Y} K_{1}(x, y) g(y) d \nu(y) .
$$

Therefore

$$
\int_{Y} K_{1}(x, y) g(y) d \nu(y)
$$


is a continuous function of $x$. Moreover, if $\Omega \subset X$ is any compactum, then $\forall \varepsilon>0$,

$$
\|g\|_{2} \leq \varepsilon /\left\|g_{\Omega}\right\| \Rightarrow \sup _{x \in \Omega}\left|\int_{Y} K_{1}(x, y) g(y) d \nu(y)\right| \leq \varepsilon .
$$

By continuity and the fact that $\operatorname{spt}(\mu)=X$, we now see that the relation postulated by (ii) actually must hold for all $x$. That being, fix an $x-$ then, for any $g \in L_{c}^{2}(Y ; \nu)$,

$$
\begin{aligned}
\int_{Y} K_{2}(x, y) g(y) d \nu(y)=\int_{Y} K_{1}(x, y)(T g)(y) d \nu(y) \\
\quad=\left(T g, \overline{K_{1}(x, ?)}\right)=\left(g, T^{*} \overline{K_{1}(x, ?)}\right) \\
\quad=\int_{Y} \overline{\left(T^{*} \overline{K_{1}(x, ?)}\right)(y)} g(y) d \nu(y) .
\end{aligned}
$$

This implies that

$$
K_{2}(x, y)=\overline{\left(T^{*} \overline{K_{1}(x, ?)}\right)(y)}
$$

$\forall$ a.e. $y$ in an arbitrary relatively compact, open subset of $Y$, thence, by $\sigma$-compactness, $\forall$ a.e. $y \in Y$, period. As the right-hand side is in $L^{2}(Y ; \nu)$, the same is true of the left-hand side.

REMARK. It is a simple consequence of the foregoing argument that $\forall g \in L^{2}(Y ; \nu)$,

$$
\int_{Y} K_{1}(x, y)(T g)(y) d \nu(y)=\int_{Y} K_{2}(x, y) g(y) d \nu(y) \quad \forall x \in X .
$$

We omit the half dozen lines of justification.

Here is an initial application of the lemma. Take

$$
\left\{\begin{array}{l}
X=G /(\Gamma \cap P) \cdot A \cdot N, \\
Y=G /(\Gamma \cap P) \cdot A \cdot N
\end{array}\right.
$$

and let $T=P_{I}^{\mathscr{H}}$-then, in the presence of condition $(\mathscr{H})$, with

$$
\left\{\begin{array}{l}
K_{1}(x, y)=K_{\alpha}(P: x, y: \Lambda), \\
K_{2}(x, y)=K_{\alpha}(P: \mathscr{H}: x, y: \Lambda),
\end{array}\right.
$$

all the assumptions are met. In particular, we deduce that $\forall x \in X$,

$$
K_{\alpha}(P: \mathscr{H}: x, y: \Lambda)=\overline{P_{I}^{\mathscr{H}} \overline{K_{\alpha}(P: x, ?: \Lambda)}(y)} \quad \forall \text { a.e. } y \in Y \text {. }
$$

The operator

$$
Q_{P}^{\mathbf{H}} \circ \operatorname{Ind}_{M \cdot A \cdot N}^{G}\left(L_{M / \Gamma_{M}}^{\mathscr{H}} \otimes \Lambda \otimes 1\right)(\alpha) \circ Q_{P}^{\mathbf{H}}
$$


is represented, after transfer of models, by

$$
\left(Q_{P}^{\mathbf{H}}\right)_{(1)}\left(Q_{P}^{\mathbf{H}}\right)_{(2)} K_{\alpha}\left(P: \mathscr{H}: ?_{1}, ?_{2}: \Lambda\right) .
$$

In addition,

$$
\operatorname{tr}\left(Q_{P}^{\mathbf{H}} \circ \operatorname{Ind}_{M \cdot A \cdot N}^{G}\left(L_{M / \Gamma_{M}}^{\mathscr{H}} \otimes \Lambda \otimes 1\right)(\alpha) \circ Q_{P}^{\mathbf{H}}\right)
$$

can be calculated as the integral

$$
\int_{K} \int_{M / \Gamma_{M}} Q_{(1)}^{I_{M}(\mathbf{H})} Q_{(2)}^{I_{M}(\mathbf{H})} K_{\alpha}(P: \mathscr{H}: k m, k m: \Lambda) d_{K}(k) d_{M}(m) .
$$

Obviously, then, Theorem 6.2 hinges upon an examination of the latter integrand.

Put

$$
\left\{\begin{array}{l}
K_{\alpha}\left(P: k_{1}, m_{1}: k_{2}, m_{2}: \Lambda\right)=K_{\alpha}\left(P: k_{1} m_{1}: k_{2} m_{2}: \Lambda\right), \\
K_{\alpha}\left(P: \mathscr{H}: k_{1}, m_{1}: k_{2}, m_{2}: \Lambda\right)=K_{\alpha}\left(P: \mathscr{H}: k_{1} m_{1}, k_{2} m_{2}: \Lambda\right) .
\end{array}\right.
$$

Then

$$
K_{\alpha}\left(P: \mathscr{H}: k_{1}, m_{1}: ?, ?: \Lambda\right)=\left(1 \otimes P^{\mathscr{H}}\right) K_{\alpha}\left(P: k_{1}, m_{1}: ?, ?: \Lambda\right)
$$

for all $k_{1}, m_{1}$, which, being an $L^{2}$-equality, gives $\forall$ a.e. $k_{2} \in K$,

$$
\begin{aligned}
& K_{\alpha}\left(P: \mathscr{H}: k_{1}, m_{1}: k_{2}, m_{2}: \Lambda\right) \\
& \quad=P^{\mathscr{H}} K_{\alpha}\left(P: k_{1}, m_{1}: k_{2}, ?: \Lambda\right)\left(m_{2}\right) \quad \forall \text { a.e. } m_{2} \in M .
\end{aligned}
$$

This inference can be strengthened by taking

$$
\left\{\begin{array}{l}
X=K, \\
Y=M / \Gamma_{M}, \quad T=P^{\mathscr{H}},
\end{array}\right.
$$

and using the lemma, to wit: $\forall k_{1}, k_{2} \in K, \forall m_{1} \in M_{1}$,

$$
\begin{aligned}
K_{\alpha}(P & \left.: \mathscr{H}: k_{1}, m_{1}: k_{2}, m_{2}: \Lambda\right) \\
& =\overline{K_{\alpha^{*}}\left(P: \mathscr{H}: k_{2}, m_{2}: k_{1}, m_{1}: \Lambda\right)} \\
& =P_{\mathscr{H}} \overline{K_{\alpha^{*}}\left(P: k_{2}, ?: k_{1}, m_{1}: \Lambda\right)}\left(m_{2}\right) \\
& =P_{\mathscr{H}} K_{\alpha}\left(P: k_{1}, m_{1}: k_{2}, ?: \Lambda\right)\left(m_{2}\right) \quad \forall \text { a.e. } m_{2} \in M .
\end{aligned}
$$

Fix an $a \in A$. Let

$$
\Delta_{\alpha}\left(P: k_{1}, m_{1}: k_{2}, m_{2}: a\right)=\sum_{\delta \in \Gamma_{M}} \alpha^{P}\left(k_{1}: k_{2}: m_{1} \delta m_{2}^{-1}: a\right) .
$$

In this notation,

$$
K_{\alpha}\left(P: k_{1} m_{1}, k_{2} m_{2}: \Lambda\right)
$$

or still

$$
K_{\alpha}\left(P: k_{1}, m_{1}: k_{2}, m_{2}: \Lambda\right)
$$


is given by

$$
\int_{A} a^{(-\Lambda+\rho)} \Delta_{\alpha}\left(P: k_{1}, m_{1}: k_{2}, m_{2}: a\right) d_{A}(a) \text {. }
$$

Freeze $k_{1}, m_{1}$, and $k_{2}$-then, as has been noted earlier, the integral is uniformly compactly supported. Furthermore, the $\Delta_{\alpha}(P: \ldots)$, qua functions of $m_{2}$, are uniformly bounded. In light of this, there is a map

$$
A \rightarrow L^{2}\left(M / \Gamma_{M}\right)
$$

namely the rule assigning to each $a \in A$ the function

$$
\Delta_{\alpha}\left(P: k_{1}, m_{1}: k_{2}, ?: a\right) \text {. }
$$

It is clear that for every $f \in L^{2}\left(M / \Gamma_{M}\right)$,

$$
\begin{aligned}
& \int_{M / \Gamma_{M}} K_{\alpha}\left(P: \mathscr{H}: k_{1}, m_{1}: k_{2}, m_{2}: \Lambda\right) f\left(m_{2}\right) d_{M}\left(m_{2}\right) \\
&= \int_{M / \Gamma_{M}} P^{\mathscr{H}} K_{\alpha}\left(P: k_{1}, m_{1}: k_{2}, ?: \Lambda\right)\left(m_{2}\right) f\left(m_{2}\right) d_{M}\left(m_{2}\right) \\
&= \int_{M / \Gamma_{M}}\left((w) \int_{A} a^{(-\Lambda+\rho)} \overline{P^{\mathscr{H}} \overline{\Delta_{\alpha}\left(P: k_{1}, m_{1}: k_{2}, ?: a\right)}} d_{A}(a)\right)\left(m_{2}\right) \\
& \cdot f\left(m_{2}\right) d_{M}\left(m_{2}\right),
\end{aligned}
$$

$(w)$ signifying weak integral. Consequently,

$$
\begin{aligned}
K_{\alpha} & \left(P: \mathscr{H}: k_{1}, m_{1}: k_{2}, ?: \Lambda\right) \\
\quad & (w) \int_{A} a^{(-\Lambda+\rho)} \overline{P^{\mathscr{H}} \overline{\Delta_{\alpha}\left(P: k_{1}, m_{1}: k_{1}, ? ; a\right)}} d_{A}(a)
\end{aligned}
$$

as square integrable functions on $M / \Gamma_{M}$.

To exploit joint differentiability and thereby eliminate the sets of measure zero from the preceding expression, observe that for fixed $k_{1}$, $k_{2}$, and $a$,

$$
\Delta_{\alpha}\left(P: k_{1}, m_{1}: k_{2}, m_{2}: a\right)
$$

is the kernel of

$$
L_{M / \Gamma_{M}}\left(\alpha^{P}\left(k_{1}: k_{2}: ?: a\right)\right)
$$

at $m_{1}, m_{2}$. Invoking the lemma one last time with

$$
\left\{\begin{array}{l}
X=M / \Gamma_{M}, \\
Y=M / \Gamma_{M},
\end{array} \quad T=P^{\mathscr{H}},\right.
$$

and recalling our standing (tacit) hypothesis on $\mathscr{H}$, we find that $K_{\alpha^{P}\left(k_{1}: k_{2}: ?: a\right)}^{\mathscr{B}}$ belongs to the function class determined by

$$
\overline{P^{\mathscr{K}} \overline{\Delta_{\alpha}\left(P: k_{1}, ?: k_{2}, ?: a\right)}} \text {. }
$$


It therefore follows that $\forall k_{1}, k_{2} \in K, \forall m_{1}, m_{2} \in M$,

$$
\begin{aligned}
& K_{\alpha}\left(P: \mathscr{H}: k_{1}, m_{1}: k_{2}, m_{2}: \Lambda\right) \\
& \quad=\int_{A} a^{(-\Lambda+\rho)} K_{\alpha^{P}\left(k_{1}: k_{2}: ?: a\right)}^{\mathscr{P}}\left(m_{1}, m_{2}\right) d_{A}(a) .
\end{aligned}
$$

To complete the proof of Theorem 6.2, come back to

$$
\operatorname{tr}\left(Q_{P}^{\mathbf{H}} \circ \operatorname{Ind}_{M \cdot A \cdot N}^{G}\left(L_{M / \Gamma_{M}}^{\mathscr{H}} \otimes \Lambda \otimes 1\right)(\alpha) \circ Q_{P}^{\mathbf{H}}\right),
$$

that is, to

$$
\int_{K} \int_{M / \Gamma_{M}} Q_{(1)}^{I_{M}(\mathbf{H})} Q_{(2)}^{I_{M}(\mathbf{H})} K_{\alpha}(P: \mathscr{H}: k m, k m: \Lambda) d_{K}(k) d_{M}(m)
$$

or still, by the above

$$
\int_{K} \int_{A} a^{(-\Lambda+\rho)}\left[\int_{M / \Gamma_{M}} Q_{(1)}^{I_{M}(\mathbf{H})} Q_{(2)}^{I_{M}(\mathbf{H})} K_{\alpha^{P}(k: k: ?: a)}^{\mathscr{R}}(m, m) d_{M}(m)\right]_{\cdot d_{K}(k) d_{A}(a) .}
$$

Thought of as a function of $a \in A$,

$$
\int_{M / \Gamma_{M}} Q_{(1)}^{I_{M}(\mathbf{H})} Q_{(2)}^{I_{M}(\mathbf{H})} K_{\alpha^{P}(k: k: ?: a)}^{\mathscr{L}}(m, m) d_{M}(m)
$$

belongs to $C_{c}^{\infty}(A)$; on the other hand, of course,

$$
\begin{aligned}
\int_{M / \Gamma_{M}} & Q_{(1)}^{I_{M}(\mathbf{H})} Q_{(2)}^{I_{M}(\mathbf{H})} K_{\alpha^{P}(k: k: ?: a)}^{\mathscr{L}}(m, m) d_{M}(m) \\
& =\operatorname{tr}\left(Q^{I_{M}(\mathbf{H})} \circ L_{M / \Gamma_{M}}^{\mathscr{H}}\left(\alpha^{P}(k: k: ?: a)\right) \circ Q^{I_{M}(\mathbf{H})}\right) .
\end{aligned}
$$

Owing to the Fourier inversion formula, we now have

$$
\begin{gathered}
\frac{1}{(2 \pi)^{l}} \cdot \int_{\operatorname{Re}(\Lambda)=0} \operatorname{tr}\left(Q_{P}^{\mathbf{H}} \circ \operatorname{Ind}_{M \cdot A \cdot N}^{G}\left(L_{M / \Gamma_{M}}^{\mathscr{L}} \otimes \Lambda \otimes 1\right)(\alpha) \circ Q_{P}^{\mathbf{H}}\right)|d \Lambda| \\
\quad=\int_{K} \operatorname{tr}\left(Q^{I_{M}(\mathbf{H})} \circ L_{M / \Gamma_{M}}^{\mathscr{H}}\left(\alpha^{P}(k: k: ?: 1)\right) \circ Q^{I_{M}(\mathbf{H})}\right) d_{K}(k) .
\end{gathered}
$$

Since $\alpha$ is $K$-central, $\forall m \in M$,

$$
\begin{aligned}
\alpha^{P}(k: k: m: 1) & =\int_{N} \alpha\left(k m n k^{-1}\right) d_{N}(n) \\
& =\int_{N} \alpha(m n) d_{N}(n)=\alpha^{P}(m)
\end{aligned}
$$

in the notation of $\S 8$ in [2-(f)]. The integral over $K$ thus reduces to

$$
\operatorname{tr}\left(Q^{I_{M}(\mathbf{H})} \circ L_{M / \Gamma_{M}}^{\mathscr{H}}\left(\alpha^{P}\right) \circ Q^{I_{M}(\mathbf{H})}\right),
$$

the sought for conclusion. 
Having taken care of the proof of the theorem, we should say a word or two as to why condition $(\mathscr{H})$ holds if $\mathscr{H}=L_{\mathrm{dis}}^{2}\left(M / \Gamma_{M}\right)$ or $\mathscr{H}=L_{\text {con }}^{2}\left(M / \Gamma_{M}\right)$. Basically, it is simply a matter of "relativizing" the results in Chapter 8 of TES, i.e., of replacing $G$ by $P$ in their formulations and proofs. Let us give a brief indication of what is involved.

Suppose that

$$
P=M \cdot A \cdot N \succ P_{i}=M_{i} \cdot A_{i} \cdot N_{i} .
$$

Let $\boldsymbol{\Phi}_{i} \in \mathscr{E}\left(\delta, \mathscr{O}_{i}\right)$-then, using daggered Eisenstein system functions, the lemma on p. 300 of TES says that the series

$$
\sum_{\gamma \in \Gamma \cap P / \Gamma \cap P_{i}} a_{i}(x \gamma)^{\left(\Lambda_{i}^{\dagger}-\rho_{\imath}^{\dagger}\right)} \cdot \boldsymbol{\Phi}_{i}(x \gamma)
$$

can be meromorphically continued as a function of $\Lambda_{i}^{\dagger}$ from

$$
\mathscr{T}_{P_{i}^{\dagger}}\left(\check{\mathfrak{a}}_{i}^{\dagger}\right)+\sqrt{-1} \check{\mathfrak{a}}_{i}^{\dagger} \text { to } \check{\mathfrak{a}}_{i}^{\dagger}+\sqrt{-1} \check{\mathfrak{a}}_{i}^{\dagger} .
$$

We shall denote the meromorphic function thus obtained by the symbol

$$
E\left(P\left|A: P_{i}\right| A_{i}: \boldsymbol{\Phi}_{i}: \Lambda_{i}^{\dagger}: x\right)
$$

and refer to it as a "partial" Eisenstein series. Of course, if $P=G$, then it is a question of an Eisenstein series in the usual sense of the word. Finally, to reflect the decomposition

$$
\Lambda_{i}=\Lambda_{i}^{\dagger}+\Lambda
$$

it will be convenient to set

$$
\begin{aligned}
& E\left(P\left|A: P_{i}\right| A_{i}: \boldsymbol{\Phi}_{i}: \Lambda_{i}: x\right) \\
& \quad=a_{x}^{(\Lambda-\rho)} \cdot E\left(P\left|A: P_{i}\right| A_{i}: \boldsymbol{\Phi}_{i}: \Lambda_{i}^{\dagger}: x\right),
\end{aligned}
$$

legitimate since

$$
\begin{aligned}
& \sum_{\gamma \in \Gamma \cap P / \Gamma \cap P_{\imath}} a_{i}(x \gamma)^{\left(\Lambda_{i}-\rho_{i}\right)} \cdot \boldsymbol{\Phi}_{i}(x \gamma) \\
& =a_{x}^{(\Lambda-\rho)} \cdot \sum_{\gamma \in \Gamma \cap P / \Gamma \cap P_{i}} a_{i}(x \gamma)^{\left(\Lambda_{\iota}^{\dagger}-\rho_{\imath}^{\dagger}\right)} \cdot \boldsymbol{\Phi}_{i}(x \gamma) .
\end{aligned}
$$

With this preparation, we are now in a position to write down formulas for

$$
K_{\alpha}(P: \mathscr{H}: x, y: \Lambda) \quad\left\{\begin{array}{l}
\mathscr{H}=L_{\text {dis }}^{2}\left(M / \Gamma_{M}\right) \\
\mathscr{H}=L_{\text {con }}^{2}\left(M / \Gamma_{M}\right)
\end{array}\right.
$$


interest, for the most part, centering on the continuous case.

Fix an association class $\mathscr{C} \neq\{G\}$ :

$$
\mathscr{C}=\coprod_{i=1}^{r} \mathscr{C}_{i},
$$

where $\mathscr{C}_{i}=G \cdot\left\{P_{i}\right\} \cap \mathscr{C}$. Recalling that $P_{i \mu}\left(1 \leq \mu \leq r_{i}\right)$ is a set of representatives for $\Gamma \backslash \mathscr{C}_{i}$, we can and will assume that our orthonormal basis $\left\{\mathbf{e}_{n}^{i}: n \in I\left(\mathscr{\theta}_{i}\right)\right\}$ has been so selected that each $\mathbf{e}_{n}^{i}$ has but one nonzero component, thus lives in some $\mathscr{E}\left(\delta, \mathscr{O}_{i \mu}\right)$. In addition, we shall agree to let

$$
\operatorname{Dom}(P: \mathscr{C})=\left\{\mathscr{C}_{i}: P \succ \mathscr{C}_{i}\right\}
$$

If $\operatorname{Dom}(P: \mathscr{C})$ is not empty, then, per a particular $\mathscr{C}_{i}$ dominated by $P$, there is at least one $\mu$ for which $P_{i \mu}$ has a $\Gamma$-conjugate below $P$, say

$$
P \succ \gamma_{i \mu} P_{i \mu} \gamma_{i \mu}^{-1} \quad\left(\gamma_{i \mu} \in \Gamma\right) \text {. }
$$

Relative to an $\mathrm{e}_{n}^{i}$ singled out by such a $\mu$, put

$\mathbf{E}\left(P\left|A: P_{i}\right| A_{i}: \mathbf{e}_{n}^{i}: \Lambda_{i}: x\right)$

$$
=E\left(P\left|A: \gamma_{i \mu} P_{i \mu} \gamma_{i \mu}^{-1}\right| \gamma_{i \mu} A_{i \mu} \gamma_{i \mu}^{-1}: \Omega_{\gamma_{l \mu}}\left(k_{i \mu} \cdot \Lambda_{i}\right)\left(\mathbf{e}_{n}^{i}\right)_{\mu}: \gamma_{i \mu} k_{i \mu} \cdot \Lambda_{i}: x\right) .
$$

Lastly, choose a $k_{i} \in K$ with the property that

$$
k_{i} P k_{i}^{-1} \succ P_{i} \text {. }
$$

The notation established,

$$
K_{\alpha}\left(P: L_{\mathrm{con}}^{2}\left(M / \Gamma_{M}\right): x, y: \Lambda\right)
$$

is given by the sum

$$
\sum_{\mathscr{C}} \sum_{i: \mathscr{C}_{i} \in \operatorname{Dom}(P: \mathscr{C})} \sum_{\mathscr{G}_{i}}
$$

of

$$
\frac{1}{(2 \pi)^{l^{\dagger}}} \cdot \frac{1}{*\left(\mathscr{C}_{i}^{\dagger}\right)}
$$

times the integral

$$
\int_{\operatorname{Re}\left(\Lambda_{i}^{\dagger}\right)=0}
$$

of

$\sum_{m, n}^{(P)} \mathbf{C}_{m n}\left(\alpha: \boldsymbol{\theta}_{i}: k_{i} \cdot \Lambda+\Lambda_{i}^{\dagger}\right)$

$\times \mathbf{E}\left(P\left|A: P_{i}\right| A_{i}: \mathbf{e}_{m}^{i}: k_{i} \cdot \Lambda+\Lambda_{i}^{\dagger}: x\right) \cdot \overline{\mathbf{E}\left(P\left|A: P_{i}\right| A_{i}: \mathbf{e}_{n}^{i}: k_{i} \cdot \Lambda+\Lambda_{i}^{\dagger}: y\right)}$. 
Here, $\sum_{m, n}^{(P)}$ means that we sum only over the $m, n$ such that the nonzero components of $\mathbf{e}_{m}^{i}, \mathbf{e}_{n}^{i}$ correspond to parabolics possessing a $\Gamma$-conjugate dominated by $P$. One may also discuss

$$
K_{\alpha}\left(P: L_{\text {dis }}^{2}\left(M / \Gamma_{M}\right): x, y: \Lambda\right)
$$

in a similar way, the explicit expression for the kernel involving no integrals and no Eisenstein series, the latter being the entry functions alone (vis-à-vis $P \in \mathscr{C}_{i}$ ).

[Note: It suffices to require that $\alpha$ be a $K$-finite element of $C_{c}^{\infty}(G)$, $K$-centrality playing no role.]

No really new ideas are needed for the proofs, the main point being a demonstration of Proposition 7.4 of TES in its daggered form, hence the attendent daggered consequence, namely the lemma appearing on p. 356 of TES. This done, no difficulty arises in writing out a daggered version of Chapter 8 of TES. The formula supra for the kernel then falls out, as do the estimates needed in the present extended setting for the analogues of Lemmas 2.1 and 2.2 as well as Proposition 2.3, the latter reading as follows.

Proposition 6.4. Let $\alpha \in C_{c}^{\infty}(G)$ be $K$-finite-then $\forall \mathbf{H} \in \mathfrak{a}_{Q}$,

$$
\operatorname{tr}\left(Q_{P}^{\mathbf{H}} \circ \operatorname{Ind}_{M \cdot A \cdot N}^{G}\left(L_{M / \Gamma_{M}}^{\text {con }} \otimes \Lambda \otimes 1\right)(\alpha) \circ Q_{P}^{\mathbf{H}}\right)
$$

is equal to

$$
\begin{aligned}
& \sum_{\mathscr{C}} \sum_{i: \mathscr{E}_{i} \in \operatorname{Dom}(P: \mathscr{C})} \sum_{\mathscr{\theta}_{i}} \frac{1}{(2 \pi)^{l^{\dagger}}} \cdot \frac{1}{*\left(\mathscr{C}_{i}^{\dagger}\right)} \\
& \times \int_{\operatorname{Re}\left(\Lambda_{i}^{\dagger}\right)=0} \sum_{m, n}^{(P)} \mathbf{C}_{m n}\left(\alpha: \mathscr{\sigma}_{i}: k_{i} \cdot \Lambda+\Lambda_{i}^{\dagger}\right) \\
& \times\left(Q_{P}^{\mathbf{H}} \mathbf{E}\left(P\left|A: P_{i}\right| A_{i}: \mathbf{e}_{m}^{i}: \Lambda_{i}^{\dagger}: ?\right), Q_{P}^{\mathbf{H}} \mathbf{E}\left(P \mid A: P_{i}: A_{i}: \mathbf{e}_{n}^{i}: \Lambda_{i}^{\dagger}: ?\right)\right)\left|d \Lambda_{i}^{\dagger}\right| .
\end{aligned}
$$

[Note: $\Lambda$ is pure imaginary.]

The above inner products

$$
\left(Q_{P}^{\mathbf{H}} \mathbf{E}(P \mid A: \ldots), Q_{P}^{\mathbf{H}} \mathbf{E}(P \mid A: \ldots)\right)
$$

are calculated as integrals over $K \times M / \Gamma_{M}$. Their explication is the next topic on our agenda.

7. Manipulation of the leading term $(P \neq G)$. The purpose of this section is to study the leading term of the inner product of two truncated partial Eisenstein series in the boldface picture, the upshot being a relative version of Proposition 5.2. In reality, the issue is primarily 
notational, the actual work having been carried out in $\S 5$. Analogous considerations will arise again in the next section when we combine what is said here with the results from the preceding section to get a boldface expression for

$$
\operatorname{tr}\left(Q^{I_{M}(\mathbf{H})} \circ L_{M / \Gamma_{M}}^{\text {con }}\left(\alpha^{P}\right) \circ Q^{I_{M}(\mathbf{H})}\right),
$$

thereby preparing the way for the cancellation. It may be helpful to keep in mind that the rationale underlying all this is the need to shift the emphasis from a given $\Gamma$-cuspidal to its containing $G$-conjugacy class. Of course, $G$ and $\{G\}$ are virtually indistinguishable, so matters simplify considerably at the top level of cuspidality!

Some of the requisite notation was introduced at the beginning of $\S 5$. To add to it, let us follow the lead in TES (p. 193) and set

$$
\left\{\begin{array}{l}
\mathbf{L}^{2}\left(\left(G \mid \mathscr{C}_{i}\right) / \Gamma\right)=\bigoplus_{\mu} L^{2}\left(G / \Gamma_{M_{i \mu}} \cdot A_{i \mu} \cdot N_{i \mu}\right), \\
\mathfrak{A}\left(\left(G \mid \mathscr{C}_{i}\right) / \Gamma\right)=\bigoplus_{\mu} \mathfrak{A}\left(G / \Gamma_{M_{i \mu}} \cdot A_{i \mu} \cdot N_{i \mu}\right) .
\end{array}\right.
$$

Elements of either of these spaces are therefore $r_{i}$-tuples. The boldface partial truncation operator then enters the picture as the map

$$
\mathbf{Q}_{\mathscr{E}_{1}}^{\mathbf{H}}: \mathfrak{A}\left(\left(G \mid \mathscr{C}_{i}\right) / \Gamma\right) \rightarrow \mathbf{L}^{2}\left(\left(G \mid \mathscr{C}_{i}\right) / \Gamma\right)
$$

defined by the rule

$$
\left(\mathbf{Q}_{\mathscr{C}_{i}}^{\mathbf{H}}\right)_{\mu}=Q_{P_{\iota \mu}}^{\mathbf{H}} .
$$

Suppose that $\mathscr{C}_{i} \succeq \mathscr{C}_{i_{0}}$. If we compose the bilinear pairing

$$
\mathbf{C}^{r_{i_{0}}} \times \mathbf{C}^{r_{i_{0}}} \rightarrow \mathbf{C}^{r_{i_{0}}}
$$

prescribed by multiplying the entries term-by-term with the reduction map

$$
\operatorname{red}\left(\mathscr{C}_{i}, \mathscr{C}_{i_{0}}\right): \mathbf{C}^{r_{i_{0}}} \rightarrow \mathbf{C}^{r_{i}}
$$

from $\S 5$, then the result is a bilinear assignment

$$
\mathbf{C}^{r_{t_{0}}} \times \mathbf{C}^{r_{t_{0}}} \rightarrow \mathbf{C}^{r_{i}}
$$

which will be denoted by the symbol $\langle ?, ?\rangle_{\mathscr{C}_{1}}$. No confusion will arise from the fact that $\mathscr{C}_{i_{0}}$ is not explicitly reflected in the notation. Intrinsic, however, to $\mathscr{C}_{i}$ is

$$
(?, ?)_{\mathscr{C}_{1}},
$$

the canonically defined $\mathbf{C}^{r_{t}}$-valued "inner product" on $\mathbf{L}^{2}\left(\left(G \mid \mathscr{C}_{i}\right) / \Gamma\right)$.

Keeping still to the supposition that $\mathscr{C}_{i} \succeq \mathscr{C}_{i_{0}}$, we can and will assume that

$$
\left(P_{i}, S_{i} ; A_{i}\right) \succeq\left(P_{i_{0}}, S_{i_{0}} ; A_{i_{0}}\right)
$$


That being, given $\boldsymbol{\Phi}_{i_{0}} \in \mathscr{E}\left(\delta, \boldsymbol{\sigma}_{i_{0}}\right)$, by

$$
\mathbf{E}\left(\mathscr{C}_{i}: P_{i_{0}} \mid A_{i_{0}}: \boldsymbol{\Phi}_{i_{0}}: \Lambda_{i_{0}}^{\dagger}: ?\right)
$$

we shall understand that element of $\mathfrak{A}\left(\left(G \mid \mathscr{C}_{i}\right) / \Gamma\right)$ whose $\mu$ th-component is the sum over those $\mu_{0}$ for which $P_{i_{0} \mu_{0}}$ has a $\Gamma$-conjugate below $P_{i \mu}$, say

$$
P_{i \mu} \succeq \gamma_{i_{0} \mu_{0}} P_{i_{0} \mu_{0}} \gamma_{i_{0} \mu_{0}}^{-1} \quad\left(\gamma_{i_{0} \mu_{0}} \in \Gamma\right)
$$

of the

$$
\begin{aligned}
& E\left(P_{i \mu}\left|A_{i \mu}: \gamma_{i_{0} \mu_{0}} P_{i_{0} \mu_{0}} \gamma_{i_{0} \mu_{0}}^{-1}\right| \gamma_{i_{0} \mu_{0}} A_{i_{0} \mu_{0}} \gamma_{i_{0} \mu_{0}}^{-1}:\right. \\
& \left.\qquad \Omega_{\gamma_{i_{0} \mu_{0}}}\left(k_{i_{0} \mu_{0}} \cdot \Lambda_{i_{0}}^{\dagger}\right) \boldsymbol{\Phi}_{i_{0} \mu_{0}}: \gamma_{i_{0} \mu_{0}} k_{i_{0} \mu_{0}} \cdot \Lambda_{i_{0}}^{\dagger}: ?\right) .
\end{aligned}
$$

Consider now two $G$-conjugacy classes $\mathscr{C}_{i_{0}^{1}}$ and $\mathscr{C}_{i_{0}^{2}}$ per $\mathscr{C}_{0}\left(\supset \mathscr{C}_{i_{0}}\right)$, both dominated by $\mathscr{C}_{i}$, so that

$$
\left(P_{i}, S_{i} ; A_{i}\right) \succeq\left\{\begin{array}{l}
\left(P_{1}, S_{1} ; A_{1}\right) \in \mathscr{C}_{i_{0}^{1}}, \\
\left(P_{2}, S_{2} ; A_{2}\right) \in \mathscr{C}_{i_{0}^{2}},
\end{array}\right.
$$

where, additionally $P_{1}^{\dagger}$ and $P_{2}^{\dagger}$ are associate, or, what amounts to the same, $W\left(A_{1}^{\dagger}, A_{2}^{\dagger}\right)$ is not empty. Fix

$$
\left\{\begin{array}{l}
\boldsymbol{\Phi}_{1} \in \mathscr{E}\left(\delta, \boldsymbol{\theta}_{i_{0}^{1}}\right) \\
\boldsymbol{\Phi}_{2} \in \mathscr{E}\left(\delta, \boldsymbol{\theta}_{i_{0}^{2}}\right)
\end{array} \quad\left(\boldsymbol{\sigma}_{i_{0}^{1}} \leftrightarrow \boldsymbol{\sigma}_{i_{0}^{2}}\right),\right.
$$

and form the partial Eisenstein series

$$
\left\{\begin{array}{l}
\mathbf{E}\left(\mathscr{C}_{i}: P_{1} \mid A_{1}: \boldsymbol{\Phi}_{1}: \Lambda_{1}^{\dagger}: ?\right) \\
\mathbf{E}\left(\mathscr{C}_{i}: P_{2} \mid A_{2}: \boldsymbol{\Phi}_{2}: \Lambda_{2}^{\dagger}: ?\right)
\end{array}\right.
$$

Then

$$
\left\{\begin{array}{l}
\mathbf{Q}_{\mathscr{C}_{1}}^{\mathbf{H}} \mathbf{E}\left(\mathscr{C}_{i}: P_{1} \mid A_{1}: \boldsymbol{\Phi}_{1}: \Lambda_{1}^{\dagger}: ?\right) \\
\mathbf{Q}_{\mathscr{C}_{i}}^{\mathrm{H}} \mathbf{E}\left(\mathscr{C}_{i}: P_{2} \mid A_{2}: \boldsymbol{\Phi}_{2}: \Lambda_{2}^{\dagger}: ?\right)
\end{array} \in L^{2}\left(\left(G \mid \mathscr{C}_{i}\right) / \Gamma\right)\right.
$$

In this situation, the inner product

$$
\left(\mathbf{Q}_{\mathscr{C}_{1}}^{\mathbf{H}} \mathbf{E}\left(\mathscr{C}_{i}: P_{1} \mid A_{1}: \boldsymbol{\Phi}_{1}: \Lambda_{1}^{\dagger}: ?\right), \mathbf{Q}_{\mathscr{C}_{1}}^{\mathbf{H}} \mathbf{E}\left(\mathscr{C}_{i}: P_{2} \mid A_{2}: \boldsymbol{\Phi}_{2}: \Lambda_{2}^{\dagger}: ?\right)\right)_{\mathscr{C}_{1}}
$$

$\left(\mathbf{H} \in \mathfrak{a}_{Q}\right)$

is not a scalar but an $r_{i}$-tuple (cf. supra). If $\Lambda_{1}^{\dagger}$ and $\Lambda_{2}^{\dagger}$ are pure imaginary and in general position, then it follows from $\S 8$ of [2-(d)] that its "leading term" is the sum

$$
(-1)^{l_{0}^{\dagger}} \cdot \sum_{i_{0}: P_{t_{0}} \preceq P_{i}} \operatorname{vol}\left(\mathscr{C}_{i_{0}}^{\dagger}\right) \cdot \sum_{w_{t_{0}: 1}^{\dagger} \in W\left(A_{t_{0}}^{\dagger}, A_{1}^{\dagger}\right)} \sum_{w_{i_{0}: 2}^{\dagger} \in W\left(A_{i_{0}}^{\dagger}, A_{2}^{\dagger}\right)}
$$


of the

$$
\left(1 / \prod_{\lambda_{i_{0}}^{\dagger}}\left(w_{i_{0}: 1}^{\dagger} \Lambda_{1}^{\dagger}+w_{i_{0}: 2}^{\dagger} \bar{\Lambda}_{2}^{\dagger}, \lambda_{i_{0}}^{\dagger}\right)\right)
$$

times

$$
\begin{aligned}
& \left\langle\mathbf{e}\left(\mathscr{C}_{i_{0}}: \mathbf{H}: w_{i_{0}: 1}^{\dagger} \Lambda_{1}^{\dagger}+w_{i_{0}: 2}^{\dagger} \bar{\Lambda}_{2}^{\dagger}\right),\right. \\
& \left(\mathbf{c}\left(P_{i_{0}}\left|A_{i_{0}}: P_{1}\right| A_{1}: w_{i_{0}: 1}^{\dagger}: \Lambda_{1}^{\dagger}\right) \boldsymbol{\Phi}_{1},\right. \\
& \left.\left.\quad \mathbf{c}\left(P_{i_{0}}\left|A_{i_{0}}: P_{2}\right| A_{2}: w_{i_{0}: 2}^{\dagger}: \Lambda_{2}^{\dagger}\right) \boldsymbol{\Phi}_{2}\right)_{\mathscr{E}_{i_{0}}}\right\rangle_{\mathscr{E}_{1}}
\end{aligned}
$$

The analogue of Proposition 5.2 in the relative setting is also essentially immediate, viz.:

Proposition 7.1. Fix

$$
\left\{\begin{array}{l}
\Lambda_{1}^{\dagger} \in \sqrt{-1} \check{\mathfrak{a}}_{1}^{\dagger}, \\
\Lambda_{2}^{\dagger} \in \sqrt{-1} \check{\mathfrak{a}}_{2}^{\dagger} .
\end{array}\right.
$$

Then $\bmod \mathrm{o}(\mathbf{H})$

$$
\left(\mathbf{Q}_{\mathscr{C}_{i}}^{\mathbf{H}} \mathbf{E}\left(\mathscr{C}_{i}: P_{1} \mid A_{1}: \boldsymbol{\Phi}_{1}: \Lambda_{1}^{\dagger}: ?\right), \mathbf{Q}_{\mathscr{C}_{i}}^{\mathbf{H}} \mathbf{E}\left(\mathscr{C}_{i}: P_{2} \mid A_{2}: \boldsymbol{\Phi}_{2}: \Lambda_{2}^{\dagger}: ?\right)\right)_{\mathscr{C}_{l}}
$$

$\left(\mathbf{H} \in \mathfrak{a}_{Q}\right)$

equals $(-1)_{0}^{l_{0}^{\dagger}}$ times the sum over

$$
w_{12}^{\dagger} \in W\left(A_{1}^{\dagger}, A_{2}^{\dagger}\right)
$$

of the sum

$$
\sum_{\left(i_{0}, w_{i_{0}}^{0}\right)} \sum_{\left(j_{0}, w_{j_{0} t_{0}}^{\dagger \prime}\right)}
$$

of the

$$
\begin{aligned}
& (-1)^{\#\left(\Sigma_{P_{0}^{\dagger^{\prime}}}^{0}\right)} \cdot D_{*}^{\Sigma_{P_{J_{0}}^{\dagger^{\prime}}}^{0}}\left[\frac{\operatorname{vol}\left(\mathscr{C}_{i^{\prime}}^{\dagger}\right)}{\prod_{\lambda_{j_{0}}^{\dagger} \in \Sigma_{P_{J_{0}}^{\dagger}}^{0}-\Sigma_{P_{J_{0}}^{\dagger^{\prime}}}^{0}\left(?, \lambda_{j_{0}}^{\dagger}\right)}}\right. \\
& \left.\times\left\langle\mathbf{e}\left(\mathscr{C}_{j_{0}}: \mathbf{H}: ?\right),\left(1_{j_{0} i_{0}}(?), 2_{j_{0} i_{0}}(?)\right)_{\mathscr{C}_{0}}\right\rangle \mathscr{\mathscr { C }}_{i}\right]\left.\right|_{w_{j_{0} i_{0}}^{\dagger^{\prime}} w_{l_{0}}^{0}\left(\Lambda_{1}^{\dagger}+w_{12}^{\dagger} \bar{\Lambda}_{2}^{\dagger}\right)} .
\end{aligned}
$$

REMARKS. (1) The superscript "zero" has not been appended to $\Lambda_{1}^{\dagger}$ and $\Lambda_{2}^{\dagger}$, although it has been otherwise retained. 
(2) There are two daggering operations taking place: $\dagger$ and $\dagger$. The new one, $\nmid$, is vis-à-vis a generic domination

$$
P_{j_{0}} \preceq P^{\prime} \quad\left(\preceq P_{i}\right) .
$$

(3) $P^{\prime}$ enters in qua the notion of "daggered association class" (cf. $\S 5$ ). Because daggering into $M^{\prime}$ is the same as daggering into $M_{i}$ and then into ' $M=M^{\prime}$, the daggered association class is a function solely of $P^{\prime}$.

(4) $1_{j_{0} i_{0}}$ is again a constant, namely

$$
\mathbf{c}\left(P_{j_{0}}\left|A_{j_{0}}: P_{1}\right| A_{1}: w_{j_{0} i_{0}}^{\dagger \prime} w_{i_{0}}^{0}: \Lambda_{1}^{\dagger}\right) \boldsymbol{\Phi}_{1}
$$

while $2_{j_{0} i_{0}}$ is, as before,

$$
\begin{gathered}
\mathbf{c}\left(P_{i_{0}}\left|A_{i_{0}}: P_{j_{0}}\right| A_{j_{0}}: w_{j_{0} i_{0}}^{-\dagger \prime}: w_{j_{0} i_{0}}^{\dagger \dagger} w_{i_{0}}^{0} \Lambda_{1}^{\dagger}-\Lambda_{j_{0}}^{\dagger}\right)^{*} \\
\cdot \mathbf{c}\left(P_{i_{0}} \mid A_{i_{0}}: P_{2}: A_{2}: w_{i_{0}}^{0} w_{12}^{\dagger}: \Lambda_{2}^{\dagger}\right) \mathbf{\Phi}_{2} .
\end{gathered}
$$

(5) The c-functions are "block diagonal" in the sense that the $i_{0} \mu_{0}$ to $j_{0} \nu_{0}$ entry is zero unless $\mu_{0}$ and $\nu_{0}$ give the same $\mu$ per $\mathscr{C}_{i}$.

(6) While the above result is a consequence of Proposition 5.2 (applied to the $M_{i \mu}$ ) and a diagram chase, it is an important point that the c-functions are precisely those of Proposition 5.2.

8. Consolidation. The purpose of this section is to take the mod $\mathbf{o}(\mathbf{H})$ expression for

$$
\operatorname{tr}\left(Q^{\mathbf{H}} \circ L_{G / \Gamma}^{\text {dis }}(\alpha) \circ Q^{\mathbf{H}}\right),
$$

as formulated in $\S 8$ of [2-(f)], and recast it into the boldface mold. This shift in emphasis will be exploited in the next section when we take up the integral manipulations.

Let $\alpha$ be a $K$-central, $K$-finite element of $C_{c}^{\infty}(G)$-then the central conclusion of $[2-(\mathbf{f})]$ is that $\bmod o(\mathbf{H})$

$$
\operatorname{tr}\left(Q^{\mathbf{H}} \circ L_{G / \Gamma}^{\text {dis }}(\alpha) \circ Q^{\mathbf{H}}\right)
$$

is the same as

$$
\mathbf{K}\left(\mathbf{H}_{\mathrm{O}}: \alpha: \Gamma\right)
$$

less

$$
\begin{array}{r}
\sum_{i=1}^{r-1}(-1)^{\operatorname{rank}\left(P_{\imath}\right)} p\left(\Gamma: P_{i}: \mathbf{H}\left(P_{i}\right)-\mathbf{H}_{\mathrm{O}}\left(P_{i}\right)\right) \\
\cdot \operatorname{tr}\left(Q^{I_{M_{i}}(\mathbf{H})} \circ L_{M_{\imath} / \Gamma_{M_{l}}}^{\mathrm{dis}}\left(\alpha^{P_{\imath}}\right) \circ Q^{I_{M_{i}}(\mathbf{H})}\right)
\end{array}
$$


less

$$
\begin{gathered}
\sum_{i=1}^{r}(-1)^{\operatorname{rank}\left(P_{l}\right)} p\left(\Gamma: P_{i}: \mathbf{H}\left(P_{i}\right)-\mathbf{H}_{\mathrm{O}}\left(P_{i}\right)\right) \\
\cdot \operatorname{tr}\left(Q^{I_{M_{i}}(\mathbf{H})} \circ L_{M_{\imath} / \Gamma_{M_{l}}}^{\text {con }}\left(\alpha^{P_{l}}\right) \circ Q^{I_{M_{l}}(\mathbf{H})}\right) .
\end{gathered}
$$

Here $\sum_{i=1}^{r}$ is a sum over a set of representatives for the $\Gamma$-conjugacy classes of $\Gamma$-cuspidal split parabolic subgroups of $G, r$ the index associated with $G$ itself. The exact definition of

$$
\mathbf{K}\left(\mathbf{H}_{\mathrm{O}}: \alpha: \Gamma\right)
$$

is of no importance for the considerations at hand, hence need not be detailed. As for the other terms, we recall only that $p\left(\Gamma: P_{i}: ?\right)$ is an Arthur polynomial (cf. $\S 7$ of [2-(f)]).

To facilitate our transition, some more notation will be needed.

Given $\mathscr{C}_{i}$, let

$$
\mathbf{L}^{2}\left(\mathscr{C}_{i} / \Gamma_{\mathscr{C}_{i}}\right)=\bigoplus_{\mu} L^{2}\left(M_{i \mu} / \Gamma_{M_{i \mu}}\right),
$$

the obvious interpretations then being assigned to the symbols

$$
\left\{\begin{array} { l } 
{ \mathbf { L } _ { \text { dis } } ^ { 2 } ( \mathscr { C } _ { i } / \Gamma _ { \mathscr { C } _ { i } } ) , } \\
{ \mathbf { L } _ { \text { con } } ^ { 2 } ( \mathscr { C } _ { i } / \Gamma _ { \mathscr { C } _ { i } } ) , }
\end{array} \quad \left\{\begin{array}{l}
\mathbf{L}_{\mathscr{E}_{i} / \Gamma_{\mathscr{I}_{i}}}^{\text {dis }}, \\
\mathbf{L}_{\mathscr{C}_{i} / \Gamma_{\mathscr{C}_{i}}}^{\text {con }} .
\end{array}\right.\right.
$$

Relative to a $K$-central, $K$-finite element $\alpha$ of $C_{c}^{\infty}(G)$, put

$$
\mathbf{L}_{\mathscr{C}_{\imath} / \Gamma_{\mathscr{E}_{i}}}\left(\alpha^{\mathscr{C}_{\imath}}\right)=\bigoplus_{\mu} L_{M_{\imath \mu} / \Gamma_{M_{\imath \mu}}}\left(\alpha^{P_{\imath \mu}}\right)
$$

so that, with the evident convention,

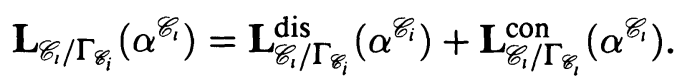

We shall agree to write

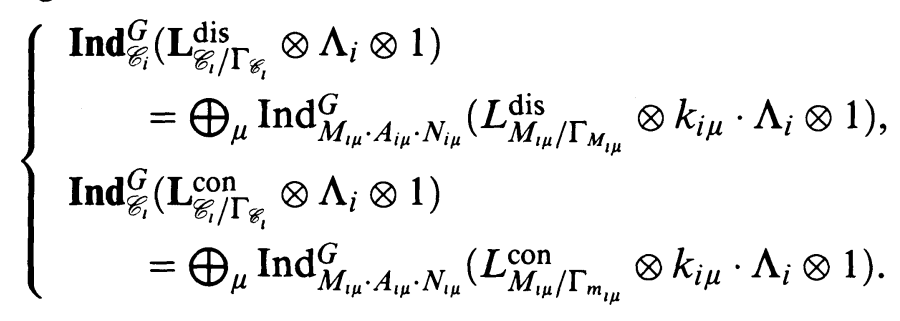

The $\mathscr{C}_{i}$-analogue of $Q_{P}^{\mathbf{H}}$ was introduced in $\S 7: \mathbf{Q}_{\mathscr{C}_{i}}^{\mathbf{H}}$. It will also be necessary to employ the $\mathscr{C}_{i}$-analogue of $Q^{I_{M}(\mathbf{H})}$, namely: $\mathbf{Q}^{I_{\mathscr{I}_{l}}(\mathbf{H})}$, the definition of which is clear. Finally, let

a boldface Arthur polynomial.

$$
\mathbf{p}\left(\Gamma: \mathscr{C}_{i}: \mathbf{H}\right)=\left(p\left(\Gamma: P_{i \mu}: \mathbf{H}\left(P_{i \mu}\right)\right)\right)
$$


If the $\mathscr{H}_{\mu}$ are Hilbert spaces and if the $T_{\mu \nu}: \mathscr{H}_{\mu} \rightarrow \mathscr{H}_{\nu}$ are trace class operators, then with

$$
\left\{\begin{array}{l}
\mathscr{X}=\bigoplus_{\mu} \mathscr{H}_{\mu}, \\
\mathbf{T}=\bigoplus_{\mu \nu} T_{\mu \nu}, \quad \mathbf{T}: \mathscr{X} \rightarrow \mathscr{X},
\end{array}\right.
$$

we define

$$
\operatorname{tr}_{\mathscr{C}_{i}}(\mathbf{T}) \in \mathbf{C}^{r_{i}}
$$

as the $r_{i}$-tuple whose $\mu$ th-entry is $\operatorname{tr}\left(T_{\mu \mu}\right)$.

Having established the notation, it can now be said that $\bmod \mathbf{o}(\mathbf{H})$

$$
\operatorname{tr}\left(Q^{\mathbf{H}} \circ L_{G / \Gamma}^{\mathrm{dis}}(\alpha) \circ Q^{\mathbf{H}}\right)
$$

is the same as

$$
\mathbf{K}\left(\mathbf{H}_{\mathrm{O}}: \alpha: \Gamma\right)
$$

less

$$
\begin{aligned}
& \sum_{\mathscr{E}_{l}}(-1)^{\operatorname{rank}\left(\mathscr{E}_{1}\right)} \\
& \cdot\left\langle\mathbf{p}\left(\Gamma: \mathscr{C}_{i}: \mathbf{H}-\mathbf{H}_{\mathrm{O}}\right), \mathbf{t r}_{\mathscr{C}_{l}}\left(\mathbf{Q}^{I_{\mathscr{E}_{i}}(\mathbf{H})} \circ \mathbf{L}_{\mathscr{C}_{i} / \Gamma_{\mathscr{C}_{l}}}^{\text {dis }}\left(\alpha^{\mathscr{C}_{i}}\right) \circ \mathbf{Q}^{I_{\mathscr{E}_{l}}(\mathbf{H})}\right)\right\rangle
\end{aligned}
$$

less

$$
\begin{aligned}
& \sum_{\mathscr{C}_{i}}(-1)^{\operatorname{rank}\left(\mathscr{C}_{l}\right)} \\
& \quad \cdot\left\langle\mathbf{p}\left(\Gamma: \mathscr{C}_{i}: \mathbf{H}-\mathbf{H}_{\mathrm{O}}\right), \operatorname{tr}_{\mathscr{C}_{i}}\left(\mathbf{Q}^{I_{\mathscr{E}_{i}}(\mathbf{H})} \circ \mathbf{L}_{\mathscr{C}_{i} / \Gamma_{\mathscr{C}_{i}}}^{\operatorname{con}}\left(\alpha^{\mathscr{C}_{i}}\right) \circ \mathbf{Q}^{I_{\mathscr{E}_{i}}(\mathbf{H})}\right)\right\rangle .
\end{aligned}
$$

ReMARKs. (1) Both sums are over $G$-conjugacy classes (not association classes), the slash through the summation sign meaning that the sum is over the $\mathscr{C}_{i} \neq\{G\}$.

(2) The angle brackets refer to the ordinary pairing

$$
\mathbf{C}^{r_{i}} \times \mathbf{C}^{r_{i}} \rightarrow \mathbf{C} \text {. }
$$

Holding

$$
\operatorname{tr}_{\mathscr{C}_{i}}\left(\mathbf{Q}^{I_{\mathscr{E}_{1}}(\mathbf{H})} \circ \mathbf{L}_{\mathscr{C}_{i} / \Gamma_{\mathscr{C}_{i}}}^{\text {dis }}\left(\alpha^{\mathscr{C}_{i}}\right) \circ \mathbf{Q}^{I_{\mathscr{E}_{i}}(\mathbf{H})}\right)
$$

in abeyance, let us consider

$$
\operatorname{tr}_{\mathscr{E}_{1}}\left(\mathbf{Q}^{I_{\mathscr{E}_{1}}(\mathbf{H})} \circ \mathbf{L}_{\mathscr{C}_{l} / \Gamma_{\mathscr{I}_{l}}}^{\text {con }}\left(\alpha^{\mathscr{C}_{l}}\right) \circ \mathbf{Q}^{I_{\mathscr{E}_{l}}(\mathbf{H})}\right)
$$

in more detail. It is to be noted that the discussion infra admits the special case $\mathscr{C}_{i}=\{G\}$.

Interpreting Theorem 6.2 in the present context, we can start by saying that

$$
\operatorname{tr}_{\mathscr{C}_{1}}\left(\mathbf{Q}^{I_{\mathscr{E}_{1}}(\mathbf{H})} \circ \mathbf{L}_{\mathscr{C}_{l} / \Gamma_{\mathscr{E}_{1}}}^{\text {con }}\left(\alpha^{\mathscr{C}_{1}}\right) \circ \mathbf{Q}^{I_{\mathscr{E}_{l}}(\mathbf{H})}\right)
$$


equals

$$
\frac{1}{(2 \pi)^{l_{i}}} \cdot \int_{\operatorname{Re}\left(\Lambda_{i}\right)=0} \operatorname{tr}_{\mathscr{C}_{i}}\left(\mathbf{Q}_{\mathscr{C}_{i}}^{\mathbf{H}} \circ \operatorname{Ind}_{\mathscr{C}_{i}}^{G}\left(\mathbf{L}_{\mathscr{C}_{i} / \Gamma_{\mathscr{E}_{i}}}^{\text {con }} \otimes \Lambda_{i} \otimes 1\right)(\alpha) \circ \mathbf{Q}_{\mathscr{C}_{i}}^{\mathbf{H}}\right)\left|d \Lambda_{i}\right| .
$$

Thus, we are reduced to looking at

$$
\operatorname{tr}_{\mathscr{C}_{l}}\left(\mathbf{Q}_{\mathscr{E}_{i}}^{\mathbf{H}} \circ \mathbf{I n d}_{\mathscr{G}_{i}}^{G}\left(\mathbf{L}_{\mathscr{E}_{l} / \Gamma_{\mathscr{I}_{l}}}^{\text {con }} \otimes \Lambda_{i} \otimes 1\right)(\alpha) \circ \mathbf{Q}_{\mathscr{C}_{i}}^{\mathbf{H}}\right),
$$

the study of which falls naturally into two parts.

Put

$$
\mathbf{C}_{m n}\left(\alpha: \boldsymbol{\theta}_{i_{0}}, \Lambda_{i_{0}}\right)_{\mathscr{C}_{\imath}}=\operatorname{red}\left(\mathscr{C}_{i}, \mathscr{C}_{i_{0}}\right) \mathbf{C}_{m n}\left(\alpha: \boldsymbol{\theta}_{i_{0}}, \Lambda_{i_{0}}\right) \mathscr{\mathscr { C }}_{i_{0}}
$$

where, in turn,

$$
\mathbf{C}_{m n}\left(\alpha: \boldsymbol{\theta}_{i_{0}}, \Lambda_{i_{0}}\right)_{\mathscr{i}_{i_{0}}}
$$

is that element of $\mathbf{C}^{r_{0}}$ defined as follows:

(a) If $\mathbf{e}_{m}^{i_{0}}, \mathbf{e}_{n}^{i_{0}}$ do not correspond to the same $P_{i_{0} \mu_{0}}$, then

$$
\mathbf{C}_{m n}\left(\alpha: \boldsymbol{\sigma}_{i_{0}}, \Lambda_{i_{0}}\right)_{\mathscr{C}_{i_{0}}}=0
$$

(b) If $\mathbf{e}_{m}^{i_{0}}, \mathbf{e}_{n}^{i_{0}}$ do correspond to the same $P_{i_{0} \mu_{0}}$, then the $\mu_{0}$ th-entry of

$$
\mathbf{C}_{m n}\left(\alpha: \boldsymbol{\theta}_{i_{0}}, \Lambda_{i_{0}}\right)_{\mathscr{C}_{i_{0}}}
$$

is

$$
\mathbf{C}_{m n}\left(\alpha: \boldsymbol{\theta}_{i_{0}} \cdot \Lambda_{i_{0}}\right)
$$

all the other entries being zero.

Owing now to Proposition 6.4,

$$
\operatorname{tr}_{\mathscr{E}_{l}}\left(\mathbf{Q}_{\mathscr{C}_{i}}^{\mathbf{H}} \circ \mathbf{I n d}_{\mathscr{C}_{i}}^{G}\left(\mathbf{L}_{\mathscr{C}_{i} / \Gamma_{\mathscr{C}_{i}}}^{\text {con }} \otimes \Lambda_{i} \otimes 1\right)(\alpha) \circ \mathbf{Q}_{\mathscr{C}_{i}}^{\mathbf{H}}\right)
$$

can be rewritten as

$$
\begin{aligned}
& \sum_{\mathscr{E}_{I_{0}}: \mathscr{E}_{i_{0}} \prec \mathscr{C}_{i}} \sum_{\mathscr{\sigma}_{i_{0}}} \frac{1}{(2 \pi)^{l_{0}^{\dagger}}} \cdot \frac{1}{*\left(\mathscr{C}_{i_{0}}^{\dagger}\right)} \\
& \times \int_{\operatorname{Re}\left(\Lambda_{i_{0}}^{\dagger}\right)=0}\left\langle\sum_{m, n} \mathbf{C}_{m n}\left(\alpha: \boldsymbol{\theta}_{i_{0}}, \Lambda_{i}+\Lambda_{i_{0}}^{\dagger}\right) \mathscr{\mathscr { C }}_{i},\right. \\
& \times\left(\mathbf{Q}_{\mathscr{C}_{i}}^{\mathbf{H}} \mathbf{E}\left(\mathscr{C}_{i}: P_{i_{0}} \mid A_{i_{0}}: \mathbf{e}_{m}^{i_{0}}: \Lambda_{i_{0}}^{\dagger}: ?\right),\right. \\
& \left.\left.\mathbf{Q}_{\mathscr{C}_{1}}^{\mathbf{H}} \mathbf{E}\left(\mathscr{C}_{i}: P_{i_{0}} \mid A_{i_{0}}: \mathbf{e}_{n}^{i_{0}}: \Lambda_{i_{0}}^{\dagger}: ?\right)\right)_{\mathscr{C}_{l}}\right\rangle_{\mathscr{C}_{l}}\left|d \Lambda_{i_{0}}^{\dagger}\right| \text {. }
\end{aligned}
$$

[Note: The angle bracket $\langle\text { ?, ? }\rangle_{\mathscr{C}_{\ell}}$ has the same significance as in $\S 7$ but, of course, with $\mathscr{C}_{i}=\mathscr{C}_{i_{0}}$.] 
To complete the explication of

$$
\operatorname{tr}_{\mathscr{C}_{i}}\left(\mathbf{Q}_{\mathscr{C}_{i}}^{\mathbf{H}} \circ \mathbf{I n d}_{\mathscr{C}_{i}}^{G}\left(\mathbf{L}_{\mathscr{C}_{l} / \Gamma_{\mathscr{E}_{i}}}^{\text {con }} \otimes \Lambda_{i} \otimes 1\right)(\alpha) \circ \mathbf{Q}_{\mathscr{C}_{i}}^{\mathbf{H}}\right)
$$

one need only deal with

$$
\left(\mathbf{Q}_{\mathscr{C}_{l}}^{\mathbf{H}} \mathbf{E}\left(\mathscr{C}_{i}: P_{i_{0}} \mid A_{i_{0}}: \mathbf{e}_{m}^{i_{0}}: \Lambda_{i_{0}}^{\dagger}: ?\right), \mathbf{Q}_{\mathscr{C}_{1}}^{\mathbf{H}} \mathbf{E}\left(\mathscr{C}_{i}: P_{i_{0}} \mid A_{i_{0}}: \mathbf{e}_{n}^{i_{0}}: \Lambda_{i_{0}}^{\dagger}: ?\right)\right)_{\mathscr{C}_{i}} \cdot
$$

This, however, is simply a matter of quoting Proposition 7.1, at least in so far as the leading term is concerned.

9. An integral formula. The purpose of this section is to discuss the contribution to

$$
\operatorname{tr}\left(Q^{\mathbf{H}} \circ L_{G / \Gamma}^{\text {dis }}(\alpha) \circ Q^{\mathbf{H}}\right)
$$

associated with

$$
\begin{aligned}
& \sum_{\mathscr{C}_{I}}(-1)^{\operatorname{rank}\left(\mathscr{C}_{i}\right)} \\
& \quad \cdot\left\langle\mathbf{p}\left(\Gamma: \mathscr{C}_{i}: \mathbf{H}-\mathbf{H}_{\mathrm{O}}\right), \mathbf{t r}_{\mathscr{C}_{i}}\left(\mathbf{Q}^{I_{\mathscr{E}_{i}}(\mathbf{H})} \circ \mathbf{L}_{\mathscr{C}_{i} / \Gamma_{\mathscr{C}_{i}}}^{\text {dis }}\left(\alpha^{\mathscr{C}_{I}}\right) \circ \mathbf{Q}^{I_{\mathscr{I}_{i}}(\mathbf{H})}\right)\right\rangle .
\end{aligned}
$$

and

$$
\begin{aligned}
& \sum_{\mathscr{C}_{1}}(-1)^{\operatorname{rank}\left(\mathscr{C}_{I}\right)} \\
& \quad \cdot\left\langle\mathbf{p}\left(\Gamma: \mathscr{C}_{i}: \mathbf{H}-\mathbf{H}_{\mathrm{O}}\right), \operatorname{tr}_{\mathscr{C}_{l}}\left(\mathbf{Q}^{I_{\mathscr{I}_{l}}(\mathbf{H})} \circ \mathbf{L}_{\mathscr{C}_{i} / \Gamma_{\mathscr{C}_{i}}}^{\operatorname{con}}\left(\alpha^{\mathscr{C}_{l}}\right) \circ \mathbf{Q}^{I_{\mathscr{I}_{l}}(\mathbf{H})}\right)\right\rangle .
\end{aligned}
$$

Here, as always, $\alpha$ is a $K$-central, $K$-finite element of $C_{c}^{\infty}(G)$. We want now to let $\mathbf{H} \rightarrow-\infty$. Ignoring anything that is $\mathbf{o}(\mathbf{H})$, the essential idea of the cancellation is to eliminate all terms which blow up as $\mathbf{H} \rightarrow-\infty$. The terms that remain may or may not depend on $\mathbf{H}_{\mathrm{O}}$. Those that do will eventually be connected with their analogues from

$$
\mathbf{K}\left(\mathbf{H}_{\mathrm{O}}: \alpha: \Gamma\right)
$$

while those that do not are in their final form. Our present objective will be to make the integral manipulations that constitute an essential preliminary for drawing this conclusion, culminating in Theorem 9.7.

For the time being, let us fix a $\mathscr{C}_{i}$ and consider

$$
\left\langle\mathbf{p}\left(\Gamma: \mathscr{C}_{i}: \mathbf{H}-\mathbf{H}_{\mathrm{O}}\right), \mathbf{t r}_{\mathscr{C}_{i}}\left(\mathbf{Q}^{I_{\mathscr{I}_{i}}(\mathbf{H})} \circ \mathbf{L}_{\mathscr{C}_{1} / \Gamma_{\mathscr{E}_{1}}}^{\text {con }}\left(\alpha^{\mathscr{C}_{i}}\right) \circ \mathbf{Q}^{I_{\mathscr{E}_{1}}(\mathbf{H})}\right)\right\rangle
$$

On the basis of what has been said in $\S 8$, we can write

$$
\operatorname{tr}_{\mathscr{C}_{i}}\left(\mathbf{Q}^{I_{\mathscr{E}_{i}}(\mathbf{H})} \circ \mathbf{L}_{\mathscr{C}_{i} / \Gamma_{\mathscr{E}_{1}}}^{\text {con }}\left(\alpha^{\mathscr{C}_{i}}\right) \circ \mathbf{Q}^{I_{\mathscr{E}_{i}}(\mathbf{H})}\right)
$$

as

$$
\frac{1}{(2 \pi)^{l_{i}}} \cdot \int_{\operatorname{Re}\left(\Lambda_{i}\right)=0} \operatorname{tr}_{\mathscr{C}_{i}}\left(\mathbf{Q}_{\mathscr{E}_{i}}^{\mathbf{H}} \circ \mathbf{I n d}_{\mathscr{C}_{i}}^{G}\left(\mathbf{L}_{\mathscr{C}_{i} / \Gamma_{\mathscr{E}_{i}}}^{\text {con }} \otimes \Lambda_{i} \otimes 1\right)(\alpha) \circ \mathbf{Q}_{\mathscr{C}_{i}}^{\mathbf{H}}\right)\left|d \Lambda_{i}\right|,
$$


where

$$
\operatorname{tr}_{\mathscr{C}_{1}}\left(\mathbf{Q}_{\mathscr{E}_{i}}^{\mathbf{H}} \circ \mathbf{I n d}_{\mathscr{C}_{1}}^{G}\left(\mathbf{L}_{\mathscr{C}_{i} / \Gamma_{\mathscr{C}_{1}}}^{\text {con }} \otimes \Lambda_{i} \otimes 1\right)(\alpha) \circ \mathbf{Q}_{\mathscr{C}_{1}}^{\mathbf{H}}\right)
$$

is equal to

$$
\begin{aligned}
& \sum_{\mathscr{E}_{\imath_{0}}: \mathscr{E}_{\mathfrak{I}_{0}} \prec \mathscr{C}_{1}} \sum_{\boldsymbol{\theta}_{\iota_{0}}} \frac{1}{(2 \pi)^{l_{0}^{\dagger}}} \cdot \frac{1}{*\left(\mathscr{C}_{i_{0}}^{\dagger}\right)} \\
& \times \int_{\operatorname{Re}\left(\Lambda_{i_{0}}^{\dagger}\right)=0}\left\langle\sum_{m, n} \mathbf{C}_{m n}\left(\alpha: \boldsymbol{\theta}_{i_{0}}, \Lambda_{i}+\Lambda_{i_{0}}^{\dagger}\right) \mathscr{\mathscr { C }}_{i},\right. \\
& \left(\mathbf{Q}_{\mathscr{C}_{1}}^{\mathrm{H}} \mathbf{E}\left(\mathscr{C}_{i}: P_{i_{0}} \mid A_{i_{0}}: \mathbf{e}_{m}^{i_{0}}: \Lambda_{i_{0}}^{\dagger}: ?\right),\right. \\
& \left.\left.\mathbf{Q}_{\mathscr{C}_{l}}^{\mathbf{H}} \mathbf{E}\left(\mathscr{C}_{i}: P_{i_{0}} \mid A_{i_{0}}: \mathbf{e}_{n}^{i_{0}}: \Lambda_{i_{0}}^{\dagger}: ?\right)\right)_{\mathscr{C}_{1}}\right\rangle_{\mathscr{C}_{l}}\left|d \Lambda_{i_{0}}^{\dagger}\right| \text {, }
\end{aligned}
$$

and in turn, thanks to Proposition 7.1, $\bmod \mathbf{o}_{\mathscr{E}_{i}}\left(\boldsymbol{\sigma}_{i_{0}}, \Lambda_{i}+\Lambda_{i_{0}}^{\dagger}: \mathbf{H}\right)$

$$
\begin{aligned}
& \left\langle\sum_{m, n} C_{m n}\left(\alpha: \boldsymbol{\theta}_{i_{0}}, \Lambda_{i}+\Lambda_{i_{0}}^{\dagger}\right) \mathscr{\mathscr { C }}_{\imath},\right. \\
& \left(\mathbf{Q}_{\mathscr{C}_{l}}^{\mathrm{H}} \mathbf{E}\left(\mathscr{C}_{i}: P_{i_{0}} \mid A_{i_{0}}: \mathbf{e}_{m}^{i_{0}}: \Lambda_{i_{0}}^{\dagger}: ?\right),\right. \\
& \left.\left.\mathbf{Q}_{\mathscr{C}_{i}}^{\mathbf{H}} \mathbf{E}\left(\mathscr{C}_{i}: P_{i_{0}} \mid A_{i_{0}}: \mathbf{e}_{n}^{i_{0}}: \Lambda_{i_{0}}^{\dagger}: ?\right)\right)_{\mathscr{C}_{i}}\right\rangle_{\mathscr{C}_{l}}
\end{aligned}
$$

is equal to $(-1)^{l_{0}^{\dagger}}$ times the sum

$$
\sum_{m, n} \sum_{w_{\imath_{0}}^{\dagger}} \sum_{\left(j_{0}, w_{\jmath_{0}}^{0}\right)} \sum_{\left(k_{0}, w_{k_{0} j_{0}}^{\dagger \prime}\right)}
$$

of the

$$
\begin{aligned}
& (-1)^{\#\left(\Sigma_{k_{0}}^{+}\right)} \cdot D_{*}^{\Sigma_{P_{k_{0}}^{+\prime}}^{0}}\left[\frac{\operatorname{vol}\left(\mathscr{C}_{i^{\prime}}^{\dagger}\right)}{\prod_{\lambda_{k_{0}}^{\dagger} \in \Sigma_{P_{k_{0}}^{\dagger}}^{0}-\Sigma_{P_{k_{0}}^{\dagger}}^{0}\left(?, \lambda_{k_{0}}^{\dagger}\right)}}\right. \\
& \times\left\langle\mathbf{C}_{m n}\left(\alpha: \boldsymbol{\theta}_{i_{0}}, \Lambda_{i}+\Lambda_{i_{0}}^{\dagger}\right) \mathscr{\mathscr { C }}_{\mathscr{I}},\right. \\
& \left.\left.\left\langle\mathbf{e}\left(\mathscr{C}_{k_{0}}: \mathbf{H}: ?\right),\left(1_{k_{0} j_{0}}(?), 2_{k_{0} j_{0}}(?)\right)_{\mathscr{C}_{k_{0}}}\right\rangle_{\mathscr{C}_{i}}\right\rangle \mathscr{\mathscr { C }}_{l}\right]\left.\right|_{w_{k_{0} J_{0}}^{\dagger \prime} w_{J_{0}}^{0}\left(1-w_{\iota_{0}}^{\dagger}\right) \Lambda_{\iota_{0}}^{\dagger}}
\end{aligned}
$$

Remarks. (1) Here, $P_{1}$ and $P_{2}$ are the same, viz. $P_{i_{0}}$, while $\Lambda_{1}^{\dagger}$ and $\Lambda_{2}^{\dagger}$ are the same, viz. $\Lambda_{i_{0}}^{\dagger}$. Thus, $w_{i_{0}}^{\dagger}$ runs through $W\left(A_{i_{0}}^{\dagger}\right)$, the 
evaluation then taking place at

$$
w_{k_{0} j_{0}}^{\dagger \prime} w_{j_{0}}^{0}\left(1-w_{i_{0}}^{\dagger}\right) \Lambda_{i_{0}}^{\dagger} \text {. }
$$

(2) In the current setting, $1_{k_{0} j_{0}}$ is

$$
\mathbf{c}\left(P_{k_{0}}\left|A_{k_{0}}: P_{i_{0}}\right| A_{i_{0}}: w_{k_{0} j_{0}}^{\dagger \prime} w_{j_{0}}^{0}: \Lambda_{i_{0}}^{\dagger}\right) \mathbf{e}_{m}^{i_{0}}
$$

and $2_{k_{0} j_{0}}$ evaluated at $\Lambda_{k_{0}}^{\dagger}$ is

$$
\begin{gathered}
\mathbf{c}\left(P_{j_{0}}\left|A_{j_{0}}: P_{k_{0}}\right| A_{k_{0}}: w_{k_{0} j_{0}}^{-\dagger \prime}: w_{k_{0} j_{0}}^{\dagger} w_{j_{0}}^{0} \Lambda_{i_{0}}^{\dagger}-\Lambda_{k_{0}}^{\dagger}\right)^{*} \\
\cdot \mathbf{c}\left(P_{j_{0}}\left|A_{j_{0}}: P_{i_{0}}\right| A_{i_{0}}: w_{j_{0}}^{0} w_{i_{0}}^{\dagger}: \Lambda_{i_{0}}^{\dagger}\right) \mathbf{e}_{n}^{i_{0}} .
\end{gathered}
$$

The integrand in

$$
\int_{\operatorname{Re}\left(\Lambda_{0}^{\dagger}\right)=0} \cdots
$$

can be simplified considerably. Employing a suggestive notation (cf. $\S 8)$, write

$$
\begin{aligned}
\mathbf{C}_{m n} & \left(\alpha: \boldsymbol{\theta}_{i_{0}}, \Lambda_{i}+\Lambda_{i_{0}}^{\dagger}\right) \mathscr{\mathscr { C }}_{l} \\
& =\left(\operatorname{Ind}_{\mathscr{C}_{i_{0}}}^{G}\left(\left(\boldsymbol{\sigma}_{i_{0}}, \Lambda_{i}+\Lambda_{i_{0}}^{\dagger}\right)\right)(\alpha) \mathbf{e}_{n}^{i_{0}}, \mathbf{e}_{m}^{i_{0}}\right) \mathscr{\mathscr { C }}_{i} .
\end{aligned}
$$

Next, move the sum over $m$ all the way to the inside-then, mod $\mathbf{0}_{\mathscr{C}_{i}}\left(\boldsymbol{\sigma}_{i_{0}}, \Lambda_{i}+\Lambda_{i_{0}}^{\dagger}: \mathbf{H}\right)$, we get (up to a sign)

$$
\begin{aligned}
D_{*}^{\Sigma_{P_{0}}^{+\prime}} & {\left[\frac{\operatorname{vol}\left(\mathscr{C}_{i^{\prime}}^{\dagger}\right)}{\prod_{\lambda_{k_{0}}^{\dagger} \in \Sigma_{P_{k_{0}}^{\dagger}}^{0}-\Sigma_{P_{k_{0}}^{+\prime}}^{0}\left(?, \lambda_{k_{0}}^{\dagger}\right)}}\right.} \\
& \left.\times\left\langle\mathbf{e}\left(\mathscr{C}_{k_{0}}: \mathbf{H}: ?\right),\left(1_{k_{0} j_{0}}(?), 2_{k_{0} j_{0}}(?)\right)_{\mathscr{C}_{k_{0}}}\right\rangle \mathscr{E}_{1}\right]\left.\right|_{w_{k_{0} J_{0}}^{\dagger^{\prime}} w_{J_{0}}^{0}\left(1-w_{i_{0}}^{\dagger}\right) \Lambda_{t_{0}}^{\dagger}}
\end{aligned}
$$

summed over

$$
n, w_{i_{0}}^{\dagger},\left(j_{0}, w_{j_{0}}^{0}\right),\left(k_{0}, w_{k_{0} j_{0}}^{\dagger \prime}\right)
$$

but this time, at $\Lambda_{k_{0}}^{\dagger}$,

is

$$
\left\{\begin{array}{l}
1_{k_{0} j_{0}}, \\
2_{k_{0} j_{0}}
\end{array}\right.
$$

$$
\left\{\begin{array}{c}
\mathbf{c}\left(P_{k_{0}}\left|A_{k_{0}}: P_{i_{0}}\right| A_{i_{0}}: w_{k_{0} j_{0}}^{\dagger \prime} w_{j_{0}}^{0}: \Lambda_{i_{0}}^{\dagger}\right) \\
\cdot \mathbf{I n d}_{\mathscr{G}_{i_{0}}}^{G}\left(\left(\boldsymbol{\sigma}_{i_{0}}, \Lambda_{i}+\Lambda_{i_{0}}^{\dagger}\right)\right)(\alpha) \mathbf{e}_{n}^{i_{0}}, \\
\mathbf{c}\left(P_{j_{0}}\left|A_{j_{0}}: P_{k_{0}}\right| A_{k_{0}}: w_{k_{0} j_{0}}^{-\dagger}: w_{k_{0} j_{0}}^{\dagger \prime} w_{j_{0}}^{0} \Lambda_{i_{0}}^{\dagger}-\Lambda_{k_{0}}^{\dagger}\right)^{*} \\
\cdot \mathbf{c}\left(P_{j_{0}}\left|A_{j_{0}}: P_{i_{0}}\right| A_{i_{0}}: w_{j_{0}}^{0} w_{i_{0}}^{\dagger}: \Lambda_{i_{0}}^{\dagger}\right) \mathbf{e}_{n}^{i_{0}}
\end{array}\right.
$$


Because

$$
\sum_{n}\left\langle\mathbf{e}\left(\mathscr{C}_{k_{0}}: \mathbf{H}: ?\right),\left(1_{k_{0} j_{0}}(?), 2_{k_{0} j_{0}}(?)\right)_{\mathscr{E}_{k_{0}}}\right\rangle_{\mathscr{C}_{1}}
$$

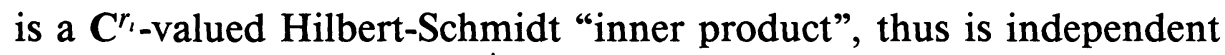
of the orthonormal basis $\left\{\mathbf{e}_{n}^{i_{0}}\right\}$, its value will not change when we make the substitution

$$
\mathbf{e}_{n}^{i_{0}} \rightarrow \mathbf{c}\left(P_{k_{0}}\left|A_{k_{0}}: P_{i_{0}}\right| A_{i_{0}}: w_{k_{0} j_{0}}^{\dagger \prime} w_{j_{0}}^{0}: \Lambda_{i_{0}}^{\dagger}\right)^{-1} \mathbf{e}_{n}^{k_{0}} .
$$

The significance of this replacement is reflected by the intertwining relation

$$
\begin{gathered}
\mathbf{c}\left(P_{k_{0}}\left|A_{k_{0}}: P_{i_{0}}\right| A_{i_{0}}: w_{k_{0} j_{0}}^{\dagger \prime} w_{j_{0}}^{0}: \Lambda_{i_{0}}^{\dagger}\right) \cdot \mathbf{I n d}_{\mathscr{G}_{i_{0}}}^{G}\left(\left(\boldsymbol{\sigma}_{i_{0}}, \Lambda_{i}+\Lambda_{i_{0}}^{\dagger}\right)\right)(\alpha) \\
=\mathbf{I n d}_{\mathscr{E}_{k_{0}}}^{G}\left(\left(\boldsymbol{\sigma}_{k_{0}}, \Lambda_{i}+w_{k_{0} j_{0}}^{\dagger \prime} w_{j_{0}}^{0} \Lambda_{i_{0}}^{\dagger}\right)(\alpha)\right. \\
\cdot \mathbf{c}\left(P_{k_{0}}\left|A_{k_{0}}: P_{i_{0}}\right| A_{i_{0}}: w_{k_{0} j_{0}}^{\dagger \prime} w_{j_{0}}^{0}: \Lambda_{i_{0}}^{\dagger}\right)
\end{gathered}
$$

and the functional equation

$$
\begin{aligned}
\mathbf{c}\left(P_{j_{0}} \mid A_{j_{0}}:\right. & \left.P_{i_{0}} \mid A_{i_{0}}: w_{j_{0}}^{0} w_{i_{0}}^{\dagger}: \Lambda_{i_{0}}^{\dagger}\right) \\
& \cdot \mathbf{c}\left(P_{k_{0}}\left|A_{k_{0}}: P_{i_{0}}\right| A_{i_{0}}: w_{k_{0} j_{0}}^{\dagger \prime} w_{j_{0}}^{0}: \Lambda_{i_{0}}^{\dagger}\right)^{-1} \\
=\mathbf{c} & \left.P_{j_{0}}\left|A_{j_{0}}: P_{k_{0}}\right| A_{k_{0}}:\left(w_{j_{0}}^{0}\right) w_{i_{0}}^{\dagger}\left(w_{j_{0}}^{0}\right)^{-1} w_{k_{0} j_{0}}^{-\dagger}: w_{k_{0} j_{0}}^{\dagger \prime} w_{j_{0}}^{0} \Lambda_{i_{0}}^{\dagger}\right)
\end{aligned}
$$

which, when utilized, mean that at $\Lambda_{k_{0}}^{\dagger}, 1_{k_{0} j_{0}}$ becomes

$$
\operatorname{Ind}_{\mathscr{C}_{k_{0}}}^{G}\left(\left(\boldsymbol{\sigma}_{k_{0}}, \Lambda_{i}+w_{k_{0} j_{0}}^{\dagger \prime} w_{j_{0}}^{0} \Lambda_{i_{0}}^{\dagger}\right)\right)(\alpha) \mathbf{e}_{n}^{k_{0}}
$$

and $2_{k_{0} j_{0}}$ becomes

$$
\begin{aligned}
& \mathbf{c}\left(P_{j_{0}}\left|A_{j_{0}}: P_{k_{0}}\right| A_{k_{0}}: w_{k_{0} j_{0}}^{-\dagger}: w_{k_{0} j_{0}}^{\dagger \prime} w_{j_{0}}^{0} \Lambda_{i_{0}}^{\dagger}-\Lambda_{k_{0}}^{\dagger}\right)^{*} \\
& \quad \times \mathbf{c}\left(P_{j_{0}}\left|A_{j_{0}}: P_{k_{0}}\right| A_{k_{0}}:\left(w_{j_{0}}^{0}\right) w_{i_{0}}^{\dagger}\left(w_{j_{0}}^{0}\right)^{-1} w_{k_{0} j_{0}}^{-\dagger}: w_{k_{0} j_{0}}^{\dagger \prime} w_{j_{0}}^{0} \Lambda_{i_{0}}^{\dagger}\right) \mathbf{e}_{n}^{k_{0}} .
\end{aligned}
$$

To exploit the appearance of

$$
\left\{\begin{array}{l}
w_{k_{0} j_{0}}^{\dagger \prime} w_{j_{0}}^{0}, \\
\left(w_{j_{0}}^{0}\right) w_{i_{0}}^{\dagger}\left(w_{j_{0}}^{0}\right)^{-1},
\end{array}\right.
$$

it will be best to change the sum

$$
\sum_{w_{i_{0}}^{\dagger}} \sum_{\left(j_{0}, w_{j_{0}}^{0}\right)} \sum_{\left(k_{0}, w_{k_{0} j_{0}}^{\dagger \prime}\right)}
$$

to a sum

$$
\sum_{k_{0}} \sum_{w_{k_{0} 0_{0}}^{\dagger}} \sum_{w_{k_{0}}^{\dagger}}
$$


As a first step in this direction, we can certainly assume with no loss of generality that $\Lambda_{i_{0}}^{\dagger}$ is in general position. That being the case,

$$
\Sigma_{P_{k_{0}}^{\dagger \prime}}^{0}=\left\{\lambda_{k_{0}}^{\dagger} \in \Sigma_{P_{k_{0}}^{\dagger}}^{0}: \lambda_{k_{0}}^{\dagger} \perp \operatorname{Im}\left(w_{k_{0} j_{0}}^{\dagger \prime} w_{j_{0}}^{0}\left(1-w_{i_{0}}^{\dagger}\right)\right)\right\}
$$

or still

$$
\Sigma_{P_{j_{0}}^{\dagger \prime}}^{0}=\left\{\lambda_{j_{0}}^{\dagger} \in \Sigma_{P_{j_{0}}^{\dagger}}^{0}: \lambda_{j_{0}}^{\dagger} \perp \operatorname{Im}\left(w_{j_{0}}^{0}\left(1-w_{i_{0}}^{\dagger}\right)\right)\right\}
$$

On the other hand, it is clear that

$$
\lambda_{j_{0}}^{\dagger} \in \Sigma_{P_{j_{0}}^{\dagger}}^{0} \Leftrightarrow \lambda_{j_{0}}^{\dagger} \perp \operatorname{Im}\left(w_{j_{0}}^{0}\left(1-w_{i_{0}}^{\dagger}\right)\right)
$$

i.e.,

$$
\lambda_{j_{0}}^{\dagger} \in \Sigma_{P_{j_{0}}}^{0} \Leftrightarrow\left(w_{j_{0}}^{0}\right) w_{i_{0}}^{\dagger}\left(w_{j_{0}}^{0}\right)^{-1} \lambda_{j_{0}}^{\dagger}=\lambda_{j_{0}}^{\dagger} .
$$

In addition, the domination

$$
P^{\prime} \succeq P_{k_{0}}
$$

produces $P_{k_{0}}^{\dagger \prime}$, thereby determining an association class in $M^{\prime}$ containing $P_{j_{0}}^{\dagger \prime}$ as a fixed representative, the pair $\left(j_{0}, w_{j_{0}}^{0}\right)$ serving as a fixed "base-point" choice. Letting

$$
w_{j_{0}}^{\dagger}=\left(w_{j_{0}}^{0}\right) w_{i_{0}}^{\dagger}\left(w_{j_{0}}^{0}\right)^{-1}
$$

we have

$$
\left\{\begin{array}{l}
w_{k_{0}}^{\dagger}=w_{k_{0} j_{0}}^{\dagger \prime} w_{j_{0}}^{\dagger} w_{k_{0} j_{0}}^{-\dagger \prime}, \\
w_{k_{0} i_{0}}^{\dagger}=w_{k_{0} j_{0}}^{\dagger \prime} w_{j_{0}}^{0},
\end{array}\right.
$$

with dependencies

$$
\left\{\begin{array}{l}
j_{0}=j_{0}\left(w_{k_{0}}^{\dagger}\right) \\
w_{k_{0} j_{0}}^{\dagger \prime}=w_{k_{0} j_{0}}^{\dagger \prime}\left(w_{k_{0}}^{\dagger}, w_{k_{0} i_{0}}^{\dagger}\right)
\end{array}\right.
$$

Relative to $w_{k_{0}}^{\dagger}$, put

$$
\Sigma_{P_{k_{0}}^{\dagger}}^{0}\left(w_{k_{0}}^{\dagger}\right)=\left\{\lambda_{k_{0}}^{\dagger} \in \Sigma_{P_{k_{0}}^{\dagger}}^{0}: w_{k_{0}}^{\dagger} \lambda_{k_{0}}^{\dagger}=\lambda_{k_{0}}^{\dagger}\right\}
$$

Then, apart from the error term, our integrand is seen to be $(-1)^{l_{0}^{+}}$ times the sum

$$
\sum_{n} \sum_{k_{0}} \sum_{w_{i t o}^{t}} \sum_{w_{t o}^{t}}
$$


of the

$$
\begin{aligned}
& (-1)^{\#\left(\Sigma_{p_{k_{0}}^{\dagger \prime}}^{0}\left(w_{k_{0}}^{\dagger}\right)\right)} \cdot D_{*}^{\Sigma_{P_{k_{0}}^{\dagger \prime}}^{0}\left(w_{k_{0}}^{\dagger}\right)}\left[\frac{\operatorname{vol}\left(\mathscr{C}_{i^{\prime}}^{\dagger}\right)}{\prod_{\lambda_{k_{0}}^{\dagger} \in \Sigma_{P_{k_{0}}^{\dagger}}^{0}-\Sigma_{P_{k_{0}}^{\dagger}}^{0}\left(w_{k_{0}}^{\dagger}\right)}\left(?, \lambda_{k_{0}}^{\dagger}\right)}\right. \\
& \left.\times\left\langle\mathbf{e}\left(\mathscr{C}_{k_{0}}: \mathbf{H}: ?\right),\left(1_{k_{0} j_{0}}(?), 2_{k_{0} j_{0}}(?)\right)_{\mathscr{C}_{k_{0}}}\right\rangle_{\mathscr{C}_{1}}\right]\left.\right|_{\left(1-w_{k_{0}}^{\dagger}\right) w_{k_{0} i_{0}}^{\dagger} \Lambda_{t_{0}}^{\dagger}} \\
& \left\{\begin{array}{l}
1_{k_{0} j_{0}}, \\
2_{k_{0} j_{0}}
\end{array}\right.
\end{aligned}
$$

at $\Lambda_{k_{0}}^{\dagger}$ now being given by

$$
\operatorname{Ind}_{\mathscr{E}_{k_{0}}}^{G}\left(\left(\boldsymbol{\sigma}_{k_{0}}, \Lambda_{i}+w_{k_{0} i_{0}}^{\dagger} \Lambda_{i_{0}}^{\dagger}\right)\right)(\alpha) \mathbf{e}_{n}^{k_{0}}
$$

and

$$
\begin{aligned}
& \mathbf{c}\left(P_{j_{0}}\left|A_{j_{0}}: P_{k_{0}}\right| A_{k_{0}}: w_{k_{0} j_{0}}^{-\dagger}: w_{k_{0} i_{0}}^{\dagger} \Lambda_{i_{0}}^{\dagger}-\Lambda_{k_{0}}^{\dagger}\right)^{*} \\
& \quad \cdot \mathbf{c}\left(P_{j_{0}}\left|A_{j_{0}}: P_{k_{0}}\right| A_{k_{0}}: w_{k_{0} j_{0}}^{\dagger \dagger} w_{k_{0}}^{\dagger}: w_{k_{0} i_{0}}^{\dagger} \Lambda_{i_{0}}^{\dagger}\right) \mathbf{e}_{n}^{k_{0}},
\end{aligned}
$$

respectively. Finally, transferring the c-functions to the left,

$$
\sum_{n}\left\langle\mathbf{e}\left(\mathscr{C}_{k_{0}}: \mathbf{H}: ?\right),\left(1_{k_{0} j_{0}}(?), 2_{k_{0} j_{0}}(?)\right)_{\mathscr{C}_{k_{0}}}\right\rangle_{\mathscr{C}_{l}}
$$

reduces to

$$
\left\langle\mathbf{e}\left(\mathscr{C}_{k_{0}}: \mathbf{H}: ?\right), \operatorname{tr}_{\mathscr{C}_{k_{0}}}\left(\mathbf{T}_{k_{0} j_{0}}(\alpha: ?)\right)\right\rangle_{\mathscr{C}_{i}},
$$

where $\mathbf{T}_{k_{0} j_{0}}(\alpha: ?)$ is the consecutive composition of

$$
\left\{\begin{array}{l}
\mathbf{c}\left(P_{j_{0}}\left|A_{j_{0}}: P_{k_{0}}\right| A_{k_{0}}: w_{k_{0} j_{0}}^{-\dagger} w_{k_{0}}^{\dagger}: w_{k_{0} i_{0}}^{\dagger} \Lambda_{i_{0}}^{\dagger}\right)^{*} \\
\mathbf{c}\left(P_{j_{0}}\left|A_{j_{0}}: P_{k_{0}}\right| A_{k_{0}}: w_{k_{0} j_{0}}^{-\dagger}: w_{k_{0} i_{0}}^{\dagger} \Lambda_{i_{0}}^{\dagger}-?\right)
\end{array}\right.
$$

and

$$
\operatorname{Ind}_{\mathscr{C}_{k_{0}}}^{G}\left(\left(\boldsymbol{\sigma}_{k_{0}}, \Lambda_{i}+w_{k_{0} i_{0}}^{\dagger} \Lambda_{i_{0}}^{\dagger}\right)\right)(\alpha)
$$

This is a good point at which to pause and prepare a statement of recapitulation. Let us agree to write

$$
\mathbf{I}_{\alpha}\left(\mathscr{C}_{i}: \mathscr{\theta}_{i_{0}}, \Lambda_{i}+\Lambda_{i_{0}}^{\dagger}: \mathbf{H}\right)
$$


for

$$
\begin{aligned}
& \left\langle\sum_{m, n} \mathbf{C}_{m n}\left(\alpha: \boldsymbol{O}_{i_{0}}, \Lambda_{i}+\Lambda_{i_{0}}^{\dagger}\right) \mathscr{C}_{\imath},\right. \\
& \left(\mathbf{Q}_{\mathscr{C}_{\imath}}^{\mathbf{H}} \mathbf{E}\left(\mathscr{C}_{i}: P_{i_{0}} \mid A_{i_{0}}: \mathbf{e}_{m}^{i_{0}}: \Lambda_{i_{0}}^{\dagger}: ?\right)\right. \\
& \left.\left.\quad \mathbf{Q}_{\mathscr{C}_{\imath}}^{\mathbf{H}} \mathbf{E}\left(\mathscr{C}_{i}: P_{i_{0}} \mid A_{i_{0}}: \mathbf{e}_{n}^{i_{0}}: \Lambda_{i_{0}}^{\dagger}: ?\right)\right)_{\mathscr{C}_{l}}\right\rangle_{\mathscr{C}_{l}}
\end{aligned}
$$

so that

$$
\operatorname{tr}_{\mathscr{C}_{i}}\left(\mathbf{Q}_{\mathscr{C}_{i}}^{\mathbf{H}} \circ \mathbf{I n d}_{\mathscr{C}_{l}}^{G}\left(\mathbf{L}_{\mathscr{C}_{i} / \Gamma_{\mathscr{C}_{l}}}^{\text {con }} \otimes \Lambda_{i} \otimes 1\right)(\alpha) \circ \mathbf{Q}_{\mathscr{C}_{l}}^{\mathbf{H}}\right)
$$

is equal to

$$
\begin{aligned}
\sum_{\mathscr{E}_{i_{0}}: \mathscr{E}_{i_{0}} \prec \mathscr{E}_{i}} & \sum_{\boldsymbol{O}_{i_{0}}} \frac{1}{(2 \pi)^{l_{0}^{\dagger}}} \cdot \frac{1}{*\left(\mathscr{C}_{i_{0}}^{\dagger}\right)} \\
& \times \int_{\operatorname{Re}\left(\Lambda_{i_{0}}^{\dagger}\right)=0} \mathbf{I}_{\alpha}\left(\mathscr{C}_{i}: \boldsymbol{\Theta}_{i_{0}}, \Lambda_{i}+\Lambda_{i_{0}}^{\dagger}: \mathbf{H}\right)\left|d \Lambda_{i_{0}}^{\dagger}\right|
\end{aligned}
$$

Noting that (cf. TES, p. 361)

$$
\mathbf{0}_{\mathscr{C}_{i}}\left(\boldsymbol{\sigma}_{i_{0}}, \Lambda_{i}+\Lambda_{i_{0}}^{\dagger}: \mathbf{H}\right)=\mathbf{o}_{\mathscr{C}_{i}}\left(\boldsymbol{\sigma}_{k_{0}}, \Lambda_{i}+w_{k_{0} i_{0}}^{\dagger} \Lambda_{i_{0}}^{\dagger}: \mathbf{H}\right),
$$

the preceding manipulations can be summarized as follows:

Proposition 9.1. Let $\alpha$ be a $K$-central, $K$-finite element of $C_{c}^{\infty}(G)-$ then

$$
\begin{aligned}
& \mathbf{I}_{\alpha}\left(\mathscr{C}_{i}: \boldsymbol{\sigma}_{i_{0}}, \Lambda_{i}+\Lambda_{i_{0}}^{\dagger}: \mathbf{H}\right) \\
& =\mathbf{0}_{\mathscr{C}_{i}}\left(\boldsymbol{\sigma}_{i_{0}}, \Lambda_{i}+\Lambda_{i_{0}}^{\dagger}: \mathbf{H}\right) \\
& +(-1)^{l_{0}^{\dagger}} \cdot \sum_{k_{0}} \sum_{w_{k_{0} 0_{0}}^{\dagger}} \sum_{w_{k_{0}}^{\dagger}}(-1)^{\#\left(\Sigma_{P_{k_{0}}^{\dagger}}^{0}\left(w_{k_{0}}^{\dagger}\right)\right)} \\
& \cdot D_{*}^{\Sigma_{P_{k_{0}}^{\dagger \prime}}^{0}\left(w_{k_{0}}^{\dagger}\right)}\left[\frac{\operatorname{vol}\left(\mathscr{C}_{i^{\prime}}^{\dagger}\right)}{\prod_{\lambda_{k_{0}}^{\dagger} \in \Sigma_{P_{k_{0}}^{\dagger}}^{0}-\Sigma_{P_{k_{0}}^{\dagger}}^{0}\left(w_{k_{0}}^{\dagger}\right)}\left(?, \lambda_{k_{0}}^{\dagger}\right)}\right. \\
& \left.\times\left\langle\mathbf{e}\left(\mathscr{C}_{k_{0}}: \mathbf{H}: ?\right), \operatorname{tr}_{\mathscr{C}_{k_{0}}}\left(\mathbf{T}_{k_{0} j_{0}}(\alpha: ?)\right)\right\rangle_{\mathscr{C}_{1}}\right]\left.\right|_{\left(1-w_{k_{0}}^{\dagger}\right) w_{k_{0} t_{0}}^{\dagger} \Lambda_{t_{0}}^{\dagger}}
\end{aligned}
$$

[Note: $\Lambda_{i_{0}}^{\dagger}$ is in general position.] 
Ultimately, we must study

$$
\frac{1}{(2 \pi)^{l_{i}}} \cdot \int_{\operatorname{Re}\left(\Lambda_{i}\right)=0} \cdots\left|d \Lambda_{i}\right|
$$

... being

$$
\begin{aligned}
& \sum_{\mathscr{E}_{1_{0}}: \mathscr{E}_{i_{0}}<\mathscr{E}_{1}} \sum_{\boldsymbol{\theta}_{i_{0}}} \frac{1}{(2 \pi)^{l_{0}^{\dagger}}} \cdot \frac{1}{*\left(\mathscr{C}_{i_{0}}^{\dagger}\right)} \\
& \quad \times \int_{\operatorname{Re}\left(\Lambda_{i_{0}}^{\dagger}\right)=0} \mathbf{I}_{\alpha}\left(\mathscr{C}_{i}: \boldsymbol{\theta}_{i_{0}}, \Lambda_{i}+\Lambda_{i_{0}}^{\dagger}: \mathbf{H}\right)\left|d \Lambda_{i_{0}}^{\dagger}\right|
\end{aligned}
$$

To justify our calculations, a lemma of convergence will be needed.

LEMMA 9.2. Let $\alpha$ be a $K$-central, $K$-finite element of $C_{c}^{\infty}(G)$-then $\int_{\operatorname{Re}\left(\Lambda_{i}\right)=0} \sum_{\boldsymbol{O}_{i_{0}}} \int_{\operatorname{Re}\left(\Lambda_{i_{0}}^{\dagger}\right)=0}\left\|\mathbf{I}_{\alpha}\left(\mathscr{C}_{i}: \boldsymbol{\sigma}_{i_{0}}, \Lambda_{i}+\Lambda_{i_{0}}^{\dagger}: \mathbf{H}\right)\right\|\left|d \Lambda_{i_{0}}^{\dagger}\right|\left|d \Lambda_{i}\right|<+\infty$.

Proof. Suppose first that

$$
\alpha=\bar{\chi}_{F} *\left(\mu * \mu^{*}\right)_{K} * \bar{\chi}_{F}
$$

for some $\mu \in C_{c}^{\infty}(G)$. Let $U$ be a unitary representation of $G$-then it is easy to verify that

$$
U(\alpha)=U\left(\bar{\chi}_{F} *\left(\mu * \mu^{*}\right)_{K} * \bar{\chi}_{F}\right)
$$

is a positive definite operator. In this case, therefore, the entries in the integrand associated with the representatives of $\mathscr{C}_{i}$ are themselves positive; hence, after summing over $\mathscr{C}_{i_{0}}$, there arises the majorant

$$
\left\|\operatorname{tr}_{\mathscr{C}_{i}}\left(\mathbf{Q}^{\mathbf{I}_{\mathscr{Z}_{i}}(\mathbf{H})} \circ \mathbf{L}_{\mathscr{E}_{i} / \Gamma_{\mathscr{C}_{i}}}^{\text {con }}\left(\alpha^{\mathscr{C}_{i}}\right) \circ \mathbf{Q}^{\mathbf{I}_{\mathscr{Z}_{i}}(\mathbf{H})}\right)\right\|
$$

a finite quantity. In general, the factorization theorem of Dixmier and Malliavin allows one to write every $\alpha \in C_{c}^{\infty}(G)$ as a finite sum of convolution products $\mu_{i} * \nu_{i}$, where $\mu_{i}, \nu_{i} \in C_{c}^{\infty}(G)$ (cf. TES, p. 20). Furthermore,

$$
\begin{aligned}
\mu_{i} * \nu_{i}=\frac{1}{2}(\sqrt{-1} & \left(\mu_{i}+\sqrt{-1} \nu_{i}^{*}\right) *\left(\mu_{i}+\sqrt{-1} \nu_{i}^{*}\right)^{*} \\
+ & \left(\mu_{i}+\nu_{i}^{*}\right) *\left(\mu_{i}+\nu_{i}^{*}\right)^{*} \\
& \left.-(\sqrt{-1}+1)\left(\mu_{i} * \mu_{i}^{*}+\nu_{i}^{*} * \nu_{i}\right)\right) .
\end{aligned}
$$

Consequently, a $K$-central, $K$-finite $\alpha \in C_{c}^{\infty}(G)$ always admits a decomposition of the form

$$
\sum_{\mu} C_{\mu} \cdot \bar{\chi}_{F} *\left(\mu * \mu^{*}\right)_{K} * \bar{\chi}_{F} \quad\left(C_{\mu} \in \mathbf{C}\right)
$$

from which the lemma. 
Unfortunately, this lemma alone does not suffice to completely ensure the validity of the upcoming manipulations. Therefore, in order to proceed with a minimal amount of contrivance, what we shall do is make an assumption (on the behavior of the c-functions).

Assumption c. For each $K$-type $\delta$, there exist positive constants $\varepsilon$, $M$, and $N$ with the following property:

$$
\begin{gathered}
\forall i_{0}, \forall \boldsymbol{\Theta}_{i_{0}}, \forall \Lambda_{i_{0}} \in \sqrt{-1} \check{\mathfrak{a}}_{i_{0}}, \forall \lambda_{i_{0}} \in \check{\mathfrak{a}}_{i_{0}}, \forall w_{j_{0} i_{0}} \in W\left(A_{j_{0}}, A_{i_{0}}\right): \\
\left\|\mathbf{c}\left(P_{j_{0}}\left|A_{j_{0}}: P_{i_{0}}\right| A_{i_{0}}: w_{j_{0} i_{0}}: \Lambda_{i_{0}}+\lambda_{i_{0}}\right)\right\|_{\mathrm{OP}} \\
<M \cdot\left(1+\left\|\boldsymbol{\Theta}_{i_{0}}\right\|\right)^{N} \cdot\left(1+\left\|\Lambda_{i_{0}}\right\|\right)^{N}
\end{gathered}
$$

provided

$$
\left\|\lambda_{i_{0}}\right\|<\varepsilon \cdot\left(1+\left\|\Theta_{i_{0}}\right\|\right)^{-N} \cdot\left(1+\left\|\Lambda_{i_{0}}\right\|\right)^{-N} .
$$

[Note: We have put

$$
\left\|\boldsymbol{\theta}_{i_{0}}\right\|=\left|\chi\left(\omega_{i_{0}}\right)\right| \quad\left(\chi \in \boldsymbol{\theta}_{i_{0}}\right)
$$

$\omega_{i_{0}}$ the Casimir operator associated with $\mathscr{C}_{i_{0}}$.]

The nature of this assumption will be examined in the Appendix. At the moment, we need only make a few simple observations.

REMARKs. (1) The positive constants $\varepsilon, M$, and $N$ are uniform in the data except, perhaps, for the $K$-type $\delta$. The latter point is not a problem since we are dealing throughout with $K$-finite functions.

(2) Our assumption is hereditary in the sense that if it holds for the c-functions on $G$, then it holds for the c-functions on $M(P=M \cdot A \cdot N)$. Indeed, thanks to the lemma of descent, then c-functions on $M$ "are" c-functions on $G$.

(3) Since

$$
\mathbf{c}=\mathbf{c}_{\text {cus }} \oplus \mathbf{c}_{\text {res }}
$$

the estimate of the assumption holds for both $\mathbf{c}_{\text {cus }}$ and $\mathbf{c}_{\text {res. }}$.

(4) Let $D$ be a constant coefficient differential operator-then the Cauchy integral formula implies that

$$
\left\|D \mathbf{c}\left(P_{j_{0}}\left|A_{j_{0}}: P_{i_{0}}\right| A_{i_{0}}: w_{j_{0} i_{0}}: \Lambda_{i_{0}}+\lambda_{i_{0}}\right)\right\|_{\mathrm{OP}}
$$

admits a bound of the same general form as

$$
\left\|\mathbf{c}\left(P_{j_{0}}\left|A_{j_{0}}: P_{i_{0}}\right| A_{i_{0}}: w_{j_{0} i_{0}}: \Lambda_{i_{0}}+\lambda_{i_{0}}\right)\right\|_{\mathrm{OP}}
$$

itself with, of course, different positive constants $\varepsilon_{D}, M_{D}$, and $N_{D}$.

(5) The assumption automatically forces the traceability of $L_{G / \Gamma}^{\text {dis }}(\alpha)$ for any $K$-finite $\alpha$ in $C_{c}^{\infty}(G)$ (or $\mathscr{C}^{1}(G)$ ) and, analogously, with $G \rightarrow$ $M$. The verification may be found in the Appendix. 
Lemma 9.3. Let $\alpha \in C_{c}^{\infty}(G)$ be $K$-central and $K$-finite-then

$$
\tau\left(\text { Ind }_{M_{i_{0}} \cdot A_{t_{0}} \cdot N_{t_{0}}}\left(\left(\boldsymbol{\sigma}_{i_{0}}, \Lambda_{i_{0}}\right)\right)(\alpha)\right)
$$

is a rapidly decreasing function of

$$
\left\{\begin{array}{l}
\boldsymbol{\sigma}_{i_{0}}, \\
\Lambda_{i_{0}}=\Lambda_{i}+\Lambda_{i_{0}}^{\dagger} .
\end{array}\right.
$$

Proof. We can assume without loss of generality that $\alpha$ transforms according to a fixed $K$-type $\delta$, say, so that

$$
\text { Ind }_{M_{i_{0}} \cdot A_{t_{0}} \cdot N_{i_{0}}}^{G}\left(\left(\boldsymbol{\sigma}_{i_{0}}, \Lambda_{i_{0}}\right)\right)(\alpha): \mathscr{E}\left(\boldsymbol{\delta}, \boldsymbol{\theta}_{i_{0}}\right) \rightarrow \mathscr{E}\left(\boldsymbol{\delta}, \boldsymbol{\theta}_{i_{0}}\right) \text {. }
$$

The theory of Eisenstein systems provides a decomposition of $\mathscr{E}\left(\delta, \boldsymbol{\theta}_{i_{0}}\right)$ into a finite orthogonal sum of other spaces, the explicit description of which is part of the residue taking process (cf. TES, pp. 302-303). Each of these spaces is preserved by

$$
\operatorname{Ind}_{M_{t_{0}} \cdot A_{t_{0}} \cdot N_{t_{0}}}^{G}\left(\left(\boldsymbol{\sigma}_{i_{0}}, \Lambda_{i_{0}}\right)\right)(\alpha),
$$

the action of $\omega$ then being explicitly computable in that if $X_{i_{0}}$ is an ambient normal translation, then

$$
\operatorname{Ind}_{M_{t_{0}} \cdot A_{t_{0}} \cdot N_{t_{0}}}^{G}\left(\left(\boldsymbol{\sigma}_{i_{0}}, \Lambda_{i_{0}}\right)\right)(\omega \alpha)
$$

is

$$
\left(\left\|\boldsymbol{\sigma}_{i_{0}}\right\|+\left(\Lambda_{i_{0}}+X_{i_{0}}, \Lambda_{i_{0}}+X_{i_{0}}\right)-\left\|\rho_{i_{0}}\right\|^{2}\right)
$$

times

$$
\operatorname{Ind}_{M_{t_{0}} \cdot A_{t_{0}} \cdot N_{i_{0}}}^{G}\left(\left(\boldsymbol{\theta}_{i_{0}}, \Lambda_{i_{0}}\right)\right)(\alpha) .
$$

Because the norms of the various possible $X_{i_{0}}$ 's admit an absolute upper bound (cf. TES, p. 346), by working with $\omega^{N}$ as opposed to $\omega$, the moduli of the "eigenvalues" are of the order

$$
\left(1+\left\|\mathcal{O}_{i_{0}}\right\|+\left\|\Lambda_{i_{0}}\right\|^{2}\right)^{N} \quad(N \gg 0) .
$$

Consequently,

$$
\| \text { Ind }_{M_{i_{0}} \cdot A_{t_{0}} \cdot N_{t_{0}}}^{G}\left(\left(\boldsymbol{\sigma}_{i_{0}}, \Lambda_{i_{0}}\right)\right)(\alpha) \|_{\mathrm{OP}}
$$

is a rapidly decreasing function of

$$
\left\{\begin{array}{l}
\boldsymbol{\sigma}_{i_{0}}, \\
\Lambda_{i_{0}}=\Lambda_{i}+\Lambda_{i_{0}}^{\dagger} .
\end{array}\right.
$$

However,

$$
\begin{aligned}
& \tau\left(\text { Ind }_{M_{t_{0}} \cdot A_{t_{0}} \cdot N_{t_{0}}}^{G}\left(\left(\boldsymbol{\sigma}_{i_{0}}, \Lambda_{i_{0}}\right)\right)(\alpha)\right) \\
& \quad \leq \operatorname{dim}\left(\mathscr{E}\left(\delta, \boldsymbol{\theta}_{i_{0}}\right)\right) \cdot \| \text { Ind }_{M_{t_{0}} \cdot A_{t_{0}} \cdot N_{t_{0}}}^{G}\left(\left(\boldsymbol{\sigma}_{i_{0}}, \Lambda_{i_{0}}\right)\right)(\alpha) \|_{\mathrm{OP}}
\end{aligned}
$$


and, from traceability on the discrete spectrum (cf. supra),

$$
\sum_{\boldsymbol{\theta}_{i_{0}}} \operatorname{dim}\left(\mathscr{E}\left(\delta, \boldsymbol{\theta}_{i_{0}}\right)\right) \cdot\left(1+\left\|\boldsymbol{\theta}_{i_{0}}\right\|\right)^{-N}<+\infty \quad(N \gg 0) .
$$

Therefore

$$
\operatorname{dim}\left(\mathscr{E}\left(\delta, \boldsymbol{\theta}_{i_{0}}\right)\right)
$$

is a slowly increasing function of $\mathscr{\theta}_{i_{0}}$. The truth of our lemma is now evident.

Taking into account the formula for $\mathbf{I}_{\alpha}$ derived above and the fact that

$$
\int_{\operatorname{Re}\left(\Lambda_{i}\right)=0} \int_{\operatorname{Re}\left(\Lambda_{t_{0}}^{\dagger}\right)=0}=\int_{\operatorname{Re}\left(\Lambda_{t_{0}}\right)=0},
$$

we can use Lemmas 9.2 and 9.3, together with Proposition 4.6, to break up the integral-series

$$
\int_{\operatorname{Re}\left(\Lambda_{t}\right)=0} \sum_{\boldsymbol{\theta}_{i_{0}}} \int_{\operatorname{Re}\left(\Lambda_{i_{0}}^{\dagger}\right)=0} \mathbf{I}_{\alpha}\left(\mathscr{C}_{i}: \boldsymbol{\sigma}_{i_{0}}, \Lambda_{i}+\Lambda_{i_{0}}^{\dagger}: \mathbf{H}\right)\left|d \Lambda_{i_{0}}^{\dagger}\right|\left|d \Lambda_{i}\right|
$$

as the sum of two other integral-series, namely

$$
\begin{aligned}
& \sum_{\boldsymbol{\theta}_{i_{0}}} \int_{\operatorname{Re}\left(\Lambda_{i_{0}}\right)=0} \mathbf{o}_{\mathscr{C}_{\imath}}\left(\boldsymbol{\sigma}_{i_{0}}, \Lambda_{i_{0}}: \mathbf{H}\right)\left|d \Lambda_{i_{0}}\right| \\
& +(-1)^{l_{0}^{\dagger}} \cdot \sum_{\boldsymbol{O}_{i_{0}}} \int_{\operatorname{Re}\left(\Lambda_{i_{0}}\right)=0} \sum_{k_{0}} \sum_{w_{k_{0} 0_{0}}^{\dagger}} \sum_{w_{k_{0}}^{\dagger}}(-1)^{\#\left(\Sigma_{k_{0}}^{0}\left(w_{k_{0}}^{\dagger}\right)\right)} \\
& \cdot D_{*}^{\Sigma_{k_{0}}^{\dagger}\left(w_{k_{0}}^{\dagger}\right)}\left[\frac{\operatorname{vol}\left(\mathscr{C}_{i^{\prime}}^{\dagger}\right)}{\prod_{\lambda_{k_{0}}^{\dagger} \in \Sigma_{P_{k_{0}}^{\dagger}}^{0}-\Sigma_{P^{\prime} k_{0}}^{0}\left(w_{k_{0}}^{\dagger}\right)}\left(?, \lambda_{k_{0}}^{\dagger}\right)}\right. \\
& \left.\times\left\langle\mathbf{e}\left(\mathscr{C}_{k_{0}}: \mathbf{H}: ?\right), \operatorname{tr}_{\mathscr{C}_{k_{0}}}\left(\mathbf{T}_{k_{0} j_{0}}(\alpha: ?)\right)\right\rangle_{\mathscr{C}_{1}}\right]\left.\right|_{\left(1-w_{k_{0}}^{\dagger}\right) w_{k_{0} t^{\prime}}^{\dagger} \Lambda_{t_{0}}}\left|d \Lambda_{i_{0}}\right|,
\end{aligned}
$$

both of which are absolutely convergent. It is to be noted that the definition of $\mathbf{T}_{k_{0} j_{0}}(\alpha: ?)$ must then be altered slightly, being in this context the consecutive composition of

$$
\left\{\begin{array}{l}
\mathbf{c}\left(P_{j_{0}}\left|A_{j_{0}}: P_{k_{0}}\right| A_{k_{0}}: w_{k_{0} j_{0}}^{-\dagger \prime} w_{k_{0}}^{\dagger}:-w_{k_{0} i_{0}}^{\dagger} \bar{\Lambda}_{i_{0}}\right)^{*} \\
\mathbf{c}\left(P_{j_{0}}\left|A_{j_{0}}: P_{k_{0}}\right| A_{k_{0}}: w_{k_{0} j_{0}}^{\dagger \dagger} w_{k_{0} j_{0}}: w_{k_{0} i_{0}}^{\dagger} \Lambda_{i_{0}}-?\right)
\end{array}\right.
$$

and

$$
\operatorname{Ind}_{\mathscr{E}_{k_{0}}}^{G}\left(\left(\boldsymbol{\sigma}_{k_{0}}, w_{k_{0} i_{0}}^{\dagger} \Lambda_{i_{0}}\right)\right)(\alpha)
$$


The error term is of no immediate concern, thus will be set aside. To discuss what is left, it will be necessary to pass into the "complex domain" and change the integral over $\operatorname{Re}\left(\Lambda_{i_{0}}\right)=0$ to a contour integral over a region inside that encompassed by Assumption c.

Let us start by isolating the main idea. For this purpose, fix a real unit vector $\lambda_{i_{0}}$ in general position. Working with some specified $\theta_{i_{0}}$, suppose that $\phi$ is holomorphic and rapidly decreasing on

$$
\begin{aligned}
\left\{z \lambda_{i_{0}}+\Lambda_{i_{0}} \in \mathbf{C} \lambda_{i_{0}}+\right. & \sqrt{-1} \lambda_{i_{0}}^{\perp}: \\
& \left.|\operatorname{Re}(z)|<\varepsilon\left(\boldsymbol{\Theta}_{i_{0}}\right)\left(1+(\operatorname{Im}(z))^{2}+\left\|\Lambda_{i_{0}}\right\|^{2}\right)^{-N}\right\},
\end{aligned}
$$

$\varepsilon\left(\boldsymbol{\sigma}_{i_{0}}\right)$ sufficiently small-then

$$
\begin{aligned}
& \int_{\operatorname{Re}\left(\Lambda_{i_{0}}\right)=0} \phi\left(\Lambda_{i_{0}}\right)\left|d \Lambda_{i_{0}}\right| \\
& =\frac{1}{\sqrt{-1}} \cdot \int_{\operatorname{Re}\left(\Lambda_{i_{0}}\right)=0 \& \Lambda_{i_{0}} \perp \lambda_{i_{0}}} \int_{-\sqrt{-1} \infty}^{+\sqrt{-1} \infty} \phi\left(z \lambda_{i_{0}}+\Lambda_{i_{0}}\right) d z\left|d \Lambda_{i_{0}}\right| .
\end{aligned}
$$

With $\Lambda_{i_{0}}$ subject to the conditions

$$
\left\{\begin{array}{l}
\operatorname{Re}\left(\Lambda_{i_{0}}\right)=0, \\
\Lambda_{i_{0}} \perp \lambda_{i_{0}}
\end{array}\right.
$$

the integral

$$
\int_{-\sqrt{-1} \infty}^{+\sqrt{-1} \infty} \phi\left(z \lambda_{i_{0}}+\Lambda_{i_{0}}\right) d z
$$

may, thanks to our assumptions on $\phi$, be replaced by the integral

$$
\int_{\mathfrak{C}\left(\Lambda_{i_{0}}\right)} \phi\left(z \lambda_{i_{0}}+\Lambda_{i_{0}}\right) d z
$$

where $\mathfrak{C}\left(\Lambda_{i_{0}}\right)$ is the "witch-curve"

$$
\operatorname{Re}(z)=\frac{1}{2} \cdot \varepsilon\left(\boldsymbol{\sigma}_{i_{0}}\right)\left(1+(\operatorname{Im}(z))^{2}+\left\|\Lambda_{i_{0}}\right\|^{2}\right)^{-N} .
$$

We cannot quite apply these remarks to our situation since the integrand is known to be Schwartz only on $\operatorname{Re}\left(\Lambda_{i_{0}}\right)=0$. But it is at least holomorphic in the region into which we wish to shift the contour, meaning that we need only examine the individual terms in a neighborhood of infinity along the witch. More precisely, if

$$
\left\{\begin{array}{l}
\operatorname{Re}\left(\Lambda_{i_{0}}\right)=0, \\
\Lambda_{i_{0}} \perp \lambda_{i_{0}},
\end{array}\right.
$$

then by Cauchy's theorem, the integral from $-\sqrt{-1} \infty$ to $+\sqrt{-1} \infty$ can be replaced by an integral along a contour $\mathfrak{C}\left(\Lambda_{i_{0}}: T\right)$ which follows 
the imaginary axis from $-\sqrt{-1} \infty$ to $-\sqrt{-1} T$, goes along the witch to $+\sqrt{-1} T$, and, back on the imaginary axis again, heads out to $+\sqrt{-1} \infty$. Because $\lambda_{i_{0}}$ is in general position, the root denominators will be nonzero if $T \gg 0$, that is,

$$
\begin{gathered}
T \gg 0 \Rightarrow \forall \lambda_{k_{0}}^{\dagger} \in \Sigma_{P_{k_{0}}^{\dagger}}^{0}-\Sigma_{P_{k_{0}}^{\dagger}}^{0}\left(w_{k_{0}}^{\dagger}\right), \\
\left(\left(1-w_{k_{0}}^{\dagger}\right) w_{k_{0} i_{0}}^{\dagger}\left(z \lambda_{i_{0}}+\Lambda_{i_{0}}\right), \lambda_{k_{0}}^{\dagger}\right) \neq 0 \quad\left(z \in \mathfrak{C}\left(\Lambda_{i_{0}}: T\right)\right) .
\end{gathered}
$$

This in turn allows us to consider each term separately. Indeed, on the two tails of the region determined by $\pm \sqrt{-1} T$, the root denominators blow up, forcing the requisite decay at infinity (the Schwartz substitute). It is therefore permissible to pass from $\mathfrak{C}\left(\Lambda_{i_{0}}: T\right)$ to $\mathfrak{C}\left(\Lambda_{i_{0}}\right)$ term-by-term giving

$$
\begin{aligned}
& \int_{\operatorname{Re}\left(\Lambda_{t_{0}}\right)=0} \sum_{k_{0}} \sum_{w_{k_{0} i_{0}}^{\dagger}} \sum_{w_{k_{0}}^{\dagger}}(-1)^{\#\left(\Sigma_{p_{k_{0}}^{\dagger}}^{0}\left(w_{k_{0}}^{\dagger}\right)\right)} \\
& \cdot D_{*}^{\Sigma_{k_{0}}^{\dagger}\left(w_{k_{0}}^{\dagger}\right)}\left[\frac{\operatorname{vol}\left(\mathscr{C}_{i^{\prime}}^{\dagger}\right)}{\prod_{\lambda_{k_{0}}^{\dagger} \in \Sigma_{P_{k_{0}}^{\dagger}}^{0}-\Sigma_{P_{k_{0}}^{\dagger}}^{0}\left(w_{k_{0}}^{\dagger}\right)}\left(?, \lambda_{k_{0}}^{\dagger}\right)}\right. \\
& \left.\times\left\langle\mathbf{e}\left(\mathscr{C}_{k_{0}}: \mathbf{H}: ?\right), \mathbf{t r}_{\mathscr{C}_{k_{0}}}\left(\mathbf{T}_{k_{0} j_{0}}(\alpha: ?)\right)\right\rangle_{\mathscr{C}_{i}}\right]\left.\right|_{\left(1-w_{k_{0}}^{\dagger}\right) w_{k_{0} i_{0}}^{\dagger} \Lambda_{i_{0}}}\left|d \Lambda_{i_{0}}\right|, \\
& =\frac{1}{\sqrt{-1}} \cdot \sum_{k_{0}} \sum_{w_{k_{0} t_{0}}^{\dagger}} \sum_{w_{k_{0}}^{\dagger}} \int_{\operatorname{Re}\left(\Lambda_{t_{0}}\right)=0 \& \Lambda_{i_{0}} \perp \lambda_{i_{0}}} \int_{\mathfrak{C}\left(\Lambda_{t_{0}}\right)}(-1)^{\#\left(\Sigma_{P_{k_{0}}^{\dagger}}^{0}\left(w_{k_{0}}^{\dagger}\right)\right)} \\
& \cdot D_{*}^{\Sigma_{k_{0}}^{\dagger}}\left(w_{k_{0}}^{\dagger}\right)\left[\frac{\operatorname{vol}\left(\mathscr{C}_{i^{\prime}}^{\dagger}\right)}{\prod_{\lambda_{k_{0}}^{\dagger} \in \Sigma_{P_{k_{0}}^{\dagger}}^{0}-\Sigma_{P_{k_{0}}^{\dagger}}^{0}\left(w_{k_{0}}^{\dagger}\right)}\left(?, \lambda_{k_{0}}^{\dagger}\right)}\right. \\
& \left.\times\left\langle\mathbf{e}\left(\mathscr{C}_{k_{0}}: \mathbf{H}: ?\right), \operatorname{tr}_{\mathscr{C}_{k_{0}}}\left(\mathbf{T}_{k_{0} j_{0}}(\alpha: ?)\right)\right\rangle_{\mathscr{C}_{1}}\right]\left.\right|_{\left(1-w_{k_{0}}^{\dagger}\right) w_{k_{0} t_{0}}^{\dagger}\left(z \lambda_{i_{0}}+\Lambda_{i_{0}}\right)} d z\left|d \Lambda_{i_{0}}\right| .
\end{aligned}
$$

Technically, it will be advantageous at this point to choose a finite set $\Xi_{i_{0}}$ of $\xi_{i_{0}}$, each real of length one and in general position, connected by equivariance, i.e., with the property that

$$
\forall w_{j_{0} i_{0}}, \quad w_{j_{0} i_{0}} \boldsymbol{\Xi}_{i_{0}}=\Xi_{j_{0}} .
$$


We shall then write

$$
\int_{\mathfrak{C}\left(\Xi_{i_{0}}\right)} \phi\left(\Lambda_{i_{0}}\right) d \mathfrak{C}\left(\Lambda_{i_{0}}\right)
$$

in place of

$$
\begin{aligned}
\frac{1}{\sqrt{-1}} & \cdot \frac{1}{\#\left(\Xi_{i_{0}}\right)} \\
& \cdot \sum_{\xi_{i_{0}} \in \Xi_{i_{0}}} \int_{\operatorname{Re}\left(\Lambda_{i_{0}}\right)=0 \& \Lambda_{i_{0}} \perp \xi_{i_{0}}} \int_{\mathfrak{C}\left(\Lambda_{i_{0}}\right)} \phi\left(z \xi_{i_{0}}+\Lambda_{i_{0}}\right) d z\left|d \Lambda_{i_{0}}\right|
\end{aligned}
$$

This done, consider anew

$$
\sum_{k_{0}} \sum_{w_{k_{0} \iota_{0}}^{\dagger}} \sum_{w_{k_{0}}^{\dagger}} \int_{\mathfrak{C}\left(\Xi_{t_{0}}\right)}(-1)^{\#\left(\Sigma_{P_{k_{0}}^{\dagger}}^{0}\left(w_{k_{0}}^{\dagger}\right)\right)} \cdot D_{*}^{\Sigma_{p_{k_{0}}^{\dagger}}^{0}\left(w_{k_{0}}^{\dagger}\right)}[\cdots]_{\left(1-w_{k_{0}}^{\dagger}\right)} w_{k_{0^{\prime} 0}}^{\dagger} \Lambda_{i_{0}} d \mathfrak{C}\left(\Lambda_{i_{0}}\right)
$$

It will be convenient to make the change of variable

$$
\Lambda_{k_{0}}=w_{k_{0} i_{0}}^{\dagger} \Lambda_{i_{0}} \text {. }
$$

Per a pair $\left(k_{0}, w_{k_{0}}^{\dagger}\right)$, if $w_{k_{0} i_{0}}^{\dagger}$ runs over $W\left(A_{k_{0}}^{\dagger}, A_{i_{0}}^{\dagger}\right)$, then $w_{k_{0} j_{0}}^{-\dagger}$ runs over $W\left(A_{j_{0}}^{\dagger \prime}, A_{k_{0}}^{\dagger \prime}\right)$ in a

$$
\frac{\#\left(W\left(A_{k_{0}}^{\dagger}\right)\right)}{\#\left(W\left(A_{k_{0}}^{\dagger \prime}\right)\right)} \text {-to-one }
$$

fashion. Indeed, every $W\left(A_{k_{0}}^{\dagger \prime}\right)$-coset in $W\left(A_{k_{0}}^{\dagger}, A_{i_{0}}^{\dagger}\right)$ hits every $w_{k_{0} j_{0}}^{\dagger \prime}$ exactly once. It therefore follows that

$$
\sum_{k_{0}} \sum_{w_{k_{0} i_{0}}^{\dagger}} \sum_{w_{k_{0}}^{\dagger}} \int_{\mathfrak{C}\left(\Xi_{t_{0}}\right)}(-1)^{\#\left(\Sigma_{P_{k_{0}}^{\dagger}}^{0}\left(w_{k_{0}}^{\dagger}\right)\right)} \cdot D_{*}^{\Sigma_{P_{k_{0}}^{\dagger}}^{0}\left(w_{k_{0}}^{\dagger}\right)}[\cdots]_{\left(1-w_{k_{0}}^{\dagger}\right) w_{k_{0^{\prime}}}^{\dagger} \Lambda_{t_{0}}} d \mathfrak{C}\left(\Lambda_{i_{0}}\right)
$$

is equal to

$$
\sum_{k_{0}} \sum_{w_{k_{0}}^{\dagger}} \frac{\#\left(W\left(A_{k_{0}}^{\dagger}\right)\right)}{\left.\#\left(A_{k_{0}}^{\dagger \prime}\right)\right)} \cdot(-1)^{\#\left(\Sigma_{p_{0}}^{\dagger},\left(w_{k_{0}}^{\dagger}\right)\right)}
$$

times

$$
\sum_{w_{j_{0} k_{0}}^{\dagger \prime}} \int_{\mathfrak{C}\left(\Xi_{k_{0}}\right)} D_{*}^{\sum_{p_{k_{0}}^{\dagger}}^{0}\left(w_{k_{0}}^{\dagger}\right)}[\cdots]_{\left(1-w_{k_{0}}^{\dagger}\right) \Lambda_{k_{0}}} d \mathfrak{C}\left(\Lambda_{k_{0}}\right)
$$

Here, the term inside the square brackets is the product of

$$
\operatorname{vol}\left(\mathscr{C}_{i^{\prime}}^{\dagger}\right) / \prod_{\substack{\lambda_{k_{0}}^{\dagger} \in \Sigma_{P_{k_{0}}^{\dagger}}^{0}-\Sigma_{P_{k_{0}}^{+}}^{0}\left(w_{k_{0}}^{\dagger}\right)\\}}\left(?, \lambda_{k_{0}}^{\dagger}\right)
$$


and

$$
\left\langle\mathbf{e}\left(\mathscr{C}_{k_{0}}: \mathbf{H}: ?\right), \mathbf{t r}_{\mathscr{C}_{k_{0}}}\left(\mathbf{T}_{k_{0} j_{0}}(\alpha: ?)\right)\right\rangle_{\mathscr{C}_{l}},
$$

but now $\mathbf{T}_{k_{0} j_{0}}(\alpha: ?)$ is the consecutive composition of

$$
\left\{\begin{array}{l}
\mathbf{c}\left(P_{j_{0}}\left|A_{j_{0}}: P_{k_{0}}\right| A_{k_{0}}: w_{j_{0} k_{0}}^{\dagger \prime} w_{k_{0}}^{\dagger}:-\bar{\Lambda}_{k_{0}}\right)^{*}, \\
\mathbf{c}\left(P_{j_{0}}\left|A_{j_{0}}: P_{k_{0}}\right| A_{k_{0}}: w_{j_{0} k_{0}}^{\dagger \prime}: \Lambda_{k_{0}}-?\right)
\end{array}\right.
$$

and

$$
\text { Ind }_{\mathscr{E}_{k_{0}}}^{G}\left(\left(\boldsymbol{\sigma}_{k_{0}}, \Lambda_{k_{0}}\right)\right)(\alpha)
$$

For the moment, we shall view the orbit type $\boldsymbol{\theta}_{0}=\left\{\boldsymbol{\theta}_{i_{0}}\right\}$ as frozen. With this in mind, consider the sum

$$
\sum_{\mathscr{E}_{10}: \mathscr{E}_{i_{0}} \prec \mathscr{C}_{1}} .
$$

If, for the sake of brevity, $\mathscr{C}_{0}^{\dagger}$ is the daggered association class relative to $\mathscr{C}_{i}$ and $\mathscr{C}_{i_{0}}$ (cf. $\left.\S 5\right)$, then

$$
\sum_{\mathscr{E}_{1_{0}}: \mathscr{E}_{i_{0}} \prec \mathscr{C}_{1}}=\sum_{\mathscr{C}_{0}^{\dagger}} \sum_{\mathscr{C}_{i_{0}} \in \mathscr{C}_{0}^{\dagger}}
$$

Fix a $\mathscr{C}_{0}^{\dagger}$. Attached to each $\mathscr{C}_{i_{0}} \in \mathscr{C}_{0}^{\dagger}$ is the integral

$$
\int_{\operatorname{Re}\left(\Lambda_{i_{0}}\right)=0} \mathbf{I}_{\alpha}\left(\mathscr{C}_{i}: \boldsymbol{\theta}_{i_{0}}, \Lambda_{i_{0}}: \mathbf{H}\right)\left|d \Lambda_{i_{0}}\right|
$$

the value of which does not depend on $\mathscr{C}_{i_{0}}$. The number of contributing $\mathscr{C}_{i_{0}}$, i.e., $\#\left(\mathscr{C}_{0}^{\dagger}\right)$, is

$$
\frac{*\left(\mathscr{C}_{i_{0}}^{\dagger}\right)}{\#\left(W\left(A_{i_{0}}^{\dagger}\right)\right)} \quad\left(=\frac{*\left(\mathscr{C}_{k_{0}}^{\dagger}\right)}{\#\left(W\left(A_{k_{0}}^{\dagger}\right)\right)}\right) .
$$

Accordingly,

$$
\begin{aligned}
& \sum_{\mathscr{E}_{i_{0}} \in \mathscr{C}_{0}^{\dagger}} \int_{\operatorname{Re}\left(\Lambda_{i_{0}}\right)=0} \mathbf{I}_{\alpha}\left(\mathscr{C}_{i}: \boldsymbol{\sigma}_{i_{0}}, \Lambda_{i_{0}}: \mathbf{H}\right)\left|d \Lambda_{i_{0}}\right| \\
& =\sum_{\mathscr{C}_{i_{0}} \in \mathscr{C}_{0}^{\dagger}} \int_{\operatorname{Re}\left(\Lambda_{i_{0}}\right)=0} \mathbf{0}_{\mathscr{C}_{t}}\left(\boldsymbol{\sigma}_{i_{0}}, \Lambda_{i_{0}}: \mathbf{H}\right)\left|d \Lambda_{i_{0}}\right| \\
& +(-1)^{l_{0}^{\dagger}} \cdot \sum_{k_{0}} \sum_{w_{k_{0}}^{\dagger}} \frac{*\left(\mathscr{C}_{k_{0}}^{\dagger}\right)}{\#\left(W\left(A_{k_{0}}^{\dagger \prime}\right)\right)} \cdot(-1)^{\#\left(\Sigma_{k_{0}}^{0}\left(w_{k_{0}}^{\dagger}\right)\right)} \\
& \times \sum_{w_{J_{0} k_{0}}^{\dagger+}} \int_{\mathfrak{C}\left(\Xi_{k_{0}}\right)} D_{*}^{\Sigma_{P_{k_{0}}^{\dagger}}^{0}\left(w_{k_{0}}^{\dagger}\right)}[\cdots]_{\left(1-w_{k_{0}}^{\dagger}\right) \Lambda_{k_{0}}} d \mathfrak{C}\left(\Lambda_{k_{0}}\right)
\end{aligned}
$$


A given pair $\left(k_{0}, w_{k_{0}}^{\dagger}\right)$ singles out a choice of $j_{0}$ (cf. supra). If this particular choice is changed but the others are kept fixed, then what has been said above is still true. In other words, our formula for

$$
\sum_{\mathscr{E}_{i_{0}} \in \mathscr{C}_{0}^{\dagger}} \int_{\operatorname{Re}\left(\Lambda_{i_{0}}\right)=0} \mathbf{I}_{\alpha}\left(\mathscr{C}_{i}: \boldsymbol{\sigma}_{i_{0}}, \Lambda_{i_{0}}: \mathbf{H}\right)\left|d \Lambda_{i_{0}}\right|
$$

must be independent of $j_{0}$. Because the number of $j_{0}$ at issue is

$$
\frac{*\left(\mathscr{C}_{k_{0}}^{\dagger \prime}\right)}{\#\left(W\left(A_{k_{0}}^{\dagger \prime}\right)\right)}
$$

upon averaging over $j_{0}$,

$$
\begin{aligned}
(-1)^{l_{0}^{\dagger}} & \cdot \sum_{k_{0}} \sum_{w_{k_{0}}^{\dagger}} \frac{*\left(\mathscr{C}_{k_{0}}^{\dagger}\right)}{\#\left(W\left(A_{k_{0}}^{\dagger \prime}\right)\right)} \cdot(-1)^{\#\left(\Sigma_{P_{k_{0}}^{\dagger}}^{0}\left(w_{k_{0}}^{\dagger}\right)\right)} \\
& \times \sum_{w_{j_{0} k_{0}}^{\dagger}} \int_{\mathfrak{C}\left(\Xi_{k_{0}}\right)} D_{*}^{\Sigma_{P_{k_{0}}^{\dagger}}^{0}\left(w_{k_{0}}^{\dagger}\right)}[\cdots]_{\left(1-w_{k_{0}}^{\dagger}\right) \Lambda_{k_{0}}} d \mathfrak{C}\left(\Lambda_{k_{0}}\right)
\end{aligned}
$$

is still

$$
\begin{aligned}
(-1)^{l_{0}^{\dagger}} & \cdot \sum_{k_{0}} \sum_{w_{k_{0}}^{\dagger}} \frac{*\left(\mathscr{C}_{k_{0}}^{\dagger}\right)}{*\left(\mathscr{C}_{k_{0}}^{\dagger \prime}\right)} \cdot(-1)^{\#\left(\Sigma_{p_{k_{0}}^{\dagger}}^{0}\left(w_{k_{0}}^{\dagger}\right)\right)} \\
& \times \sum_{j_{0}} \sum_{w_{j_{0} k_{0}}^{\dagger \prime}} \int_{\mathfrak{C}\left(\Xi_{k_{0}}\right)} D_{*}^{\sum_{P_{k_{0}}^{\dagger}}^{0}\left(w_{k_{0}}^{\dagger}\right)}[\cdots]_{\left(1-w_{k_{0}}^{\dagger}\right) \Lambda_{k_{0}}} d \mathfrak{C}\left(\Lambda_{k_{0}}\right)
\end{aligned}
$$

Implicitly, the contour of integration depends on the orbit type $\theta_{0}=$ $\left\{\boldsymbol{\sigma}_{i_{0}}\right\}$, although this is not reflected in the notation (and causes no 
problems). Summing up:

Proposition 9.4. Let $\alpha$ be a $K$-central, $K$-finite element of $C_{c}^{\infty}(G)-$ then

$$
\begin{aligned}
& \int_{\operatorname{Re}\left(\Lambda_{t}\right)=0} \sum_{\mathscr{C}_{l_{0}} \in \mathscr{E}_{0}^{\dagger}} \sum_{\boldsymbol{\theta}_{l_{0}}} \int_{\operatorname{Re}\left(\Lambda_{t_{0}}^{\dagger}\right)=0} \mathbf{I}_{\alpha}\left(\mathscr{C}_{i}: \boldsymbol{\theta}_{i_{0}}, \Lambda_{i}+\Lambda_{l_{0}}^{\dagger}: \mathbf{H}\right)\left|d \Lambda_{i_{0}}^{\dagger}\right|\left|d \Lambda_{l}\right| \\
& =\sum_{\mathscr{C}_{i_{0}} \in \mathscr{C}_{0}^{\dagger}} \sum_{\boldsymbol{O}_{i_{0}}} \int_{\operatorname{Re}\left(\Lambda_{t_{0}}\right)=0} \mathbf{0}_{\mathscr{L}_{l}}\left(\boldsymbol{\sigma}_{i_{0}}, \Lambda_{i_{0}}: \mathbf{H}\right)\left|d \Lambda_{i_{0}}\right| \\
& +(-1)^{l_{0}^{\dagger}} \cdot \sum_{k_{0}} \sum_{\boldsymbol{O}_{k_{0}}} \sum_{w_{k_{0}}^{\dagger}} \frac{*\left(\mathscr{C}_{k_{0}}^{\dagger}\right)}{*\left(\mathscr{C}_{k_{0}}^{\dagger \prime}\right)} \cdot(-1)^{\#\left(\Sigma_{p_{0}}^{0}\left(w_{k_{0}}^{\dagger}\right)\right)} \\
& \times \sum_{j_{0}} \sum_{w_{j_{0} k_{0}}^{\dagger \prime}} \int_{\mathfrak{C}\left(\Xi_{k_{0}}\right)} D_{*}^{\Sigma_{P_{k_{0}}^{+\prime}}^{0}\left(w_{k_{0}}^{\dagger}\right)}[\cdots]_{\left(1-w_{k_{0}}^{\dagger}\right) \Lambda_{k_{0}}} d \mathfrak{C}\left(\Lambda_{k_{0}}\right),
\end{aligned}
$$

$[\cdots]$ being the product of

and

$$
\operatorname{vol}\left(\mathscr{C}_{i^{\prime}}^{\dagger}\right) / \prod_{\substack{\lambda_{k_{0}}^{\dagger} \in \Sigma_{P_{k_{0}}^{\dagger}}^{0}-\Sigma_{P_{k_{0}}^{+}}\left(w_{k_{0}}^{\dagger}\right)\\}}\left(?, \lambda_{k_{0}}^{\dagger}\right)
$$

$$
\left\langle\mathbf{e}\left(\mathscr{C}_{k_{0}}: \mathbf{H}: ?\right), \mathbf{t r}_{\mathscr{C}_{k_{0}}}\left(\mathbf{T}_{k_{0} j_{0}}(\alpha: ?)\right)\right\rangle_{\mathscr{C}_{i}} \text {. }
$$

All the integral-series are absolutely integrable-summable.

At this juncture, it may be helpful to remind ourselves that

$$
\operatorname{tr}_{\mathscr{E}_{l}}\left(\mathbf{Q}^{\mathbf{I}_{\mathscr{E}_{l}}(\mathbf{H})} \circ \mathbf{L}_{\mathscr{E}_{l} / \Gamma_{\mathscr{E}_{l}}}^{\text {con }}\left(\alpha^{\mathscr{E}_{I}}\right) \circ \mathbf{Q}^{\mathbf{I}_{\mathscr{E}_{l}}(\mathbf{H})}\right)
$$

is

$$
\frac{1}{(2 \pi)^{l_{l}}} \cdot \int_{\operatorname{Re}\left(\Lambda_{t}\right)=0} \cdots\left|d \Lambda_{i}\right|
$$

... being

$$
\begin{aligned}
\sum_{\mathscr{C}_{0}^{\dagger}} & \sum_{\mathscr{E}_{i_{0}} \in \mathscr{C}_{0}^{\dagger}} \sum_{\boldsymbol{\theta}_{i_{0}}} \frac{1}{(2 \pi)^{l_{0}^{\dagger}}} \cdot \frac{1}{*\left(\mathscr{C}_{i_{0}}^{\dagger}\right)} \\
& \times \int_{\operatorname{Re}\left(\Lambda_{i_{0}}^{\dagger}\right)=0} \mathbf{I}_{\alpha}\left(\mathscr{C}_{i}: \boldsymbol{\theta}_{i_{0}}, \Lambda_{i}+\Lambda_{i_{0}}: \mathbf{H}\right)\left|d \Lambda_{i_{0}}^{\dagger}\right|
\end{aligned}
$$

So, the preceding proposition may be regarded as giving a semiexplicit expression for

$$
\operatorname{tr}_{\mathscr{E}_{1}}\left(\mathbf{Q}^{\mathbf{I}_{\mathscr{E}_{1}}(\mathbf{H})} \circ \mathbf{L}_{\mathscr{C}_{1} / \Gamma_{\mathscr{E}_{1}}}^{\text {con }}\left(\alpha^{\mathscr{E}_{1}}\right) \circ \mathbf{Q}^{\mathbf{I}_{\mathscr{I}_{1}}(\mathbf{H})}\right)
$$


Eventually, it will be shown that the

$$
\mathbf{0}_{\mathscr{C}_{l}}\left(\boldsymbol{\sigma}_{i_{0}}, \Lambda_{i_{0}}: \mathbf{H}\right)
$$

terms can be ignored. As for the

$$
D_{*}^{\sum_{p_{0}^{\dagger}}^{0}\left(w_{k_{0}}^{\dagger}\right)}[\cdots]_{\left(1-w_{k_{0}}^{\dagger}\right) \Lambda_{k_{0}}}
$$

terms, with patience it is possible to make them a good deal more transparent.

To this end, fix $j_{0}, \Lambda_{j_{0}}, w_{j_{0}}^{\dagger}$ and introduce a parameter $\mathbf{H}_{\mathrm{OO}} \in \mathfrak{a}$. Consider

$$
\sum_{k_{0}} \sum_{w_{j_{0} k_{0}}^{\dagger+}} D_{*}^{\Sigma_{P_{k_{0}}^{\dagger}}^{0}\left(w_{k_{0}}^{\dagger}\right)}[\cdots]_{\left(1-w_{k_{0}}^{\dagger}\right) \Lambda_{k_{0}}} .
$$

According to our definition (cf. $§ 5)$, the

$$
\mathbf{e}\left(\mathscr{C}_{k_{0}}: \mathbf{H}: ?\right)
$$

appearing in the expression

$$
\left\langle\mathbf{e}\left(\mathscr{C}_{k_{0}}: \mathbf{H}: ?\right), \operatorname{tr}_{\mathscr{C}_{k_{0}}}\left(\mathbf{T}_{k_{0} j_{0}}(\alpha: ?)\right)\right\rangle_{\mathscr{C}_{1}}
$$

is a certain element of $\mathbf{C}^{r_{k_{0}}}$. But we can also view

$$
\mathbf{e}\left(\mathscr{C}_{k_{0}}: \mathbf{H}: ?\right)
$$

as an operator on $\mathscr{E}\left(\delta, \boldsymbol{\theta}_{k_{0}}\right)$ by formal multiplication of row and column vectors. With this understanding, we have

$$
\left\langle\mathbf{e}\left(\mathscr{C}_{k_{0}}: \mathbf{H}: ?\right), \operatorname{tr}_{\mathscr{C}_{k_{0}}}\left(\mathbf{T}_{k_{0} j_{0}}(\alpha: ?)\right)\right\rangle_{\mathscr{C}_{1}}=\operatorname{tr}_{\mathscr{C}_{1}}(A \circ B),
$$

where

or still

$$
\left\{\begin{aligned}
A= & \mathbf{e}\left(\mathscr{C}_{k_{0}}: \mathbf{H}-\mathbf{H}_{\mathrm{OO}}: ?\right) \\
& \cdot \mathbf{c}\left(P_{j_{0}}\left|A_{j_{0}}: P_{k_{0}}\right| A_{k_{0}}: w_{j_{0} k_{0}}^{\dagger \prime} w_{k_{0}}^{\dagger}:-\bar{\Lambda}_{k_{0}}\right)^{*}, \\
B= & \mathbf{c}\left(P_{j_{0}}\left|A_{j_{0}}: P_{k_{0}}\right| A_{k_{0}}: w_{j_{0} k_{0}}^{\dagger}: \Lambda_{k_{0}}-?\right) \\
& \cdot \mathbf{e}\left(\mathscr{C}_{k_{0}}: \mathbf{H}_{\mathrm{OO}}: ?\right) \cdot \mathbf{I n d}_{\mathscr{C}_{k_{0}}}^{G}\left(\left(\boldsymbol{\sigma}_{k_{0}}, \Lambda_{k_{0}}\right)\right)(\alpha)
\end{aligned}\right.
$$

where

$$
\operatorname{tr}_{\mathscr{C}_{l}}\left(\mathbf{c}_{j_{0} k_{0}} \circ A \circ B \circ \mathbf{c}_{j_{0} k_{0}}^{-1}\right)
$$

$$
\left\{\begin{aligned}
A= & \mathbf{e}\left(\mathscr{C}_{k_{0}}: \mathbf{H}-\mathbf{H}_{\mathrm{OO}}: ?\right) \\
& \cdot \mathbf{c}\left(P_{j_{0}}\left|A_{j_{0}}: P_{k_{0}}\right| A_{k_{0}}: w_{j_{0} k_{0}}^{\dagger \prime} w_{k_{0}}^{\dagger}:-w_{j_{0} k_{0}}^{-\dagger} \bar{\Lambda}_{j_{0}}\right)^{*}, \\
B= & \mathbf{c}\left(P_{j_{0}}\left|A_{j_{0}}: P_{k_{0}}\right| A_{k_{0}}: w_{j_{0} k_{0}}^{\dagger \prime}: w_{j_{0} k_{0}}^{-\dagger \prime} \Lambda_{j_{0}}-?\right) \\
& \cdot \mathbf{e}\left(\mathscr{C}_{k_{0}}: \mathbf{H}_{\mathrm{OO}}: ?\right) \cdot \mathbf{I n d}_{\mathscr{6}_{k_{0}}}^{G}\left(\left(\boldsymbol{O}_{k_{0}}, w_{j_{0} k_{0}}^{-\dagger} \Lambda_{j_{0}}\right)\right)(\alpha)
\end{aligned}\right.
$$


and

$$
\mathbf{c}_{j_{0} k_{0}}=\mathbf{c}\left(P_{j_{0}}\left|A_{j_{0}}: P_{k_{0}}\right| A_{k_{0}}: w_{j_{0} k_{0}}^{\dagger \prime}: w_{j_{0} k_{0}}^{-\dagger \prime} \Lambda_{j_{0}}\right) .
$$

The point here is that

$$
\mathbf{c}_{j_{0} k_{0}} \circ A \circ B \circ \mathbf{c}_{j_{0} k_{0}}^{-1}
$$

is an endomorphism of $\mathscr{E}\left(\delta, \boldsymbol{\sigma}_{j_{0}}\right)$.

To simplify the notation, set

$$
\Lambda_{k_{0}}=w_{j_{0} k_{0}}^{-\dagger \prime} \Lambda_{j_{0}}
$$

In addition, put

$$
\Lambda_{k_{0}}^{\dagger \prime}=w_{j_{0} k_{0}}^{-\dagger \prime} \Lambda_{j_{0}}^{\dagger \prime}
$$

and write

$$
\Lambda=\left(1-w_{k_{0}}^{\dagger}\right) \Lambda_{k_{0}}+\Lambda_{k_{0}}^{\dagger \prime}
$$

At $\Lambda$, then,

$$
\left\langle\mathbf{e}\left(\mathscr{C}_{k_{0}}: \mathbf{H}: ?\right), \operatorname{tr}_{\mathscr{C}_{k_{0}}}\left(\mathbf{T}_{k_{0} j_{0}}(\alpha: ?)\right)\right\rangle_{\mathscr{C}_{i}}
$$

is equal to

$$
\operatorname{tr}_{\mathscr{C}_{i}}\left(\mathbf{c}_{j_{0} k_{0}} \circ A(\Lambda) \circ B(\Lambda) \circ \mathbf{c}_{j_{0} k_{0}}^{-1}\right)
$$

where

$$
\left\{\begin{aligned}
A(\Lambda)= & \mathbf{e}\left(\mathscr{C}_{k_{0}}: \mathbf{H}-\mathbf{H}_{\mathrm{OO}}: \Lambda\right) \\
& \cdot \mathbf{c}\left(P_{j_{0}}\left|A_{j_{0}}: P_{k_{0}}\right| A_{k_{0}}: w_{j_{0} k_{0}}^{\dagger \prime} w_{k_{0}}^{\dagger}:-\bar{\Lambda}_{k_{0}}\right)^{*}, \\
B(\Lambda)= & \mathbf{c}\left(P_{j_{0}}\left|A_{j_{0}}: P_{k_{0}}\right| A_{k_{0}}: w_{j_{0} k_{0}}^{\dagger \prime}: \Lambda_{k_{0}}-\Lambda\right) \\
& \cdot \mathbf{e}\left(\mathscr{C}_{k_{0}}: \mathbf{H}_{\mathrm{OO}}: \Lambda\right) \cdot \mathbf{I n d}_{\mathscr{C}_{k_{0}}}^{G}\left(\left(\boldsymbol{O}_{k_{0}}, \Lambda_{k_{0}}\right)\right)(\alpha),
\end{aligned}\right.
$$

and

$$
\mathbf{c}_{j_{0} k_{0}}=\mathbf{c}\left(P_{j_{0}}\left|A_{j_{0}}: P_{k_{0}}\right| A_{k_{0}}: w_{j_{0} k_{0}}^{\dagger \prime}: \Lambda_{k_{0}}\right) .
$$

Observe that $\Lambda_{j_{0}}^{\dagger \prime}$ is now the variable. Letting it equal zero is tantamount to evaluation at $\left(1-w_{k_{0}}^{\dagger}\right) \Lambda_{k_{0}}$. That being, our objective will be to interpret

$$
\sum_{k_{0}} \sum_{w_{j_{0} k_{0}}^{\dagger \prime}} D_{*}^{\sum_{P_{k_{0}}^{\dagger \prime}}^{0}\left(w_{k_{0}}^{\dagger}\right)}[\cdots]_{\left(1-w_{k_{0}}^{\dagger}\right) \Lambda_{k_{0}}}
$$

by a suitable application of Proposition 4.5. This is in fact the reason for our temporary shift in emphasis:

$$
\left\{\begin{array}{l}
j_{0} \text { is the "base-point" for Detroit families, } \\
k_{0} \text { is the "base-point" for integration. }
\end{array}\right.
$$


Qua functions of $\Lambda_{j_{0}}^{\dagger \prime}$, we have

$$
\left\{\begin{array}{l}
(A) \mathbf{c}_{j_{0} k_{0}} \circ A(\Lambda), \\
(B) B(\Lambda) \circ \mathbf{c}_{j_{0} k_{0}}^{-1},
\end{array} \quad(C) C(\Lambda)\right.
$$

$C(\Lambda)$ the product

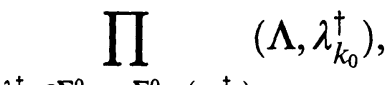

$$
\begin{aligned}
& \lambda_{k_{0}}^{\dagger} \in \Sigma_{P_{k_{0}}^{\dagger}}^{0}-\Sigma_{P_{k_{0}}^{\dagger}}^{0}\left(w_{k_{0}}^{\dagger}\right)
\end{aligned}
$$

and we claim:

Lemma 9.5. The functions in $A, B$, and $C$ constitute Detroit families on $\sqrt{-1} \grave{\mathfrak{a}}_{j_{0}}^{\dagger \prime}$.

[Note: The "chamber index" is the pair $\left(k_{0}, w_{j_{0} k_{0}}^{\dagger \prime}\right)$. ]

The verification of this lemma depends on nothing more than the functional equations, thus runs along familiar lines and so the details can be omitted.

With this preparation, to apply Proposition 4.5 (in its operatortheoretic guise), we must specify $\phi$ and $\psi$, as well as the $f\left(W, \mathscr{C}_{W}\right)$. The choice for $\phi$ and $\psi$ is easy enough, namely let

$$
\left\{\begin{array}{l}
\phi=A \times \operatorname{vol}\left(\mathscr{C}_{i^{\prime}}^{\dagger}\right) C^{-1}, \\
\psi=B .
\end{array}\right.
$$

Turning to the $f\left(W, \mathscr{C}_{W}\right)$, fixing ( $\left.W, \mathscr{C}_{W}\right)$ amounts to picking a daggered association class based at $\left(k_{0}, w_{j_{0} k_{0}}^{\dagger \prime}\right)$, producing data

$$
\left(l_{0}, w_{k_{0} l_{0}}^{\dagger \prime}\right), w_{k_{0} l_{0}}^{\dagger \prime} \in W\left(A_{k_{0}}(F), \ldots\right) \quad\left(F \subset \Sigma_{P_{k_{0}}^{\dagger \prime}}^{0}\left(w_{k_{0}}^{\dagger}\right)\right) .
$$

With this in mind, let

$$
\begin{aligned}
f\left(W, \mathscr{C}_{W}\right)= & \frac{1}{C_{k_{0}}(F)} \cdot \sum_{l_{0}} \sum_{w_{k_{0} l_{0}}^{\dagger \prime}} \mathbf{c}\left(P_{j_{0}}\left|A_{j_{0}}: P_{l_{0}}\right| A_{l_{0}}: w_{j_{0} k_{0}}^{\dagger \prime} w_{k_{0} l_{0}}^{\dagger \prime}:\right. \\
& \times \mathbf{c}\left(P_{j_{0}}\left|A_{j_{0}}: P_{l_{0}}\right| A_{l_{0}}: w_{j_{0} k_{0}}^{\dagger \prime} w_{k_{0} l_{0}}^{\dagger \prime}: \quad\left(w_{j_{0} k_{0}}^{\dagger \prime} w_{k_{0} l_{0}}^{\dagger \prime}\right)^{-1} w_{j_{0}}^{\dagger} \Lambda_{j_{0}}\right) \\
& \left.\left(w_{j_{0} k_{0}}^{\dagger \prime} w_{k_{0} l_{0}}^{\dagger \prime}\right)^{-1}\left(w_{j_{0}}^{\dagger} \Lambda_{j_{0}}-\Lambda_{j_{0}}^{\dagger \prime}\right)\right)^{-1} .
\end{aligned}
$$

Here, $C_{k_{0}}(F)$ is the number of chambers in $\mathfrak{a}_{k_{0}}(F)$. In view of the definitions,

$$
\Lambda_{k_{0}}-\Lambda=w_{k_{0}}^{\dagger} \Lambda_{k_{0}}-\Lambda_{k_{0}}^{\dagger}
$$


Also, it is clear that

$$
f\left(W, \mathscr{C}_{W}\right)(0)=\text { ID } .
$$

Finally, the $\Lambda$-dependent terms in

$$
f\left(W, \mathscr{C}_{W}\right) \cdot \boldsymbol{\psi}\left(\mathscr{C}_{W}\right)
$$

reduce, via the functional equations, to

$$
\mathbf{c}\left(P_{l_{0}}\left|A_{l_{0}}: P_{k_{0}}\right| A_{k_{0}}: w_{k_{0} l_{0}}^{-\dagger \prime}: \Lambda_{k_{0}}-\Lambda\right) \cdot \mathbf{e}\left(\mathscr{C}_{k_{0}}: \mathbf{H}_{\mathrm{OO}}: \Lambda\right) .
$$

Returning to

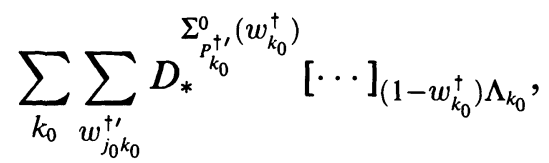

bring $\operatorname{tr}_{\mathscr{C}_{i}}$ to the outside-then what is left inside is precisely

$$
( \pm) \mathbf{U}_{\phi \cdot \boldsymbol{\psi}}(0)
$$

computable by Proposition 4.5 as the sum

$$
\sum_{k_{0}} \sum_{w_{J_{0} k_{0}}^{\dagger \prime}} \sum_{F} \frac{1}{C_{k_{0}}(F)} \cdot \sum_{l_{0}} \sum_{w_{k_{0} l_{0}}^{\dagger \prime}}
$$

of the

$$
\begin{aligned}
& \mathbf{c}\left(P_{j_{0}}\left|A_{j_{0}}: P_{k_{0}}\right| A_{k_{0}}: w_{j_{0} k_{0}}^{\dagger \prime}: \Lambda_{k_{0}}\right) \\
& \times \operatorname{vol}\left(\mathscr{C}_{i^{\prime}}^{\dagger}\right) \cdot D_{F}^{\Sigma_{P_{k_{0}}^{\dagger}}^{0}\left(w_{k_{0}}^{\dagger}\right)}\left[\frac{\mathbf{e}\left(\mathscr{C}_{k_{0}}: \mathbf{H}-\mathbf{H}_{\mathrm{OO}}: ?\right)}{\prod_{\lambda_{k_{0}}^{\dagger} \in \Sigma_{P_{k_{0}}^{\dagger}}^{0}-\Sigma_{P_{k_{0}}^{\dagger}}^{0}\left(w_{k_{0}}^{\dagger}\right)}\left(?, \lambda_{k_{0}}^{\dagger}\right)}\right]_{\left(1-w_{k_{0}}^{\dagger}\right) \Lambda_{k_{0}}} \\
& \times \mathbf{c}\left(P_{l_{0}}\left|A_{l_{0}}: P_{k_{0}}\right| A_{k_{0}}: w_{k_{0} l_{0}}^{-\dagger \prime} w_{k_{0}}^{\dagger}:-\bar{\Lambda}_{k_{0}}\right)^{*} \\
& \times D_{*}^{F}\left[\mathbf{c}\left(P_{l_{0}}\left|A_{l_{0}}: P_{k_{0}}\right| A_{k_{0}}: w_{k_{0} l_{0}}^{-\dagger \prime}: \Lambda_{k_{0}}-?\right)\right. \\
& \left.\cdot \mathbf{e}\left(\mathscr{C}_{k_{0}}: \mathbf{H}_{\mathrm{OO}}: ?\right)\right]_{\left(1-w_{k_{0}}^{\dagger}\right) \Lambda_{k_{0}}} \\
& \times \operatorname{Ind}_{\mathscr{\delta}_{k_{0}}}^{G}\left(\left(\boldsymbol{\sigma}_{k_{0}}, \Lambda_{k_{0}}\right)\right)(\alpha) \\
& \times \mathbf{c}\left(P_{j_{0}}\left|A_{j_{0}}: P_{k_{0}}\right| A_{k_{0}}: w_{j_{0} k_{0}}^{\dagger \prime}: \Lambda_{k_{0}}\right)^{-1} \text {. }
\end{aligned}
$$

Take now $\mathbf{t r}_{\mathscr{C}_{1}}$-then the c-functions at the beginning and the end disappear and we find that

$$
\sum_{k_{0}} \sum_{w_{\jmath_{0} k_{0}}^{\dagger \prime}} D_{*}^{\Sigma_{P_{k_{0}}^{+\prime}}^{0}\left(w_{k_{0}}^{\dagger}\right)}[\cdots]_{\left(1-w_{k_{0}}^{\dagger}\right) \Lambda_{k_{0}}}
$$


is expressible as the sum

$$
\sum_{k_{0}} \sum_{w_{j_{0} k_{0}}^{\dagger \prime}} \sum_{F} \frac{1}{C_{k_{0}}(F)} \cdot \sum_{l_{0}} \sum_{\substack{w_{k_{0} l_{0}}^{\dagger \prime} \\ \text { ing }}}
$$

of $\operatorname{vol}\left(\mathscr{C}_{i^{\prime}}^{\dagger}\right)$ times

$$
\left\langle D_{F}^{\Sigma_{p_{0}}^{\dagger}\left(w_{k_{0}}^{\dagger}\right)}\left[\frac{\mathbf{e}\left(\mathscr{C}_{k_{0}}: \mathbf{H}-\mathbf{H}_{\mathrm{OO}}: ?\right)}{\prod_{\lambda_{k_{0}}^{\dagger} \in \Sigma_{P_{k_{0}}^{\dagger}}^{0}-\Sigma_{P_{k_{0}}^{\prime \prime}}^{0}\left(w_{k_{0}}^{\dagger}\right)}\left(?, \lambda_{k_{0}}^{\dagger}\right)}\right], D_{*}^{F}\left[\mathbf{t r}_{\mathscr{C}_{k_{0}}}\left(\mathbf{T}_{k_{0} l_{0}}\left(\alpha: \mathbf{H}_{\mathrm{OO}}: ?\right)\right)\right]\right\rangle_{\mathscr{C}_{l_{1}}},
$$

both derivatives being evaluated at $\left(1-w_{k_{0}}^{\dagger}\right) \Lambda_{k_{0}}$.

To place this conclusion in perspective, a series of simple combinatorial observations will be needed. Thus, looking at

$$
\sum_{k_{0}} \sum_{w_{k_{0}}^{\dagger}}( \pm) \frac{*\left(\mathscr{C}_{k_{0}}^{\dagger}\right)}{*\left(\mathscr{C}_{k_{0}}^{\dagger \dagger}\right)} \cdot \sum_{j_{0}} \sum_{w_{j_{0} k_{0}}^{\dagger+}} \int_{\mathfrak{C}\left(\Xi_{k_{0}}\right)} D_{*}^{D_{p_{k_{0}}^{\dagger}}^{\Sigma_{1}^{\dagger}}\left(w_{k_{0}}^{\dagger}\right)}[\cdots]
$$

is the same as looking at

$$
\sum_{j_{0}} \sum_{w_{J_{0}}^{\dagger}}( \pm) \frac{*\left(\mathscr{C}_{j_{0}}^{\dagger}\right)}{*\left(\mathscr{C}_{j_{0}}^{\dagger}\right)} \cdot \int_{\mathfrak{C}\left(\Xi_{J_{0}}\right)} \sum_{k_{0}} \sum_{w_{J_{0} k_{0}}^{\dagger \prime}} D_{*}^{\Sigma_{p_{k_{0}}^{\dagger \prime}}^{0}\left(w_{k_{0}}^{\dagger}\right)}[\cdots]
$$

or still

$$
\begin{aligned}
\sum_{j_{0}} \sum_{w_{j_{0}}^{\dagger}}( \pm) \frac{*\left(\mathscr{C}_{j_{0}}^{\dagger}\right)}{*\left(\mathscr{C}_{j_{0}}^{\dagger \prime}\right)} \cdot \int_{\mathfrak{C}\left(\Xi_{j_{0}}\right)} \sum_{k_{0}} \sum_{w_{j_{0} k_{0}}^{\dagger \prime}} \sum_{F} \frac{1}{C_{k_{0}}(F)} \\
\cdot \sum_{l_{0}} \sum_{w_{k_{0_{0}} l_{0}}^{\dagger \prime}} \operatorname{vol}\left(\mathscr{C}_{i^{\prime}}^{\dagger}\right) \cdot\left\langle D_{F}^{\Sigma_{p_{k_{0}}^{\dagger}}^{\dagger}\left(w_{k_{0}}^{\dagger}\right)}[\cdots], D_{*}^{F}[\cdots]\right\rangle_{\mathscr{C}_{l}}
\end{aligned}
$$

or still

$$
\begin{aligned}
\sum_{k_{0}} \sum_{w_{k_{0}}^{\dagger}}( \pm) \frac{*\left(\mathscr{C}_{k_{0}}^{\dagger}\right)}{*\left(\mathscr{C}_{k_{0}}^{\dagger \dagger}\right)} \cdot \sum_{j_{0}} \sum_{w_{j_{0} k_{0}}^{\dagger+}} \int_{\mathfrak{C}\left(\Xi_{k_{0}}\right)} \sum_{F} \frac{1}{C_{k_{0}}(F)} \\
\cdot \sum_{l_{0}} \sum_{w_{k_{0} l_{0}}^{\dagger \prime}} \operatorname{vol}\left(\mathscr{C}_{i^{\prime}}^{\dagger}\right) \cdot\left\langle D_{F}^{\Sigma_{k_{0}}^{\dagger}\left(w_{k_{0}}^{\dagger}\right)}[\cdots], D_{*}^{F}[\cdots]\right\rangle_{\mathscr{C}_{i}} .
\end{aligned}
$$


But every $\left(j_{0}, w_{j_{0} k_{0}}^{\dagger \prime}\right)$-term is the same and there are $*\left(\mathscr{C}_{k_{0}}^{\dagger \prime}\right)$ of them, giving at last

$$
\begin{aligned}
& \sum_{k_{0}} \sum_{w_{k_{0}}^{\dagger}} *\left(\mathscr{C}_{k_{0}}^{\dagger}\right)( \pm) \\
& \quad \times \int_{\mathfrak{C}_{\left(\Xi_{k_{0}}\right)}} \sum_{F} \frac{1}{C_{k_{0}}(F)} \cdot \sum_{l_{0}} \sum_{w_{k_{0} l_{0}}^{\dagger \prime}} \operatorname{vol}\left(\mathscr{C}_{i^{\prime}}^{\dagger}\right) \\
& \quad \cdot\left\langle D_{F}^{\Sigma_{P_{k_{0}}^{\dagger}}^{0}\left(w_{k_{0}}^{\dagger}\right)}[\cdots], D_{*}^{F}[\cdots]\right\rangle_{\mathscr{C}_{l}} \cdot
\end{aligned}
$$

Hence:

Proposition 9.6. Let $\alpha$ be a $K$-central, $K$-finite element of $C_{c}^{\infty}(G)-$ then

$$
\begin{aligned}
& \int_{\operatorname{Re}\left(\Lambda_{i}\right)=0} \sum_{\mathscr{E}_{i_{0}} \in \mathscr{E}_{0}^{\dagger}} \sum_{\boldsymbol{\theta}_{i_{0}}} \int_{\operatorname{Re}\left(\Lambda_{i_{0}}^{\dagger}\right)=0} \mathbf{I}_{\alpha}\left(\mathscr{C}_{i}: \boldsymbol{\theta}_{i_{0}}, \Lambda_{i}+\Lambda_{i_{0}}^{\dagger}: \mathbf{H}\right)\left|d \Lambda_{i_{0}}^{\dagger}\right|\left|d \Lambda_{i}\right| \\
& =\sum_{\mathscr{E}_{i_{0}} \in \mathscr{E}_{0}^{\dagger}} \sum_{\boldsymbol{\sigma}_{i_{0}}} \int_{\operatorname{Re}\left(\Lambda_{i_{0}}\right)=0} \mathbf{O}_{\mathscr{E}_{i}}\left(\boldsymbol{\sigma}_{i_{0}}, \Lambda_{i_{0}}: \mathbf{H}\right)\left|d \Lambda_{i_{0}}\right| \\
& +(-1)^{l_{0}^{\dagger}} \cdot \sum_{k_{0}} \sum_{\mathscr{O}_{k_{0}}} \sum_{w_{k_{0}}^{\dagger}} *\left(\mathscr{C}_{k_{0}}^{\dagger}\right) \cdot(-1)^{\#\left(\Sigma_{P_{k_{0}}^{\dagger}}^{0}\left(w_{k_{0}}^{\dagger}\right)\right)} \\
& \times \sum_{F} \frac{1}{C_{k_{0}}(F)} \cdot \sum_{l_{0}} \sum_{w_{k_{0} l_{0}}^{\dagger \prime}} \operatorname{vol}\left(\mathscr{C}_{i^{\prime}}^{\dagger}\right) \\
& \times \int_{\mathfrak{C}\left(\Xi_{k_{0}}\right)}\left\langle D_{F}^{\Sigma_{P_{k_{0}}^{\dagger}}^{0}\left(w_{k_{0}}^{\dagger}\right)}\left[\frac{\mathbf{e}\left(\mathscr{C}_{k_{0}}: \mathbf{H}-\mathbf{H}_{\mathrm{OO}}: ?\right)}{\prod_{\lambda_{k_{0}}^{\dagger} \in \Sigma_{P_{k_{0}}^{\dagger}}^{0}-\Sigma_{P_{k_{0}}^{+\dagger}}^{0}\left(w_{k_{0}}^{\dagger}\right)}\left(?, \lambda_{k_{0}}^{\dagger}\right)}\right],\right. \\
& \left.D_{*}^{F}\left[\operatorname{tr}_{\mathscr{C}_{k_{0}}}\left(\mathbf{T}_{k_{0} l_{0}}\left(\alpha: \mathbf{H}_{\mathrm{OO}}: ?\right)\right)\right]\right\rangle_{\mathscr{C}_{i}} d \mathfrak{C}\left(\Lambda_{k_{0}}\right) \text {, }
\end{aligned}
$$

both derivatives being evaluated at $\left(1-w_{k_{0}}^{\dagger}\right) \Lambda_{k_{0}}$. All the integral-series are absolutely integrable-summable.

With this result, we are ready to make our final integral manipulation. However, let us first make some notational simplifications. The right hand side of Proposition 9.6 involves $k_{0}$ and $l_{0}$ alone. We can therefore replace $k_{0}$ by $i_{0}$ and $l_{0}$ by $j_{0}$, putting us back where we started 
from. In so doing, it is permissible to change the sum over $w_{k_{0} l_{0}}^{\dagger \prime}=w_{i_{0} j_{0}}^{\dagger \prime}$ to a sum over $w_{j_{0} i_{0}}^{\dagger \prime}$ replacing, then, the inverse $w_{k_{0} l_{0}}^{-\dagger \prime}=w_{i_{0} j_{0}}^{-\dagger \prime}$ by $w_{j_{0} i_{0}}^{\dagger \prime}$, the effect of which is to restore the initial symmetry.

Starting from the beginning, we have

$$
\operatorname{tr}_{\mathscr{E}_{i}}\left(\mathbf{Q}^{\mathbf{I}_{\mathscr{E}_{i}}(\mathbf{H})} \circ \mathbf{L}_{\mathscr{E}_{i} / \Gamma_{\mathscr{E}_{i}}}^{\text {con }}\left(\alpha^{\mathscr{E}_{1}}\right) \circ \mathbf{Q}^{\mathbf{I}_{\mathscr{E}_{i}}(\mathbf{H})}\right)
$$

or still

$$
\frac{1}{(2 \pi)^{l_{l}}} \cdot \int_{\operatorname{Re}\left(\Lambda_{i}\right)=0} \operatorname{tr}\left(\mathbf{Q}_{\mathscr{E}_{i}}^{\mathbf{H}} \circ \mathbf{I n d} \mathbf{I}_{\mathscr{E}_{i}}^{G}\left(\mathbf{L}_{\mathscr{C}_{i} / \Gamma_{\mathscr{E}_{i}}}^{\mathrm{con}} \otimes \Lambda \otimes 1\right)(\alpha) \circ \mathbf{Q}_{\mathscr{E}_{i}}^{\mathbf{H}}\right)\left|d \Lambda_{i}\right|
$$

or still $\left(l_{0}=l_{i}+l_{0}^{\dagger}\right)$

$$
\begin{aligned}
\sum_{\mathscr{E}_{0}^{\dagger}} & \sum_{\mathscr{C}_{i_{0}} \in \mathscr{E}_{0}^{\dagger}} \sum_{\boldsymbol{\theta}_{i_{0}}} \frac{1}{(2 \pi)^{l_{0}}} \cdot \frac{1}{*\left(\mathscr{C}_{i_{0}}^{\dagger}\right)} \\
& \times \int_{\operatorname{Re}\left(\Lambda_{i_{0}}\right)=0} \mathbf{I}_{\alpha}\left(\mathscr{C}_{i}: \boldsymbol{\theta}_{i_{0}}, \Lambda_{i_{0}}: \mathbf{H}\right)\left|d \Lambda_{i_{0}}\right|
\end{aligned}
$$

or still

$$
\begin{aligned}
\int_{\operatorname{Re}\left(\Lambda_{t}\right)=0} \sum_{\mathscr{E}_{0}^{\dagger}} \sum_{\mathscr{E}_{i_{0}} \in \mathscr{E}_{0}^{\dagger}} \sum_{\boldsymbol{O}_{i_{0}}} \frac{1}{(2 \pi)^{l_{0}}} \cdot \frac{1}{*\left(\mathscr{C}_{i_{0}}^{\dagger}\right)} \\
\quad \times \int_{\operatorname{Re}\left(\Lambda_{i_{0}}^{\dagger}\right)=0} \mathbf{I}_{\alpha}\left(\mathscr{C}_{i}: \boldsymbol{O}_{i_{0}}, \Lambda_{i}+\Lambda_{i_{0}}^{\dagger}: \mathbf{H}\right)\left|d \Lambda_{i_{0}}^{\dagger}\right| d \Lambda_{i} \mid
\end{aligned}
$$

or still

$$
\sum_{\mathscr{E}_{10}: \mathbb{E}_{i_{0}}<\mathscr{E}_{i}} \sum_{\boldsymbol{\sigma}_{i_{0}}} \int_{\operatorname{Re}\left(\Lambda_{t_{0}}\right)=0} \mathbf{O}_{\mathscr{E}_{i}}\left(\boldsymbol{\Theta}_{i_{0}}, \Lambda_{i_{0}}: \mathbf{H}\right)\left|d \Lambda_{i_{0}}\right|
$$

plus (cancelling $*\left(\mathscr{C}_{i_{0}}^{\dagger}\right)$ )

$$
\begin{aligned}
& \sum_{\mathscr{E}_{i_{0}}: \mathscr{E}_{i_{0}}\left\langle\mathscr{E}_{1}\right.} \sum_{\boldsymbol{\theta}_{i_{0}}} \frac{(-1)^{l_{0}^{\dagger}}}{(2 \pi)^{l_{0}}} \sum_{w_{i_{0}}^{\dagger}}(-1)^{\#\left(\Sigma_{p_{i_{0}^{\prime \prime}}^{0}}^{0}\left(w_{i_{0}}^{\dagger}\right)\right)} \\
& \times \sum_{F} \frac{1}{C_{i_{0}}(F)} \cdot \sum_{j_{0}} \sum_{w_{j^{\prime} 0_{0}}^{\dagger \prime}} \operatorname{vol}\left(\mathscr{C}_{i^{\prime}}^{\dagger}\right) \\
& \times \int_{\mathfrak{C}\left(\Xi_{1_{0}}\right)}\left\langle D_{F}^{\sum_{P_{10}^{+\prime}}^{0}\left(w_{i_{0}}^{\dagger}\right)}\left[\frac{\mathbf{e}\left(\mathscr{C}_{i_{0}}: \mathbf{H}-\mathbf{H}_{\mathrm{OO}}: ?\right)}{\prod_{\lambda_{t_{0}}^{\dagger} \in \Sigma_{i_{i_{0}}^{\dagger}}^{0}-\Sigma_{P_{t_{0}}^{\dagger}}^{0}\left(w_{t_{0}}^{\dagger}\right)}\left(?, \lambda_{i_{0}}^{\dagger}\right)}\right],\right. \\
& \left.D_{*}^{F}\left[\operatorname{tr}_{\mathscr{C}_{i_{0}}}\left(\mathbf{T}_{i_{0} j_{0}}\left(\alpha: \mathbf{H}_{\mathrm{OO}}: ?\right)\right)\right]\right\rangle_{\mathscr{C}_{1}} d \mathfrak{C}\left(\Lambda_{i_{0}}\right),
\end{aligned}
$$


both derivatives being evaluated at $\left(1-w_{i_{0}}^{\dagger}\right) \Lambda_{i_{0}}$. We explicitly remark that here $\mathbf{T}_{i_{0} j_{0}}\left(\alpha: \mathbf{H}_{\mathrm{OO}}: ?\right)$ is the consecutive composition of

$$
\left\{\begin{array}{l}
\mathbf{c}\left(P_{j_{0}}\left|A_{j_{0}}: P_{i_{0}}\right| A_{i_{0}}: w_{j_{0} i_{0}}^{\dagger \prime} w_{i_{0}}^{\dagger}:-\bar{\Lambda}_{i_{0}}\right)^{*} \\
\mathbf{c}\left(P_{j_{0}}\left|A_{j_{0}}: P_{i_{0}}\right| A_{i_{0}}: w_{j_{0} i_{0}}^{\dagger \prime}: \Lambda_{i_{0}}-?\right) \cdot \mathbf{e}\left(\mathscr{C}_{i_{0}}: \mathbf{H}_{\mathrm{OO}}: ?\right)
\end{array}\right.
$$

and

$$
\operatorname{Ind}_{\mathscr{C}_{i_{0}}}^{G}\left(\left(\boldsymbol{\sigma}_{i_{0}}, \Lambda_{i_{0}}\right)\right)(\alpha) \text {. }
$$

According to what has been said in $\S 8, \bmod o(\mathbf{H})$

$$
\operatorname{tr}\left(Q^{\mathbf{H}} \circ L_{G / \Gamma}^{\mathrm{dis}}(\alpha) \circ Q^{\mathbf{H}}\right)
$$

is the same as

$$
\mathbf{K}\left(\mathbf{H}_{O}: \alpha: \Gamma\right)
$$

less

$$
\begin{aligned}
& \sum_{\mathscr{E}_{1}}(-1)^{\mathrm{rank}\left(\mathscr{E}_{1}\right)}
\end{aligned}
$$

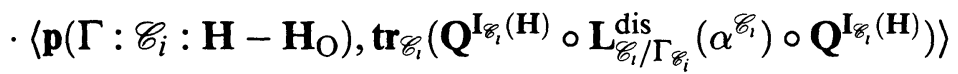

less

$$
\begin{aligned}
& \sum_{\mathscr{C}_{i}}(-1)^{\operatorname{rank}\left(\mathscr{C}_{i}\right)} \\
& \cdot\left\langle\mathbf{p}\left(\Gamma: \mathscr{C}_{i}: \mathbf{H}-\mathbf{H}_{\mathrm{O}}\right), \mathbf{t r}_{\mathscr{C}_{i}}\left(\mathbf{Q}^{\mathbf{I}_{\mathscr{E}_{i}}(\mathbf{H})} \circ \mathbf{L}_{\mathscr{C}_{1} / \Gamma_{\mathscr{E}_{i}}}^{\text {con }}\left(\alpha^{\mathscr{C}_{1}}\right) \circ \mathbf{Q}^{\mathbf{I}_{\mathscr{E}_{i}}(\mathbf{H})}\right)\right\rangle .
\end{aligned}
$$

The discussion in the preceding paragraph was conducted under the assumption that $\mathscr{C}_{i_{0}} \prec \mathscr{C}_{i}$. Allowing $\mathscr{C}_{i_{0}}=\mathscr{C}_{i}\left(\mathscr{C}_{i} \neq\{G\}\right)$ simply means that the corresponding $L^{\text {dis }}$ terms must also be included. So, $\bmod \mathbf{o}(\mathbf{H})$

$$
\operatorname{tr}\left(Q^{\mathbf{H}} \circ L_{G / \Gamma}^{\text {dis }}(\alpha) \circ Q^{\mathbf{H}}\right)
$$

is the same as

$$
\mathbf{K}\left(\mathbf{H}_{\mathrm{O}}: \alpha: \Gamma\right)
$$

less (absorbing $\left.(-1)^{\operatorname{rank}\left(\mathscr{C}_{l}\right)}\right)$

$$
\sum_{\mathscr{C}_{i}, \mathscr{C}_{i_{0}}} \sum_{\boldsymbol{O}_{i_{0}}} \int_{\operatorname{Re}\left(\Lambda_{i_{0}}\right)=0}\left\langle\mathbf{p}\left(\Gamma: \mathscr{C}_{i}: \mathbf{H}-\mathbf{H}_{\mathrm{O}}\right), \mathbf{o}_{\mathscr{C}_{1}}\left(\boldsymbol{\sigma}_{i_{0}}, \Lambda_{i_{0}}: \mathbf{H}\right)\right\rangle\left|d \Lambda_{i_{0}}\right|
$$


less $\left(l_{i}=\operatorname{rank}\left(\mathscr{C}_{i}\right), l_{0}=l_{i}+l_{0}^{\dagger}\right)$

$$
\begin{aligned}
& \sum_{\mathscr{C}_{1}, \mathscr{B}_{i_{0}}} \sum_{\boldsymbol{\sigma}_{i_{0}}} \frac{(-1)^{l_{0}}}{(2 \pi)^{l_{0}}} \sum_{w_{i_{0}}^{\dagger}}(-1)^{\#\left(\Sigma_{P_{i_{0}}^{\dagger}}^{0}\left(w_{i_{0}}^{\dagger}\right)\right)} \\
& \times \sum_{F} \frac{1}{C_{i_{0}}(F)} \cdot \sum_{j_{0}} \sum_{w_{j^{\prime} 0}^{\dagger \prime}} \operatorname{vol}\left(\mathscr{C}_{i^{\prime}}^{\dagger}\right) \\
& \times \int_{\mathfrak{C}\left(\Xi_{t_{0}}\right)}\left\langle\mathbf{p}\left(\Gamma: \mathscr{C}_{i}: \mathbf{H}-\mathbf{H}_{\mathrm{O}}\right),\right. \\
& \left\langle D_{F}^{\Sigma_{P_{i_{0}}^{\dagger}}^{0}\left(w_{i_{0}}^{\dagger}\right)}\left[\frac{\mathbf{e}\left(\mathscr{C}_{i_{0}}: \mathbf{H}-\mathbf{H}_{\mathrm{OO}}: ?\right)}{\prod_{\lambda_{i_{0}}^{\dagger} \in \Sigma_{P_{i_{0}}^{\dagger}}^{0}-\Sigma_{P_{i_{0}}^{\prime \prime}}^{0}\left(w_{t_{0}}^{\dagger}\right)}\left(?, \lambda_{i_{0}}^{\dagger}\right)}\right],\right. \\
& \left.\left.D_{*}^{F}\left[\operatorname{tr}_{\mathscr{C}_{i_{0}}}\left(\mathbf{T}_{i_{0} j_{0}}\left(\alpha: \mathbf{H}_{\mathrm{OO}}: ?\right)\right)\right]\right\rangle_{\mathscr{C}_{1}}\right\rangle d \mathfrak{C}\left(\Lambda_{i_{0}}\right),
\end{aligned}
$$

the outside pairing being one of $r_{i}$-tuples. Here, the sum $\sum_{\mathscr{C}_{1}, \mathscr{C}_{i_{0}}}$ extends over all $\mathscr{C}_{i}, \mathscr{C}_{i_{0}}$ such that $\mathscr{C}_{i} \succeq \mathscr{C}_{i_{0}}\left(\mathscr{C}_{i_{0}} \neq\{G\}\right)$. Needless to say, the derivatives are again evaluated at $\left(1-w_{i_{0}}^{\dagger}\right) \Lambda_{i_{0}}$.

In conclusion:

THEOREM 9.7. Mod o(H)

$$
\operatorname{tr}\left(Q^{\mathbf{H}} \circ L_{G / \Gamma}^{\mathrm{dis}}(\alpha) \circ Q^{\mathbf{H}}\right)
$$

is the same as

$$
\mathbf{K}\left(\mathbf{H}_{\mathrm{O}}: \alpha: \Gamma\right)
$$

less

$$
\sum_{\mathscr{E}_{i}, \mathscr{C}_{i_{0}}} \sum_{\mathscr{\sigma}_{i_{0}}} \int_{\operatorname{Re}\left(\Lambda_{i_{0}}\right)=0}\left\langle\mathbf{p}\left(\Gamma: \mathscr{C}_{i}: \mathbf{H}-\mathbf{H}_{\mathrm{O}}\right), \mathbf{o}_{\mathscr{C}_{i}}\left(\boldsymbol{\sigma}_{i_{0}}, \Lambda_{i_{0}}: \mathbf{H}\right)\right\rangle\left|d \Lambda_{i_{0}}\right|
$$


less

$$
\begin{aligned}
& \sum_{\mathscr{C}_{i}, \mathscr{G}_{i_{0}}} \sum_{\boldsymbol{\sigma}_{i_{0}}} \frac{(-1)^{l_{0}}}{(2 \pi)^{l_{0}}} \sum_{w_{i_{0}}^{\dagger}}(-1)^{\#\left(\Sigma_{i_{i_{0}}^{\dagger}}^{0}\left(w_{i_{0}}^{\dagger}\right)\right)} \\
& \times \sum_{F} \frac{(-1)^{\#(F)}}{C_{i_{0}}(F)} \cdot \sum_{j_{0}} \sum_{w_{j_{0} t_{0}}^{\dagger \prime}} \operatorname{vol}\left(\mathscr{C}_{i^{\prime}}^{\dagger}\right) \\
& \times \int_{\mathfrak{C}\left(\Xi_{i_{0}}\right)}\left\langle\mathbf{p}\left(\Gamma: \mathscr{C}_{i}: \mathbf{H}-\mathbf{H}_{\mathrm{O}}\right),\right. \\
& \left\langle D_{F}^{\Sigma_{P_{t_{0}}^{\dagger}}^{0}\left(w_{i_{0}}^{\dagger}\right)}\left[\frac{\mathbf{e}\left(\mathscr{C}_{i_{0}}: \mathbf{H}-\mathbf{H}_{\mathrm{OO}}: ?\right)}{\prod_{\lambda_{t_{0}}^{\dagger} \in \Sigma_{P_{i_{0}}^{\dagger}}^{0}-\Sigma_{P_{i_{0}}^{\dagger}}^{0}\left(w_{i_{0}}^{\dagger}\right)}\left(?, \lambda_{i_{0}}^{\dagger}\right)}\right]_{\left(1-w_{i_{0}}^{\dagger}\right) \Lambda_{t_{0}}},\right. \\
& \operatorname{tr}_{\mathscr{E}_{i_{0}}}\left\{\mathbf{c}\left(P_{j_{0}}\left|A_{j_{0}}: P_{i_{0}}\right| A_{i_{0}}: w_{j_{0} i_{0}}^{\dagger \prime} w_{i_{0}}^{\dagger}:-\bar{\Lambda}_{i_{0}}\right)^{*}\right. \\
& \times D_{*}^{F}\left[\mathbf{c}\left(P_{j_{0}}\left|A_{j_{0}}: P_{i_{0}}\right| A_{i_{0}}: w_{j_{0} i_{0}}^{\dagger \prime}: ?\right) \cdot \mathbf{e}\left(\mathscr{C}_{i_{0}}: \mathbf{H}_{\mathrm{OO}}: ?\right)\right]_{w_{i_{0}}^{\dagger} \Lambda_{i_{0}}} \\
& \left.\left.\left.\times \operatorname{Ind}_{\mathscr{C}_{l_{0}}}^{G}\left(\left(\boldsymbol{\Theta}_{i_{0}}, \Lambda_{i_{0}}\right)\right)(\alpha)\right\}\right\rangle_{\mathscr{C}_{\imath}}\right\rangle d \mathfrak{C}\left(\Lambda_{i_{0}}\right) .
\end{aligned}
$$

10. Two examples. The purpose of this section is to illustrate how the cancellation works in the two simplest cases which, in a sense, are the exact opposite to on' another.

The preceding theorem gives us an expression for

$$
\operatorname{tr}\left(Q^{\mathbf{H}} \circ L_{G / \Gamma}^{\mathrm{dis}}(\alpha) \circ Q^{\mathbf{H}}\right)
$$

containing three distinct ingredients. The first of these,

$$
\mathbf{K}\left(\mathbf{H}_{\mathrm{O}}: \alpha: \Gamma\right),
$$

will be left as is since it contains the contribution to

$$
\operatorname{tr}\left(Q^{\mathbf{H}} \circ L_{G / \Gamma}^{\mathrm{dis}}(\alpha) \circ Q^{\mathbf{H}}\right)
$$

associated with the conjugacy classes and will be studied elsewhere. As for the second,

$$
\sum_{\mathscr{C}_{1}, \mathscr{C}_{i_{0}}} \sum_{\boldsymbol{\sigma}_{i_{0}}} \int_{\operatorname{Re}\left(\Lambda_{i_{0}}\right)=0}\left\langle\mathbf{p}\left(\Gamma: \mathscr{C}_{i}: \mathbf{H}-\mathbf{H}_{\mathrm{O}}\right), \mathbf{o}_{\mathscr{C}_{i}}\left(\boldsymbol{\sigma}_{i_{0}}, \Lambda_{i_{0}}: \mathbf{H}\right)\right\rangle\left|d \Lambda_{i_{0}}\right|
$$


it turns out to be $\mathbf{o}(\mathbf{H})$, so, for all intents and purposes, can be ignored. We shall justify this assertion in detail eventually. Consequently, in what follows, our chief concern is with the third term.

There is, however, a little more to be said before we try to cut our way through this thicket. Write

$$
\sum_{\mathscr{C}_{1}, \mathscr{C}_{10}}=\sum_{\mathscr{C}_{10}} \sum_{\mathscr{C}_{1}: \mathscr{C}_{1} \succeq \mathscr{C}_{1_{0}}}
$$

Then:

LEMMA 10.1.

$$
\sum_{\mathscr{E}_{1}: \mathscr{E}_{1} \succeq \mathscr{C}_{1_{0}}} \sum_{w_{t_{0}}^{\dagger}}=\sum_{w_{i_{0}}} \sum_{\mathscr{E}_{1}: \mathscr{E}_{1} \succeq \mathscr{C}_{1}\left(w_{i_{0}}\right)}
$$

The notation is intended to be indicative: $\forall w_{i_{0}} \mathscr{C}_{i}\left(w_{i_{0}}\right)$ is the $G$ conjugacy class determined by

$$
\left\{\lambda_{i_{0}} ; w_{i_{0}} \lambda^{i_{0}} \neq \lambda^{i_{0}}\right\}
$$

whereas the dual of the split component of the parabolic $P_{i}\left(w_{i_{0}}\right) \succeq P_{i_{0}}$ is

$$
\operatorname{span}\left\{\lambda^{i_{0}}: w_{i_{0}} \lambda^{i_{0}}=\lambda^{i_{0}}\right\} .
$$

While strictly elementary, the lemma is important in that it does serve to characterize the relevant $\mathscr{C}_{i}$ as those lying above a fixed "base class" depending on the choice of $w_{i_{0}}$.

Accordingly,

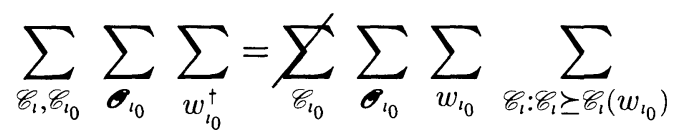

To profit from this change, recall that the Arthur polynomial $\mathbf{p}\left(\Gamma: \mathscr{C}_{i}: ?\right)$ is homogeneous of degree $l_{i}\left(=\operatorname{rank}\left(\mathscr{C}_{i}\right)\right)$, hence

$$
\mathbf{p}\left(\Gamma: \mathscr{C}_{i}: \mathbf{H}-\mathbf{H}_{\mathrm{O}}\right)=(-1)^{l_{i}} \cdot \mathbf{p}\left(\Gamma: \mathscr{C}_{i}: \mathbf{H}_{\mathrm{O}}-\mathbf{H}\right) .
$$

Moreover,

$$
\Sigma_{P_{t_{0}}^{+\prime}}^{0}\left(w_{i_{0}}\right)=\Sigma_{P_{t_{0}}}^{0}\left(w_{i_{0}}\right) \cap \Sigma_{P_{t_{0}}^{\dagger}}^{0}
$$

implying that

$$
\#\left(\Sigma_{P_{i_{0}}^{\dagger \prime}}^{0}\left(w_{i_{0}}\right)\right)=\#\left(\Sigma_{P_{i_{0}}}^{0}\left(w_{i_{0}}\right)\right)+l_{0}^{\dagger}-\#\left(\Sigma_{P_{i_{0}}}^{0}\left(w_{i_{0}}\right) \cup \Sigma_{P_{i_{0}}^{\dagger}}^{0}\right),
$$

from which

$$
(-1)^{l_{0}^{\dagger}+\#\left(\Sigma_{P_{t_{0}}^{\dagger}}^{0}\left(w_{t_{0}}^{\dagger}\right)\right)}=(-1)^{\#\left(\Sigma_{P_{t_{0}}^{\dagger}}^{0}-\Sigma_{P_{t_{0}}}^{0}\left(w_{t_{0}}\right)\right)} .
$$


We must therefore examine

$$
\begin{aligned}
& \sum_{\mathscr{E}_{l_{0}}} \sum_{\boldsymbol{O}_{i_{0}}} \frac{1}{(2 \pi)^{l_{0}}} \sum_{w_{i_{0}}} \sum_{\mathscr{C}_{l}: \mathscr{C}_{l} \succeq \mathscr{C}_{l}\left(w_{l_{0}}\right)}(-1)^{\#\left(\Sigma_{P_{i_{0}}^{\dagger}}^{0}-\Sigma_{P_{i_{0}}}^{0}\left(w_{\imath_{0}}\right)\right)} \\
& \times \sum_{F} \frac{(-1)^{\#(F)}}{C_{i_{0}}(F)} \cdot \sum_{j_{0}} \sum_{w_{J_{0} i_{0}}} \operatorname{vol}\left(\mathscr{C}_{i^{\prime}}^{\dagger}\right) \\
& \times \int_{\mathfrak{C}\left(\Xi_{t_{0}}\right)}\left\langle\mathbf{p}\left(\Gamma: \mathscr{C}_{i}: \mathbf{H}_{\mathrm{O}}-\mathbf{H}\right),\right. \\
& \left\langle D_{F}^{\Sigma_{P_{t_{0}^{\prime}}^{\prime}}^{0}\left(w_{t_{0}}\right)}\left[\frac{\mathbf{e}\left(\mathscr{C}_{i_{0}}: \mathbf{H}-\mathbf{H}_{\mathrm{OO}}: ?\right)}{\prod_{\lambda_{t_{0}}^{\dagger} \in \Sigma_{P_{i_{0}}^{\dagger}}^{0}-\Sigma_{P_{t_{0}}^{\dagger}}^{0}\left(w_{t_{0}}\right)}\left(?, \lambda_{i_{0}}^{\dagger}\right)}\right]_{\left(1-w_{t_{0}}\right) \Lambda_{i_{0}}},\right. \\
& \operatorname{tr}_{\mathscr{C}_{i_{0}}}\left\{\mathbf{c}\left(P_{j_{0}}\left|A_{j_{0}}: P_{i_{0}}\right| A_{i_{0}}: w_{j_{0} i_{0}} w_{i_{0}}:-\bar{\Lambda}_{i_{0}}\right)^{*}\right. \\
& \times D_{*}^{F}\left[\mathbf{c}\left(P_{j_{0}}\left|A_{j_{0}}: P_{i_{0}}\right| A_{i_{0}}: w_{j_{0} i_{0}}: ?\right) \cdot \mathbf{e}\left(\mathscr{C}_{i_{0}}: \mathbf{H}_{\mathrm{OO}}: ?\right)\right]_{w_{t_{0}} \Lambda_{t_{0}}} \\
& \left.\left.\left.\times \operatorname{Ind}_{\mathscr{C}_{i_{0}}}^{G}\left(\left(\boldsymbol{\theta}_{i_{0}}, \Lambda_{i_{0}}\right)\right)(\alpha)\right\}\right\rangle_{\mathscr{C}_{i}}\right\rangle d \mathfrak{C}\left(\Lambda_{i_{0}}\right) .
\end{aligned}
$$

[Note: It is a question now of $w_{j_{0} i_{0}}$ and not of $w_{j_{0} i_{0}}^{\dagger \prime}$. Of course, the set over which $w_{j_{0} i_{0}}$ is summed depends on $F$.]

REMARK. For use below, it will be necessary to keep in mind that $\operatorname{vol}\left(\mathscr{C}_{i^{\prime}}^{\dagger}\right)$ is the volume of the projection onto

$$
\operatorname{span}\left(\Sigma_{P_{i_{0}}^{\dagger \prime}}^{0}\left(w_{i_{0}}\right)\right)^{\perp}
$$

of the parallelepiped with sides corresponding to the elements of

$$
\Sigma_{P_{i_{0}}^{\dagger}}^{0}-\Sigma_{P_{i_{0}}^{\dagger \prime}}^{0}\left(w_{i_{0}}\right) \text {. }
$$

Given $\mathscr{C}_{i_{0}}$, we determine $P_{i_{0}}$ and $A_{i_{0}}$ and thus the set $W\left(A_{i_{0}}\right)$ comprised of the various $w_{i_{0}}$. Our limit will depend on

$$
\operatorname{rank}\left(1-w_{i_{0}}\right) \text {. }
$$

To get a feel for what happens, let us consider by way of illustration the two extreme possibilities, viz. when

$$
\left\{\begin{array}{l}
\operatorname{rank}\left(1-w_{i_{0}}\right)=0, \\
\operatorname{rank}\left(1-w_{i_{0}}\right)=l_{0},
\end{array}\right.
$$

respectively. It is quite remarkable that, depending on the case, totally different methods are needed in order to carry out the evaluation. 
EXAMPLE. Suppose that $\operatorname{rank}\left(1-w_{i_{0}}\right)=0$-then $w_{i_{0}}=1, \mathscr{C}_{i}\left(w_{i_{0}}\right)=$ $\mathscr{C}_{i_{0}}$ and

$$
\left\{\begin{array}{l}
\Sigma_{P_{i_{0}}}^{0}\left(w_{i_{0}}\right)=\Sigma_{P_{i_{0}}}^{0}, \\
\Sigma_{P_{i_{0}}^{\dagger}}^{0}\left(w_{i_{0}}\right)=\Sigma_{P_{i_{0}}^{+}}^{0}
\end{array}\right.
$$

So, in this situation, $\sum_{F}$ is the sum over the $F \subset \Sigma_{P_{i_{0}}^{\dagger}}^{0}$, the product denominator is unity and $\operatorname{vol}\left(\mathscr{C}_{i^{\prime}}^{\dagger}\right)=1$. That being the case, we can pass directly from $\mathfrak{C}\left(\Xi_{i_{0}}\right)$ to $\operatorname{Re}\left(\Lambda_{i_{0}}\right)=0$, obtaining

$$
\begin{aligned}
& \sum_{\mathscr{C}_{1}: \mathscr{G}_{1} \succeq \mathscr{C}_{1}\left(w_{\imath_{0}}\right)} \sum_{F} \frac{(-1)^{\#(F)}}{C_{i_{0}}(F)} \cdot \sum_{j_{0}} \sum_{w_{j_{0} t_{0}}} \\
& \times \int_{\operatorname{Re}\left(\Lambda_{t_{0}}\right)=0}\left\langle\mathbf{p}\left(\Gamma: \mathscr{C}_{i}: \mathbf{H}_{\mathrm{O}}-\mathbf{H}\right),\right. \\
& \left.\left\langle D_{F}^{\Sigma_{P^{\dagger}}^{0}}\left[\mathbf{e}\left(\mathscr{C}_{i_{0}}: \mathbf{H}-\mathbf{H}_{\mathrm{OO}}: ?\right)\right]_{0}, \mathbf{t r} \mathscr{\mathscr { C }}_{i_{0}}\{\cdots\}\right\rangle_{\mathscr{C}_{i}}\right\rangle\left|d \Lambda_{i_{0}}\right|
\end{aligned}
$$

Putting

$$
\mathbf{p}_{F}^{\Sigma_{P_{i_{0}}^{\dagger}}^{0}}(\mathbf{H})=D_{F}^{\Sigma_{P_{t_{0}}^{\dagger}}^{0}}\left[\mathbf{e}\left(\mathscr{C}_{i_{0}}: \mathbf{H}: ?\right)\right]_{0},
$$

interchange the order of summation in $\mathscr{C}_{i}$ and $F$ and then pull the summation in $\mathscr{C}_{i}$ all the way to the right to get

$$
\begin{aligned}
& \sum_{F} \frac{(-1)^{\#(F)}}{C_{i_{0}}(F)} \cdot \sum_{j_{0}} \sum_{w_{J^{\prime} t_{0}}} \\
& \quad \times \int_{\operatorname{Re}\left(\Lambda_{i_{0}}\right)=0} \sum_{\mathscr{C}_{i}: \mathscr{C}_{i} \succeq \mathscr{C}_{i_{0}}(F)}\left\langle\mathbf{p}\left(\Gamma: \mathscr{C}_{i}: \mathbf{H}_{\mathrm{O}}-\mathbf{H}\right),\right. \\
& \left.\left\langle\mathbf{p}_{F}^{\Sigma_{P^{\dagger}}^{\dagger}}\left(\mathbf{H}-\mathbf{H}_{\mathrm{OO}}\right), \operatorname{tr}_{\mathscr{C}_{i_{0}}}\{\cdots\}\right\rangle \mathscr{\mathscr { C }}_{i}\right\rangle\left|d \Lambda_{i_{0}}\right|
\end{aligned}
$$

or still, using the reduction maps and Proposition 3.2,

$$
\begin{aligned}
& \sum_{F} \frac{(-1)^{\#(F)}}{C_{i_{0}}(F)} \cdot \sum_{j_{0}} \sum_{w_{J_{0} i_{0}}} \\
& \quad \times \int_{\operatorname{Re}\left(\Lambda_{i_{0}}\right)=0}\left\langle\mathbf{p}\left(\Gamma: \mathscr{C}_{i_{0}}(F): \mathbf{H}_{\mathrm{O}}-\mathbf{H}_{\mathrm{OO}}\right), \mathbf{t r}_{\mathscr{C}_{i_{0}}(F)}\{\cdots\}\right\rangle\left|d \Lambda_{i_{0}}\right| .
\end{aligned}
$$

Here, $\operatorname{tr}_{\mathscr{G}_{t_{0}}(F)}\{\cdots\}$ at $\Lambda_{i_{0}}$ is

$$
\begin{aligned}
& \operatorname{tr}_{\mathscr{G}_{i_{0}}(F)}\left\{\mathbf{c}\left(P_{j_{0}}\left|A_{j_{0}}: P_{i_{0}}\right| A_{i_{0}}: w_{j_{0} i_{0}}: \Lambda_{i_{0}}\right)^{*}\right. \\
& \times D_{*}^{F}\left[\mathbf{c}\left(P_{j_{0}}\left|A_{j_{0}}: P_{i_{0}}\right| A_{i_{0}}: w_{j_{0} i_{0}}: \Lambda_{i_{0}}\right) \cdot \mathbf{e}\left(\mathscr{C}_{i_{0}}: \mathbf{H}_{\mathrm{OO}}: \Lambda_{i_{0}}\right)\right] \\
& \left.\times \operatorname{Ind}_{\mathscr{C}_{i_{0}}}^{G}\left(\left(\boldsymbol{\theta}_{i_{0}}, \Lambda_{i_{0}}\right)\right)(\alpha)\right\} \text {. }
\end{aligned}
$$


Because $\mathbf{H}$ has now been eliminated, the cancellation vis-à-vis $w_{i_{0}}=1$ is complete. Of course, we have yet to sum over the $\sigma_{i_{0}}$, a triviality. Incidentally, the term attached to $F=\Sigma_{P_{i_{0}}^{\dagger}}^{0}$ will appear in the trace formula itself.

[Note: For a check, take $\operatorname{rank}(\Gamma)=1$-then, taking $\mathbf{H}_{\mathrm{OO}}=0$, as things are in reality subtracted, what ultimately survives is

$$
\begin{aligned}
-\frac{(-1)}{4 \pi} \cdot \sum_{\boldsymbol{\theta}_{i_{0}}} \int_{\operatorname{Re}\left(\Lambda_{i_{0}}\right)=0} \operatorname{tr} & \left\{\operatorname{Ind}_{\mathscr{G}_{i_{0}}}^{G}\left(\left(\boldsymbol{\sigma}_{i_{0}}, \Lambda_{i_{0}}\right)\right)(\alpha)\right. \\
& \times \mathbf{c}\left(P_{i_{0}}\left|A_{i_{0}}: P_{i_{0}}\right| A_{i_{0}}: w_{i_{0}}: \Lambda_{i_{0}}\right)^{*} \\
& \left.\cdot D_{*}^{*}\left[\mathbf{c}\left(P_{i_{0}}\left|A_{i_{0}}: P_{i_{0}}\right| A_{i_{0}}: w_{i_{0}}: \Lambda_{i_{0}}\right)\right]\right\}\left|d \Lambda_{i_{0}}\right|
\end{aligned}
$$

i.e., since $D_{*}^{*}=-d / d \Lambda_{i_{0}}($ in $\operatorname{rank} 1)$,

$$
\begin{aligned}
&-\frac{1}{4 \pi} \cdot \sum_{\boldsymbol{\sigma}_{i_{0}}} \int_{\operatorname{Re}\left(\Lambda_{i_{0}}\right)=0} \operatorname{tr}\left\{\operatorname{Ind}_{\mathscr{G}_{i_{0}}}^{G}\left(\left(\boldsymbol{\sigma}_{i_{0}}, \Lambda_{i_{0}}\right)\right)(\alpha)\right. \\
& \quad \times \mathbf{c}\left(P_{i_{0}}\left|A_{i_{0}}: P_{i_{0}}\right| A_{i_{0}}: w_{i_{0}}: \Lambda_{i_{0}}\right)^{*} \\
&\left.\cdot \frac{d}{d \Lambda_{i_{0}}} \mathbf{c}\left(P_{i_{0}}\left|A_{i_{0}}: P_{i_{0}}\right| A_{i_{0}}: w_{i_{0}}: \Lambda_{i_{0}}\right)\right\}\left|d \Lambda_{i_{0}}\right|
\end{aligned}
$$

It should be pointed out that the minus sign prefacing the $1 / 4 \pi$ in the last expression corrects an error made initially by Arthur (in his $F$-rank 1 paper) and then again by Osborne and Warner [2-(a), p. 51].]

EXAMPLE. Suppose that $\operatorname{rank}\left(1-w_{i_{0}}\right)=l_{0}$-then $1-w_{i_{0}}$ is nonsingular, $\mathscr{C}_{i}\left(w_{i_{0}}\right)=\{G\}$ and

$$
\left\{\begin{array}{l}
\Sigma_{P_{t_{0}}}^{0}\left(w_{i_{0}}\right)=\varnothing \\
\Sigma_{P_{t_{0}}^{\dagger}}^{0}\left(w_{i_{0}}\right)=\varnothing
\end{array}\right.
$$

So, in this situation, $\sum_{F}$ is the sum over singleton $F=\varnothing$, the product denominator is over all the $\lambda_{i_{0}} \in \Sigma_{P_{t_{0}}}^{0}$ and

$$
\operatorname{vol}\left(\mathscr{C}_{i^{\prime}}^{\dagger}\right)=\operatorname{vol}\left(\mathscr{C}_{i_{0}}\right)
$$

Additionally, $j_{0}=i_{0}, w_{j_{0} i_{0}}=1$ and there is no differentiation. Noting that

$$
\left\{\begin{array}{l}
\mathbf{p}\left(\Gamma:\{G\}: \mathbf{H}_{\mathrm{OO}}-\mathbf{H}\right)=1 \\
\left\langle\mathbf{1}, \operatorname{tr}_{\mathscr{C}_{I_{0}}}\{\cdots\}\right\rangle_{\{G\}}=\operatorname{tr}_{\{G\}}\{\cdots\}=\operatorname{tr}\{\cdots\}
\end{array}\right.
$$


we are reduced to calculating

$$
\begin{aligned}
\operatorname{vol}\left(\mathscr{C}_{i_{0}}\right) \cdot \int_{\mathfrak{C}\left(\Xi_{i_{0}}\right)} \operatorname{tr}\left\{\frac{\mathbf{e}\left(\mathscr{C}_{i_{0}}: \mathbf{H}-\mathbf{H}_{\mathrm{OO}}:\left(1-w_{i_{0}}\right) \Lambda_{i_{0}}\right)}{\prod_{\lambda_{i_{0}}}\left(\left(1-w_{i_{0}}\right) \Lambda_{i_{0}}, \lambda_{i_{0}}\right)}\right. \\
\times \mathbf{c}\left(P_{i_{0}}\left|A_{i_{0}}: P_{i_{0}}\right| A_{i_{0}}: w_{i_{0}}:-\bar{\Lambda}_{i_{0}}\right)^{*} \\
\left.\cdot \mathbf{I n d}_{\mathscr{C}_{i_{0}}}^{G}\left(\left(\boldsymbol{\sigma}_{i_{0}}, \Lambda_{i_{0}}\right)\right)(\alpha)\right\} d \mathfrak{C}\left(\Lambda_{i_{0}}\right)
\end{aligned}
$$

or still

$$
\begin{aligned}
& \operatorname{vol}\left(\mathscr{C}_{i_{0}}\right) \cdot \int_{\mathfrak{C}\left(\Xi_{i_{0}}\right)} \frac{1}{\prod_{\lambda_{i_{0}}}\left(\left(1-w_{i_{0}}\right) \Lambda_{i_{0}}, \lambda_{i_{0}}\right)} \\
& \quad \times \operatorname{tr}\left\{\mathbf{e}\left(\mathscr{C}_{i_{0}}: \mathbf{H}-\mathbf{H}_{\mathrm{OO}}:\left(1-w_{i_{0}}\right) \Lambda_{i_{0}}\right) \cdot \mathbf{f}\left(\left(1-w_{i_{0}}\right) \Lambda_{i_{0}}\right)\right\} d \mathfrak{C}\left(\Lambda_{i_{0}}\right)
\end{aligned}
$$

if

$$
\begin{aligned}
\mathbf{f}\left(\Lambda_{i_{0}}\right)= & \mathbf{c}\left(P_{i_{0}}\left|A_{i_{0}}: P_{i_{0}}\right| A_{i_{0}}: w_{i_{0}}:-\left(1-w_{i_{0}}\right)^{-1} \bar{\Lambda}_{i_{0}}\right)^{*} \\
& \cdot \text { Ind }_{\mathscr{C}_{i_{0}}}^{G}\left(\left(\boldsymbol{\sigma}_{i_{0}},\left(1-w_{i_{0}}\right)^{-1} \Lambda_{i_{0}}\right)\right)(\alpha) .
\end{aligned}
$$

The form of the integrand suggests that a change of variables is in order:

$$
\Lambda_{i_{0}} \rightarrow\left(1-w_{i_{0}}\right) \Lambda_{i_{0}}
$$

Suppressing the formalities, we get

$$
\begin{aligned}
& \frac{\operatorname{vol}\left(\mathscr{C}_{i_{0}}\right)}{\left|\operatorname{det}\left(1-w_{i_{0}}\right)\right|} \cdot \int_{\mathfrak{C}\left(\left(1-w_{i_{0}}\right) \Xi_{i_{0}}\right)} \frac{1}{\prod_{\lambda_{t_{0}}}\left(\Lambda_{i_{0}}, \lambda_{i_{0}}\right)} \\
& \quad \times \operatorname{tr}\left\{\mathbf{e}\left(\mathscr{C}_{i_{0}}: \mathbf{H}-\mathbf{H}_{\mathrm{OO}}: \Lambda_{i_{0}}\right) \cdot \mathbf{f}\left(\Lambda_{i_{0}}\right)\right\} d \mathfrak{C}\left(\Lambda_{i_{0}}\right),
\end{aligned}
$$

where

$$
\left(1-w_{i_{0}}\right) \Xi_{i_{0}}=\left\{\frac{\left(1-w_{i_{0}}\right) \xi_{i_{0}}}{\left\|\left(1-w_{i_{0}}\right) \xi_{i_{0}}\right\|}: \xi_{i_{0}} \in \Xi_{i_{0}}\right\}
$$

It now follows from the elements of the Dini calculus (or directly) that the preceding integral is

$$
\frac{1}{\left|\operatorname{det}\left(1-w_{i_{0}}\right)\right|} \cdot c\left(w_{i_{0}}\right)(-2 \pi)^{l_{0}} \operatorname{tr}(\mathbf{f}(0))+\mathbf{o}(\mathbf{H}) \quad(\mathbf{H} \rightarrow-\infty) .
$$

Here,

$$
c\left(w_{i_{0}}\right)=\frac{\#\left\{\xi_{i_{0}} \in \Xi_{i_{0}}:\left(1-w_{i_{0}}\right) \xi_{i_{0}} \in-\mathscr{C}_{P_{i_{0}}}\right\}}{\#\left(\Xi_{i_{0}}\right)} .
$$

Since we are not interested in those entities that are $\mathbf{o}(\mathbf{H})$, we finally end up with

$$
\frac{1}{\left|\operatorname{det}\left(1-w_{i_{0}}\right)\right|} \cdot c\left(w_{i_{0}}\right)(-2 \pi)^{l_{0}} \cdot \operatorname{tr}(\mathbf{f}(0))
$$


or still, bringing in the $1 /(2 \pi)^{l_{0}}$ (which is out in front) and the $(-1)^{l_{0}}$ (which is part of the data), upon explication,

$$
\begin{aligned}
c\left(w_{i_{0}}\right) & \cdot \frac{1}{\left|\operatorname{det}\left(1-w_{i_{0}}\right)\right|} \\
& \cdot \operatorname{tr}\left\{\mathbf{c}\left(P_{i_{0}}\left|A_{i_{0}}: P_{i_{0}}\right| A_{i_{0}}: w_{i_{0}}: 0\right) \cdot \mathbf{I n d}_{\mathscr{G}_{i_{0}}}^{G}\left(\left(\boldsymbol{\sigma}_{i_{0}}, 0\right)\right)(\alpha)\right\} .
\end{aligned}
$$

Observe that $\mathbf{H}_{\mathrm{O}}$ is nowhere to be found. Accordingly, summing these terms over $\theta_{i_{0}}$ gives the contribution to the trace formula arising from the nonsingular $1-w_{i_{0}}$.

[Note: For a check, take $\operatorname{rank}(\Gamma)=1$-then, as things are in reality subtracted, what ultimately survives is

$$
-\frac{1}{4} \cdot \sum_{\boldsymbol{\sigma}_{i_{0}}} \operatorname{tr}\left\{\mathbf{c}\left(\boldsymbol{P}_{i_{0}}: A_{i_{0}} \mid P_{i_{0}}: A_{i_{0}}: w_{i_{0}}: 0\right) \cdot \mathbf{I n d}_{\mathscr{C}_{i_{0}}}^{G}\left(\left(\boldsymbol{\sigma}_{i_{0}}, 0\right)\right)(\alpha)\right\},
$$

the extra 2 coming from the relation

$$
\left|\operatorname{det}\left(1-w_{i_{0}}\right)\right|=2 \text {, }
$$

valid in rank 1. It is reassuring that this expression does agree with the one derived earlier by us (cf. [2-(a), p. 51]).]

At first glance, the nature of the $c\left(w_{i_{0}}\right)$ figuring in the foregoing may seem to be a bit opaque. However, the situation can be clarified in that actually

$$
\begin{aligned}
\sum_{\mathscr{E}_{10} \in \mathscr{C}_{0}} & \sum_{w_{i_{0}}: \operatorname{det}\left(1-w_{i_{0}}\right) \neq 0} c\left(w_{i_{0}}\right) \cdot \frac{1}{\left|\operatorname{det}\left(1-w_{i_{0}}\right)\right|} \\
= & \sum_{\mathscr{C}_{i_{0}} \in \mathscr{C}_{0}} \sum_{w_{i_{0}}: \operatorname{det}\left(1-w_{i_{0}}\right) \neq 0} \frac{1}{*\left(\mathscr{C}_{i_{0}}\right)} \cdot \frac{1}{\left.\mid \operatorname{cet}\left(P_{i_{0}}\left|A_{i_{0}}: P_{i_{0}}\right| A_{i_{0}}: w_{i_{0}}: 0\right) \cdot \mathbf{I n d}_{\mathscr{C}_{i_{0}}}^{G}\left(\left(\boldsymbol{\sigma}_{i_{0}}, 0\right)\right)(\alpha)\right\}} \\
& \cdot \operatorname{tr}\left\{\mathbf{c}\left(P_{i_{0}}\left|A_{i_{0}}: P_{i_{0}}\right| A_{i_{0}}: w_{i_{0}}: 0\right) \cdot \mathbf{I n d}_{\mathscr{C}_{i_{0}}}^{G}\left(\left(\boldsymbol{\sigma}_{i_{0}}, 0\right)\right)(\alpha)\right\}
\end{aligned}
$$

$\mathscr{C}_{0}$ being, as usual, the association class containing $\mathscr{C}_{i_{0}}$. The verification of this relation hinges on a combinatorial intermediary.

Write

$$
\left\{\begin{array}{l}
\amalg W\left(A_{i_{0}}\right) / \sim=\amalg \mathfrak{w}_{0}, \\
\mathfrak{w}_{0}=\amalg \mathfrak{w}_{i_{0}},
\end{array}\right.
$$

where $w_{i_{0}} \sim w_{j_{0}}$ iff

$$
\exists w_{j_{0} i_{0}} \in W\left(A_{j_{0}}, A_{i_{0}}\right) \quad \text { st } \quad w_{j_{0}}=w_{j_{0} i_{0}} w_{i_{0}} w_{j_{0} i_{0}}^{-1} .
$$


A generic $\mathfrak{w}_{0}$ can be regarded as an association class of conjugacy classes since each $\mathfrak{w}_{i_{0}}$ is an ordinary group-theoretic conjugacy class in $W\left(A_{i_{0}}\right)$.

Recast, the question thus becomes that of the equality of

$$
\sum_{\mathfrak{w}_{0}} \sum_{\mathfrak{w}_{t_{0}}} \sum_{w_{t_{0}} \in \mathfrak{w}_{t_{0}}: \operatorname{det}\left(1-w_{\iota_{0}}\right) \neq 0} c\left(w_{i_{0}}\right) \cdots
$$

and

$$
\sum_{\mathfrak{w}_{0}} \sum_{\mathfrak{w}_{t_{0}}} \sum_{w_{t_{0}} \in \mathfrak{w}_{t_{0}}: \operatorname{det}\left(1-w_{i_{0}}\right) \neq 0} \frac{1}{*\left(\mathscr{C}_{i_{0}}\right)} \cdots
$$

Fix a $\mathfrak{w}_{0}$ admitting a representaive $w_{i_{0}}$ with $\operatorname{det}\left(1-w_{i_{0}}\right) \neq 0$. From the functional equations, it is clear that if

$$
\left\{\begin{array}{l}
w_{i_{0}} \in \mathfrak{w}_{i_{0}} \subset \mathfrak{w}_{0} \\
w_{j_{0}} \in \mathfrak{w}_{j_{0}} \subset \mathfrak{w}_{0}
\end{array}\right.
$$

then

$$
\begin{aligned}
& \operatorname{tr}\left\{\mathbf{c}\left(P_{i_{0}}\left|A_{i_{0}}: P_{i_{0}}\right| A_{i_{0}}: w_{i_{0}}: 0\right) \cdot \mathbf{I n d}_{\mathscr{C}_{i_{0}}}^{G}\left(\left(\boldsymbol{\Theta}_{i_{0}}, 0\right)\right)(\alpha)\right\} \\
& \left.\quad=\operatorname{tr}\left\{\mathbf{c}\left(P_{j_{0}}\left|A_{j_{0}}: P_{j_{0}}\right| A_{j_{0}}: w_{j_{0}}: 0\right) \cdot \mathbf{I n d}_{\mathscr{C}_{0}}^{G}\left(\boldsymbol{\sigma}_{j_{0}}, 0\right)\right)(\alpha)\right\} .
\end{aligned}
$$

Of course, it is understood that here $\boldsymbol{\theta}_{i_{0}}$ and $\boldsymbol{\theta}_{j_{0}}$ are associate. This said, it will therefore be enough to show that

$$
\sum_{\mathfrak{w}_{\iota_{0}}} \sum_{w_{\imath_{0}} \in \mathfrak{w}_{l_{0}}} c\left(w_{i_{0}}\right)=\frac{\#\left(\mathfrak{w}_{0}\right)}{*\left(\mathscr{C}_{i_{0}}\right)} .
$$

For this purpose, put

$$
\Omega_{i_{0}}=\coprod_{w_{t_{0}} \in \mathfrak{w}_{i_{0}}}\left(1-w_{i_{0}}\right) \Xi_{i_{0}} .
$$

We have now:

(1) $\forall w_{j_{0} i_{0}}, w_{j_{0} i_{0}} \Omega_{i_{0}}=\Omega_{j_{0}}$.

[In fact,

$$
\begin{aligned}
w_{j_{0} i_{0}} \Omega_{i_{0}} & =\coprod w_{j_{0} i_{0}}\left(1-w_{i_{0}}\right) \Xi_{i_{0}} \\
& =\coprod w_{j_{0} i_{0}}\left(1-w_{i_{0}}\right) w_{j_{0} i_{0}}^{-1} \Xi_{j_{0}} \\
& =\coprod\left(1-w_{j_{0} i_{0}} w_{i_{0}} w_{j_{0} i_{0}}^{-1}\right) \Xi_{j_{0}} \\
& \left.=\coprod\left(1-w_{j_{0}}\right) \Xi_{j_{0}}=\Omega_{j_{0}} .\right]
\end{aligned}
$$

(2) $\Omega_{i_{0}} \cap \overline{\mathscr{C}}_{P_{t_{0}}}=\Omega_{i_{0}} \cap \mathscr{C}_{P_{t_{0}}}$. 
[In fact,

$$
\left.\forall \xi_{i_{0}} \in \Xi_{i_{0}}, \quad\left(1-w_{i_{0}}\right) \xi_{i_{0}} \not \not \lambda_{i_{0}} \quad\left(\lambda_{i_{0}} \in \Sigma_{P_{i_{0}}}^{0}\right) .\right]
$$

(3) $\Omega_{i_{0}} \subset\left(\check{\mathfrak{a}}_{i_{0}}\right)_{\text {reg. }}$

[In fact, given any $\omega_{i_{0}} \in \Omega_{i_{0}}$, we can find a $w_{j_{0} i_{0}}$ for which $w_{j_{0} i_{0}} \omega_{i_{0}} \in$ $\overline{\mathscr{C}}_{P_{j_{0}}}$ (cf. Lemma 2 on p. 67 of TES), hence

$$
\begin{gathered}
\left.w_{j_{0} i_{0}} \omega_{i_{0}} \in\left(\check{\mathfrak{a}}_{j_{0}}\right)_{\text {reg }} \Rightarrow \omega_{i_{0}} \in\left(\check{\mathfrak{a}}_{i_{0}}\right)_{\text {reg }} .\right] \\
\frac{\#\left(\Omega_{i_{0}}\right)}{\#\left(W\left(A_{i_{0}}\right)\right)}=\sum_{j_{0}} \#\left(\Omega_{j_{0}} \cap-\mathscr{C}_{P_{j_{0}}}\right) .
\end{gathered}
$$

[In fact, for each $j_{0}$ choose a $w_{j_{0} i_{0}} \in W\left(A_{j_{0}}, A_{i_{0}}\right)$-then, since $W\left(A_{i_{0}}\right)$ acts simply transitively on $\left(\check{\mathfrak{a}}_{i_{0}}\right)_{\text {reg }}$ with fundamental domain

$$
\bigcup_{j_{0}} w_{j_{0} i_{0}}^{-1}\left(-\mathscr{C}_{P_{j_{0}}}\right)
$$

the union

$$
\bigcup_{j_{0}} w_{j_{0} i_{0}}^{-1}\left(\Omega_{j_{0}} \cap-\mathscr{C}_{P_{j_{0}}}\right)
$$

is disjoint, as is the union

$$
\begin{gathered}
\left.\boldsymbol{\Omega}_{i_{0}}=\bigcup_{w_{t_{0}}} w_{i_{0}}\left(\bigcup_{j_{0}} w_{j_{0} i_{0}}^{-1}\left(\boldsymbol{\Omega}_{j_{0}} \cap-\mathscr{C}_{P_{J_{0}}}\right)\right) .\right] \\
\sum_{w_{i_{0}} \in \mathfrak{w}_{t_{0}}} c\left(w_{i_{0}}\right)=\frac{\#\left(\boldsymbol{\Omega}_{i_{0}} \cap-\mathscr{C}_{P_{i_{0}}}\right)}{\#\left(\boldsymbol{\Xi}_{i_{0}}\right)} .
\end{gathered}
$$

[In fact, by definition

$$
\left.c\left(w_{i_{0}}\right)=\frac{\#\left\{\xi_{i_{0}} \in \Xi_{i_{0}}:\left(1-w_{i_{0}}\right) \xi_{i_{0}} \in-\mathscr{C}_{P_{i_{0}}}\right\}}{\#\left(\Xi_{i_{0}}\right)} .\right]
$$

Combining the preceding assertions, we get

$$
\begin{aligned}
\sum_{\mathfrak{w}_{t_{0}}} & \sum_{w_{\iota_{0}} \in \mathfrak{w}_{\iota_{0}}} c\left(w_{i_{0}}\right) \\
& =\frac{1}{\#\left(\Xi_{i_{0}}\right)} \cdot \frac{\#\left(\Omega_{i_{0}}\right)}{\left.\#\left(A_{i_{0}}\right)\right)} \\
& =\frac{1}{\#\left(\Xi_{i_{0}}\right)} \cdot \frac{1}{\#\left(W\left(A_{i_{0}}\right)\right)} \cdot \#\left(\Xi_{i_{0}}\right) \cdot \frac{\#\left(W\left(A_{i_{0}}\right)\right)}{\#\left(W\left(A_{i_{0}}\right)_{t_{0}}\right)} \\
& =\frac{1}{\#\left(W\left(A_{i_{0}}\right) w_{\iota_{0}}\right)} .
\end{aligned}
$$


On the other hand,

$$
\#\left(\mathfrak{w}_{0}\right)=\frac{*\left(\mathscr{C}_{i_{0}}\right)}{\#\left(W\left(A_{i_{0}}\right)\right)} \cdot \frac{\#\left(W\left(A_{i_{0}}\right)\right)}{\#\left(W\left(A_{i_{0}}\right)_{w_{i_{0}}}\right)}
$$

or still

$$
\frac{\#\left(\mathfrak{w}_{0}\right)}{*\left(\mathscr{C}_{i_{0}}\right)}=\frac{1}{\#\left(W\left(A_{i_{0}}\right)_{w_{\iota_{0}}}\right)}
$$

It follows that

$$
\sum_{\mathfrak{w}_{i_{0}}} \sum_{w_{\iota_{0}} \in \mathfrak{w}_{i_{0}}} c\left(w_{i_{0}}\right)=\frac{\#\left(\mathfrak{w}_{0}\right)}{*\left(\mathscr{C}_{i_{0}}\right)},
$$

as desired.

Appendix. The purpose of this Appendix is to say a few words about Assumption c. Recall the statement:

Assumption c. For each $K$-type $\delta$, there exist positive constants $\varepsilon$, $M$, and $N$ with the following property:

$$
\begin{aligned}
& \forall i_{0}, \forall \boldsymbol{\Theta}_{i_{0}}, \forall \Lambda_{i_{0}} \in \sqrt{-1} \check{\mathfrak{a}}_{i_{0}}, \forall \lambda_{i_{0}} \in \check{\mathfrak{a}}_{i_{0}}, \forall w_{j_{0} i_{0}} \in W\left(A_{j_{0}}, A_{i_{0}}\right): \\
& \left\|\mathbf{c}\left(P_{j_{0}}\left|A_{j_{0}}: P_{i_{0}}\right| A_{i_{0}}: w_{j_{0} i_{0}}: \Lambda_{i_{0}}+\lambda_{i_{0}}\right)\right\|_{\text {OP }} \\
& \quad<M \cdot\left(1+\left\|\boldsymbol{\Theta}_{i_{0}}\right\|\right)^{N} \cdot\left(1+\left\|\Lambda_{i_{0}}\right\|\right)^{N}
\end{aligned}
$$

provided

$$
\left\|\lambda_{i_{0}}\right\|<\varepsilon \cdot\left(1+\left\|\boldsymbol{\theta}_{i_{0}}\right\|\right)^{-N} \cdot\left(1+\left\|\Lambda_{i_{0}}\right\|\right)^{-N} .
$$

We hardly need point out that this assumption has been of pivotal importance for our development of the theory. The requirement of polynomial growth in the parameters is totally compatible with what we know (and expect) about the behavior of the c-functions. Of course, the absence of estimates of the desired type is a serious gap in our understanding. Someday we hope to clear up this lacuna by a systematic application of "hard" function theory to Langland's approach of the meromorphic continuation in the rank-1 case but no serious steps in this direction have yet been taken. It should be noted, however, that it suffices to treat just the rank-1 case. Indeed, a given $w\left(=w_{j_{0} i_{0}}\right)$ can always be decomposed into a product of simple reflections, say $w=w_{1} \cdots w_{n}$, thus, by the functional equations,

$$
\|\mathbf{c}(w)\|_{\mathrm{OP}} \leq \prod\left\|\mathbf{c}\left(w_{i}\right)\right\|_{\mathrm{OP}}
$$

from which the assertion. 
In $\S 4$ of [2-(e)] we formulated the following

Main Conjecture. The operator $L_{G / \Gamma}^{\mathrm{dis}}(\alpha)$ is trace class for every $K$ finite $\alpha$ in $C_{c}^{\infty}(G)$.

We offered there two hypotheses, RH and GRH, and proved that either one of them implies MC. Actually, these hypotheses and the assumption at hand are closely related. In fact:

Proposition. Assumption c $\Rightarrow$ Main Conjecture.

Maintaining the notation of $\S 4$ of [2-(e)], inspection of the discussion therein shows that it is enough to verify that $\forall \mathfrak{E}, \forall \mathfrak{X} \in \mathfrak{E}$,

$$
-\left(X\left(P_{i_{0}}, A_{i_{0}}\right), \rho_{i_{0}}\right) \geq C_{\delta} \cdot\left(1+\left\|\Theta_{i_{0}}\right\|\right)^{-L_{\delta}} .
$$

To this end, let us admit:

Lemma. Under Assumption c, $\forall \mathfrak{E}, \forall \mathfrak{X} \in \mathfrak{E}$,

$$
\left\|X\left(P_{i_{0}}, A_{i_{0}}\right)\right\| \geq C_{\delta}^{0} \cdot\left(1+\left\|\mathscr{\sigma}_{i_{0}}\right\|\right)^{-L_{\delta}} .
$$

It is then an easy matter to pass to the

Proof of the Proposition. Write

$$
\rho_{i_{0}}=\sum_{1}^{l_{0}} c_{i_{0}} \lambda^{i_{0}} \quad\left(c_{i_{0}}>0\right) .
$$

Put (cf. [2-(b), p. 440])

$$
\|?\|_{\rho_{t_{0}}}=\sum_{1}^{l_{0}} c_{i_{0}} \cdot\left|\left(?, \lambda^{i_{0}}\right)\right| .
$$

Then $\|?\|_{\rho_{t_{0}}}$ is a norm on $\check{\mathfrak{a}}_{i_{0}}$, thus, by equivalence of norms, there exists a positive constant $C\left(\rho_{i_{0}}\right)$ such that

$$
\|?\| \leq C\left(\rho_{i_{0}}\right) \cdot\|?\|_{\rho_{\iota_{0}}} .
$$

Accordingly,

$$
\begin{aligned}
C_{\delta}^{0} \cdot\left(1+\left\|\boldsymbol{\sigma}_{i_{0}}\right\|\right)^{-L_{\delta}} & \leq\left\|X\left(P_{i_{0}}, A_{i_{0}}\right)\right\| \\
& \leq C\left(\rho_{i_{0}}\right) \cdot\left\|X\left(P_{i_{0}}, A_{i_{0}}\right)\right\|_{\rho_{i_{0}}} \\
& =C\left(\rho_{i_{0}}\right) \cdot \sum_{1}^{l_{0}} c_{i_{0}} \cdot\left|\left(X\left(P_{i_{0}}, A_{i_{0}}\right), \lambda^{i_{0}}\right)\right| \\
& =-C\left(\rho_{i_{0}}\right) \cdot \sum_{1}^{l_{0}} c_{i_{0}} \cdot\left(X\left(P_{i_{0}}, A_{i_{0}}\right), \lambda^{i_{0}}\right) \\
& =-C\left(\rho_{i_{0}}\right) \cdot\left(X\left(P_{i_{0}}, A_{i_{0}}\right), \rho_{i_{0}}\right),
\end{aligned}
$$


that is,

$$
-\left(X\left(P_{i_{0}}, A_{i_{0}}\right), \rho_{i_{0}}\right) \geq \frac{C_{\delta}^{0}}{C\left(\rho_{i_{0}}\right)} \cdot\left(1+\left\|\boldsymbol{\theta}_{i_{0}}\right\|\right)^{-L_{\delta}},
$$

as desired.

Turning now to the lemma, the point is that $X\left(P_{i_{0}}, A_{i_{0}}\right)$ must lie on a singular hyperplane of

$$
\mathbf{c}_{\text {cus }}\left(P_{j_{0}}\left|A_{j_{0}}: P_{i_{0}}\right| A_{i_{0}}: w_{j_{0} i_{0}}: ?\right)
$$

for some $j_{0}$ and $w_{j_{0} i_{0}}$. That this is so is contained in the theory of amalgamations, in particular the proof of the Main Lemma in TES (pp. 258-259). Consider the table:

\begin{tabular}{|c|c|c|c|c|}
\hline & $\mathfrak{X}_{i_{0}}$ & $\mathbf{I}_{i_{0}}^{\prime}$ & $\mathbf{I}_{i_{0}}^{\prime \prime}$ & $\mathbf{I}_{i_{0}}^{\prime \prime \prime}$ \\
\hline $\operatorname{Amal}(0)$ & $\varnothing$ & $\varnothing$ & $\{\varnothing\}$ & $\varnothing$ \\
\hline $\operatorname{Amal}(1)$ & Can Amal & $\varnothing$ & $*$ & $\varnothing$ \\
\hline $\operatorname{Amal}(2)$ & $*$ & $*^{\prime}$ & $*^{\prime \prime}$ & $*^{\prime \prime \prime}$ \\
\hline
\end{tabular}

\section{Here,}

* = all singular hyperplanes of Can Amal (TES, p. 254) which meet the tube over the negative chamber.

$*^{\prime}, *^{\prime \prime}, *^{\prime \prime \prime} \subset$ singular hyperplanes of some $\mathrm{c}_{\text {cus }}$.

What we want can therefore be read off from this compilation. That being, if our contention were false, then we could take in Assumption c

$$
\left\{\begin{array}{l}
\lambda_{i_{0}}=X\left(P_{i_{0}}, A_{i_{0}}\right) \\
\Lambda_{i_{0}}=0
\end{array}\right.
$$

to conclude that

$$
\left\|\mathbf{c}_{\text {cus }}\left(P_{j_{0}}\left|A_{j_{0}}: P_{j_{0}}\right| A_{j_{0}}: w_{j_{0} i_{0}}: X\left(P_{i_{0}}, A_{i_{0}}\right)\right)\right\|_{\mathrm{OP}}
$$

is finite, an absurdity.

\section{REFERENCES}

[1-(a)] J. Arthur, On a family of distributions obtained from Eisenstein series I, Amer. J. Math., 104 (1982), 1243-1288.

[1-(b)] _ On a family of distributions obtained from Eisenstein series II, Amer. J. Math., 104 (1982), 1289-1336. 
[2-(a)] M. S. Osborne, and G. Warner, The Selberg trace formula I, Crelle's J., 324 (1981), 1-113.

[2-(b)] _ The Selberg trace formula II, Pacific J. Math., 106 (1983), 307-496.

[2-(c)] _ The Selberg trace formula III, Memoirs Amer. Math. Soc., 283 (1983), 1-209.

[2-(d)] _ , The Selberg trace formula IV, SLN, 1024 (1983), 112-263.

[2-(e)] _ The Selberg trace formula V, Trans. Amer. Math. Soc., 286 (1984), 351-376.

[2-(f)] _ , The Selberg trace formula, VI, Amer. J. Math., 107 (1985), 1369-1437.

Received October 28, 1987. Research by both authors supported in part by the National Science Foundation.

UNIVERSITY OF WASHINGTON

SeAtTLE, WA 98195 


\section{PACIFIC JOURNAL OF MATHEMATICS EDITORS}

\author{
V. S. VARAdarajan \\ (Managing Editor) \\ University of California \\ Los Angeles, CA 90024-1555-05 \\ Herbert Clemens \\ University of Utah \\ Salt Lake City, UT 84112 \\ ThOMAS ENRIGHT \\ University of California, San Diego \\ La Jolla, CA 92093
}

R. FINN

Stanford University

Stanford, CA 94305

HeRmann FlaschKa

University of Arizona

Tucson, AZ 85721

VAUGHAN F. R. Jones

University of California

Berkeley, CA 94720

SteVen KercKhofF

Stanford University

Stanford, CA 94305

\section{ROBION KIRBY}

University of California

Berkeley, CA 94720

C. C. MOORE

University of California

Berkeley, CA 94720

HAROLD STARK

University of California, San Diego

La Jolla, CA 92093

\section{ASSOCIATE EDITORS}
R. Arens
E. F. BeCKenbaCH
B. H. NEUMANN
F. Wolf
K. YoshidA (1906-1982)

\section{SUPPORTING INSTITUTIONS}

UNIVERSITY OF ARIZONA

UNIVERSITY OF BRITISH COLUMBIA

CALIFORNIA INSTITUTE OF TECHNOLOGY

UNIVERSITY OF CALIFORNIA

MONTANA STATE UNIVERSITY

UNIVERSITY OF NEVADA, RENO

NEW MEXICO STATE UNIVERSITY OREGON STATE UNIVERSITY

\author{
UNIVERSITY OF OREGON \\ UNIVERSITY OF SOUTHERN CALIFORNIA \\ STANFORD UNIVERSITY \\ UNIVERSITY OF HAWAII \\ UNIVERSITY OF TOKYO \\ UNIVERSITY OF UTAH \\ WASHINGTON STATE UNIVERSITY \\ UNIVERSITY OF WASHINGTON
}

The Supporting Institutions listed above contribute to the cost of publication of this Journal, but they are not owners or publishers and have no responsibility for its content or policies.

Mathematical papers intended for publication in the Pacific Journal of Mathematics should be in typed form or offset-reproduced (not dittoed), double spaced with large margins. Please do not use built up fractions in the text of the manuscript. However, you may use them in the displayed equations. Underline Greek letters in red, German in green, and script in blue. The first paragraph must be capable of being used separately as a synopsis of the entire paper. In particular it should contain no bibliographic references. Please propose a heading for the odd numbered pages of less than 35 characters. Manuscripts, in triplicate, may be sent to any one of the editors. Please classify according to the scheme of Math. Reviews, Index to Vol. 39. Supply name and address of author to whom proofs should be sent. All other communications should be addressed to the managing editor, or Elaine Barth, University of California, Los Angeles, California 90024-1555-05.

There are page-charges associated with articles appearing in the Pacific Journal of Mathematics. These charges are expected to be paid by the author's University, Government Agency or Company. If the author or authors do not have access to such Institutional support these charges are waived. Single authors will receive 50 free reprints; joint authors will receive a total of 100 free reprints. Additional copies may be obtained at cost in multiples of 50 .

The Pacific Journal of Mathematics is issued monthly as of January 1966. Regular subscription rate: $\$ 190.00$ a year (5 Vols., 10 issues). Special rate: $\$ 95.00$ a year to individual members of supporting institutions.

Subscriptions, orders for numbers issued in the last three calendar years, and changes of address should be sent to Pacific Journal of Mathematics, P.O. Box 969, Carmel Valley, CA 93924, U.S.A. Old back numbers obtainable from Kraus Periodicals Co., Route 100, Millwood, NY 10546.

The Pacific Journal of Mathematics at P.O. Box 969, Carmel Valley, CA 93924 (ISSN 0030-8730) publishes 5 volumes per year. Application to mail at Second-class postage rates is pending at Carmel Valley, California, and additional mailing offices. Postmaster: send address changes to Pacific Journal of Mathematics, P.O. Box 969, Carmel Valley, CA 93924.

PUBLISHED BY PACIFIC JOURNAL OF MATHEMATICS, A NON-PROFIT CORPORATION Copyright (C) 1989 by Pacific Journal of Mathematics 


\section{Pacific Journal of Mathematics \\ Vol. 140, No. $2 \quad$ October, 1989}

Edoardo Ballico, Spanned and ample vector bundles with low Chern numbers

Marcy Mason Barge, Richard Swanson and Russell Bruce Walker,

Conjugacy class structure of smooth hyperbolic sectors . ........... 217

Jeffrey Stephen Fox, Adeles and the spectrum of compact nilmanifolds . . ..233

Robert D. Little, Homotopy complex projective spaces with divisible

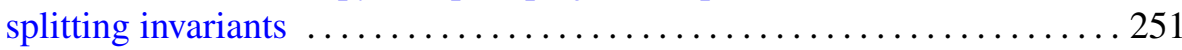

M. Scott Osborne and Garth William Warner, Jr., The Selberg trace formula. VII. Application of the truncation process to the continuous

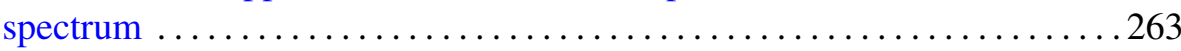

John R. Stembridge, On the eigenvalues of representations of reflection

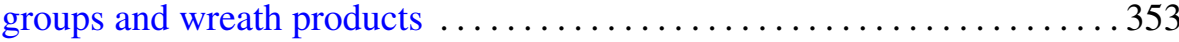

Ibrahim Salama, Corrections to: "Topological entropy and recurrence of

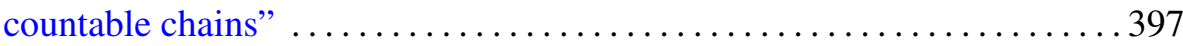

Robert Greene and Hung-Hsi Wu, Addendum to: "Lipschitz convergence of Riemannian manifolds" ............................... 398

Kayoko Shikishima-Tsuji, Correction to: "Galois theory of differential fields of positive characteristic" 